\title{
Modular sheaves on hyperkähler varieties
}

\author{
Kieran G. O'Grady \\ Dedicato a Titti
}

\begin{abstract}
A torsion-free sheaf on a hyperkähler manifold $X$ is modular if its discriminant satisfies a certain condition; for example, this is the case if it is a multiple of $c_{2}(X)$. The definition is tailor-made for torsion-free sheaves on a polarized hyperkähler variety $(X, h)$ which deform to all small deformations of $(X, h)$. For hyperkähler varieties of type $K 3^{[2]}$, we prove an existence and uniqueness result for slope-stable vector bundles with certain ranks, $c_{1}$ and $c_{2}$. As a consequence, we get the uniqueness up to isomorphism of the tautological quotient rank 4 vector bundle on the variety of lines on a generic cubic 4dimensional hypersurface, and on the Debarre-Voisin variety associated with a generic element of $\bigwedge^{3} \mathbb{C}^{10}$. The last result implies that the period map from the moduli space of Debarre-Voisin varieties to the relevant period space is birational.
\end{abstract}

\section{Introduction}

1.1. Background and motivation. The beautiful properties of vector bundles on $K 3$ surfaces play a prominent role in algebraic geometry. Since $K 3$ surfaces are the 2-dimensional hyperkähler (HK) compact manifolds, one is tempted to explore the world of vector bundles on higherdimensional HK manifolds. In the present paper, we give way to this temptation. Our proposal is to focus attention on vector bundles, or more generally (coherent) torsion-free sheaves, whose Chern character satisfies a certain condition; see Definition 1.1. We call such sheaves modular. The definition is tailor-made for torsion-free sheaves on a polarized HK manifold $(X, h)$ which deform to all small deformations of $(X, h)$. With this hypothesis, we may deform $(X, h)$ to a Lagrangian $\left(X_{0}, h_{0}, \pi\right)$, where $\pi: X_{0} \rightarrow \mathbb{P}^{n}$ is a Lagrangian fibration, study stable sheaves $\mathscr{F}$ on $X_{0}$ by studying the restriction of $\mathscr{F}$ to a generic fiber of $\pi$ (an abelian variety of dimension $n$ ) and then deduce properties of the initial moduli space of sheaves on $(X, h)$. This strategy has been implemented in the case of $K 3$ surfaces; see [O'G97]. A successful implementation in higher dimensions requires an extension of the known results regarding the variation of $h$ slope-stability of a sheaf. More precisely, one needs to know that, given a class $\chi \in H(X ; \mathbb{Q})$, there exists a decomposition of the ample cone into open chambers such that, given a sheaf $\mathscr{F}$ with $\operatorname{ch}(\mathscr{F})=\chi$ and an open chamber $\mathscr{C}$, the sheaf $\mathscr{F}$ is $h$ slope-stable either for all $h \in \mathscr{C}$ or for no such $h$. Modular sheaves on HK manifolds are exactly the sheaves for which one can prove that such a

Received 20 September 2020, accepted in final form 21 April 2021.

2020 Mathematics Subject Classification 14J42, 14J60.

Keywords: hyperkähler varieties, stable sheaves.

This journal is (C) Foundation Compositio Mathematica 2022. This article is distributed with Open Access under the terms of the Creative Commons Attribution Non-Commercial License, which permits non-commercial reuse, distribution, and reproduction in any medium, provided that the original work is properly cited. For commercial re-use, please contact the Foundation Compositio Mathematica.

The author was partially supported by PRIN 2015 . 


\section{K. G. O'GRADY}

decomposition of the ample cone exists. Another remarkable consequence of our definition is the following. Let $\pi: X \rightarrow \mathbb{P}^{n}$ be a Lagrangian fibration, and let $\mathscr{F}$ be a modular vector bundle on $X$ whose restriction to a generic fiber of $\pi$ is slope-stable; then the restriction of $\mathscr{F}$ to a generic fiber is a semi-homogeneous vector bundle (according to Mukai's definition; see [Muk78]), and hence it has no infinitesimal deformations fixing the determinant. This shows that, in the case of modular sheaves, the strategy outlined above in higher dimensions resembles that which has been implemented in the case of $K 3$ surfaces (notice that a slope-stable vector bundle on an elliptic curve has by default no infinitesimal deformations fixing the determinant, while this certainly does not hold for vector bundles on abelian surfaces - it holds exactly for semi-homogeneous ones).

1.2. Modular sheaves. Let $\mathscr{F}$ be a rank $r$ torsion-free sheaf on a manifold $X$. The discriminant $\Delta(\mathscr{F}) \in H_{\mathbb{Z}}^{2,2}(X)$ is defined to be

$$
\Delta(\mathscr{F}):=2 r c_{2}(\mathscr{F})-(r-1) c_{1}(\mathscr{F})^{2}=-2 r \operatorname{ch}_{2}(\mathscr{F})+\operatorname{ch}_{1}(\mathscr{F})^{2} .
$$

Below is our key definition.

Definition 1.1. Let $X$ be a HK manifold of dimension $2 n$, and let $q_{X}$ be its BeauvilleBogomolov-Fujiki (BBF) bilinear symmetric form. A torsion-free sheaf $\mathscr{F}$ on $X$ is modular if there exists a $d(\mathscr{F}) \in \mathbb{Q}$ such that

$$
\int_{X} \Delta(\mathscr{F}) \smile \alpha^{2 n-2}=d(\mathscr{F}) \cdot(2 n-3) ! ! \cdot q_{X}(\alpha)^{n-1}
$$

for all $\alpha \in H^{2}(X)$.

Remark 1.2. Let $X$ be a HK variety of dimension $2 n$. Let $D(X) \subset H(X)$ be the image of the map Sym $H^{2}(X) \rightarrow H(X)$ defined by the cup product. Let $D^{i}(X):=D(X) \cap H^{i}(X)$. The pairing $D^{i}(X) \times D^{4 n-i}(X) \rightarrow \mathbb{C}$ defined by the intersection product is nondegenerate [Ver96a, Bog96, Bea07], hence there is a splitting $H(X)=D(X) \oplus D(X)^{\perp}$, where orthogonality is with respect to the intersection pairing. Now let $\mathscr{F}$ be a torsion-free sheaf on $X$. Then $\mathscr{F}$ is modular if and only if the orthogonal projection of $\Delta(\mathscr{F})$ onto $D^{4}(X)$ is a multiple of the class $q_{X}^{\vee}$ dual to $q_{X}$. Moreover, if $\Delta(\mathscr{F})$ is a multiple of $c_{2}(X)$, then $\mathscr{F}$ is modular by Fujiki's formula; see [Fuj87, Remark 4.12].

Remark 1.3. Let $X$ be a HK manifold of type $K 3^{[2]}$. Then $H(X)=D(X)$ (notation as in Remark 1.2). It follows that a vector bundle $\mathscr{F}$ on $X$ is modular if and only if $\Delta(\mathscr{F})$ is a multiple of $c_{2}(X)$. It follows [Ver96b] that if $\mathscr{F}$ is a modular vector bundle, slope-stable for a polarization $h$, then $\operatorname{End}^{0}(\mathscr{F})$ is hyperholomorphic on $(X, h)$, where $\operatorname{End}^{0}(\mathscr{F})$ is the vector bundle of traceless endomorphisms of $\mathscr{E}$. More generally, on an arbitrary HK polarized variety $(X, h)$, there should be a relation between the property of being modular and that of being hyperholomorphic.

1.3. Main results. Let $\mathscr{E}$ be a modular torsion-free sheaf on a HK manifold $X$ of type $K 3^{[2]}$. A simple argument, see Proposition 2.3, shows that $r(\mathscr{E})$ divides the square of a generator of the ideal $\left\{q_{X}\left(c_{1}(\mathscr{E}), \alpha\right) \mid \alpha \in H^{2}(X ; \mathbb{Z})\right\}$. We give an existence and uniqueness statement for slope-stable vector bundles $\mathscr{E}$ such that $r(\mathscr{E})$ equals the square of a generator of the ideal defined above, and moreover

$$
\Delta(\mathscr{E})=\frac{r(\mathscr{E})(r(\mathscr{E})-1)}{12} c_{2}(X)
$$




\section{MODULAR SHEAVES ON HYPERKÄHLER VARIETIES}

Before formulating our results, we recall the description of the irreducible components of the moduli space of polarized HK manifolds of type $K 3^{[2]}$. Let $(X, h)$ be one such polarized HK manifold (we emphasize that the ample class $h \in H_{\mathbb{Z}}^{1,1}(X)$ is primitive). Then either

$$
q\left(h, H^{2}(X ; \mathbb{Z})\right)=\mathbb{Z}, \quad q(h)=e>0, \quad e \equiv 0 \quad(\bmod 2),
$$

or

$$
q\left(h, H^{2}(X ; \mathbb{Z})\right)=2 \mathbb{Z}, \quad q(h)=e>0, \quad e \equiv 6 \quad(\bmod 8) .
$$

Conversely, if $e$ is a positive integer which is even (respectively, congruent to 6 modulo 8), there exists an $(X, h)$ such that (1.3.1) (respectively, (1.3.2)) holds. Let $\mathscr{K}_{e}^{1}$ be the moduli space of polarized HK manifolds $(X, h)$ of type $K 3^{[2]}$ such that $(1.3 .1)$ holds, and let $\mathscr{K}_{e}^{2}$ be the moduli space of polarized HK manifolds $(X, h)$ of type $K 3^{[2]}$ such that (1.3.2) holds.

Theorem 1.4. Let $i \in\{1,2\}$, and let $r_{0}$ and $e$ be positive integers such that $r_{0} \equiv i(\bmod 2)$ and

$$
e \equiv\left\{\begin{array}{lll}
4 r_{0}-10 \quad\left(\bmod 8 r_{0}\right) & \text { if } r_{0} \equiv 0 & (\bmod 4), \\
\frac{1}{2}\left(r_{0}-5\right) \quad\left(\bmod 2 r_{0}\right) & \text { if } r_{0} \equiv 1 & (\bmod 4) \\
-10 \quad\left(\bmod 8 r_{0}\right) & \text { if } r_{0} \equiv 2 & (\bmod 4), \\
-\frac{1}{2}\left(r_{0}+5\right) \quad\left(\bmod 2 r_{0}\right) & \text { if } r_{0} \equiv 3 & (\bmod 4) .
\end{array}\right.
$$

Suppose that $[(X, h)] \in \mathscr{K}_{e}^{i}$ is a generic point. Then, up to isomorphism, there exists one and only one $h$ slope-stable vector bundle $\mathscr{E}$ on $X$ such that

$$
r(\mathscr{E})=r_{0}^{2}, \quad c_{1}(\mathscr{E})=\frac{r_{0}}{i} h, \quad \Delta(\mathscr{E})=\frac{r(\mathscr{E})(r(\mathscr{E})-1)}{12} c_{2}(X) .
$$

Moreover, $H^{p}\left(X, \operatorname{End}^{0}(\mathscr{E})\right)=0$ for all $p$.

Remark 1.5. Let $[(X, h)] \in \mathscr{K}_{6}^{2}$ be generic. Then $(X, h)$ is isomorphic to the variety of lines $F(Y)$ on a generic cubic hypersurface $Y \subset \mathbb{P}^{5}$ polarized by the Plücker embedding, and the vector bundle $\mathscr{E}$ of Theorem 1.4 with $r_{0}=2$ is isomorphic to the restriction of the tautological quotient vector bundle on $\operatorname{Gr}\left(2, \mathbb{C}^{6}\right)$. Similarly, let $[(X, h)] \in \mathscr{K}_{22}^{2}$ be generic. Then $(X, h)$ is isomorphic to the Debarre-Voisin variety associated with a generic $\sigma \in \wedge^{3} V_{10}^{\vee}$, where $V_{10}$ is a 10-dimensional complex vector space, and

$$
X_{\sigma}:=\left\{[W] \in \operatorname{Gr}\left(6, V_{10}\right)|\sigma|_{W}=0\right\} .
$$

The vector bundle $\mathscr{E}$ of Theorem 1.4 with $r_{0}=2$ is isomorphic to the restriction to $X_{\sigma}$ of the tautological quotient vector bundle on $\operatorname{Gr}\left(6, V_{10}\right)$. These results are proved in Section 8 .

Remark 1.6. We would like the congruence relations in (1.3.3) to be forced upon us by adding to the hypotheses on $r(\mathscr{E}), c_{1}(\mathscr{E}), \Delta(\mathscr{E})$ the extra hypothesis that $\chi\left(X, \operatorname{End}^{0}(\mathscr{E})\right)=0$. Our computations give this for some values of $r_{0}$, but we do not have complete results.

The result below replaces the genericity hypothesis in Theorem 1.4 with a cohomological one.

Corollary 1.7. Let $i \in\{1,2\}$, and let $r_{0}$ and $e$ be positive integers such that $r_{0} \equiv i(\bmod 2)$ and (1.3.3) holds. Let $[(X, h)] \in \mathscr{K}_{e}^{i}$. Suppose that $\mathscr{E}$ is an $h$ slope-stable vector bundle on $X$ such that (1.3.4) holds and $H^{2}\left(X, \operatorname{End}^{0}(\mathscr{E})\right)=0$. If $\mathscr{G}$ is an $h$ slope-stable vector bundle on $X$ and $\operatorname{ch}_{k}(\mathscr{G})=\operatorname{ch}_{k}(\mathscr{E})$ for $k \in\{0,1,2\}$, then $\mathscr{G}$ is isomorphic to $\mathscr{E}$.

There is an interesting consequence of Theorem 1.4 involving Debarre-Voisin (DV) varieties. There is a geometric invariant theory moduli space $\mathscr{M}_{\mathrm{DV}}:=\mathbb{P}\left(\bigwedge^{3} V_{10}^{\vee}\right) / / \mathrm{SL}\left(V_{10}\right)$ of DV varieties; 


\section{K. G. O'GRADY}

see [DHOV20]. In [DV10] it was proved that the moduli map $\mathscr{M}_{\mathrm{DV}} \rightarrow-\rightarrow \mathscr{K}_{22}^{2}$ has finite nonzero degree.

Theorem 1.8. The moduli map $\mathscr{M}_{\mathrm{DV}} \rightarrow-\rightarrow \mathscr{K}_{22}^{2}$ is birational.

Theorem 1.4 is also relevant to the study of degenerate DV varieties which was carried out in [DHOV20]. We say a few words about this in Subsection 8.3.

1.4. Outline of the paper. In Section 2, we give a few examples of modular sheaves, and we make the connection with semi-homogeneous vector bundles. In particular, we give strong restrictions on the possible ranks of modular sheaves, under some hypotheses.

Section 3 contains the results that extend to modular sheaves the known results on the variation of slope-stability for sheaves on surfaces. In particular, we show that one can extend the results that hold for sheaves on surfaces which are fibered over a curve to HK manifolds with a Lagrangian fibration.

In Section 4, we prove properties of slope-stable modular vector bundles on HK manifolds $X$ of type $K 3^{[2]}$ with a Lagrangian fibration $X \rightarrow \mathbb{P}^{2}$. We make certain hypotheses; in particular, we assume that $r(\mathscr{E})$ equals the square of a generator of the ideal $\left\{q_{X}\left(c_{1}(\mathscr{E}), \alpha\right) \mid \alpha \in H^{2}(X ; \mathbb{Z})\right\}$. We show that the restriction of a slope-stable modular vector bundle on $X$ to a generic Lagrangian fiber is slope-stable and that if $\mathscr{F}$ is another such vector bundle, then the restrictions of $\mathscr{E}$ and $\mathscr{F}$ to a generic Lagrangian fiber are isomorphic.

Section 5 discusses a construction which associates with a vector bundle $\mathscr{F}$ on a $K 3$ surface $S$ two torsion-free sheaves $\mathscr{F}[n]^{ \pm}$on $S^{[n]}$ whose fibers over a reduced scheme $\left\{x_{1}, \ldots, x_{n}\right\}$ are the tensor product $\mathscr{F}\left(x_{1}\right) \otimes \cdots \otimes \mathscr{F}\left(x_{n}\right)$ of the fibers of $\mathscr{F}$ at the points $x_{1}, \ldots, x_{n}$ this is a generalization of a construction which was given in [DHOV20]. We prove that if $\chi\left(S, \mathscr{F}^{\vee} \otimes \mathscr{F}\right)=2$, then $\mathscr{F}[2]^{ \pm}$is a modular vector bundle, and we compute its Chern character. As proved in Section 7, this construction gives (by deformation) the existence result of Theorem 1.4.

In Section 6 , we let $S \rightarrow \mathbb{P}^{1}$ be an elliptic $K 3$ surface with Picard number 2 . Then $S^{[2]}$ has an associated Lagrangian fibration $\pi: S^{[2]} \rightarrow\left(\mathbb{P}^{1}\right)^{(2)} \cong \mathbb{P}^{2}$. We prove that if $\mathscr{F}$ is a slope-stable rigid vector bundle on $S$, then the vector bundle $\mathscr{F}[2]^{ \pm}$on $S^{[2]}$ has good properties. In particular, we show that it extends to any small deformation of $S^{[2]}$ which keeps $c_{1}\left(\mathscr{F}[2]^{ \pm}\right)$of type $(1,1)$ and that the restriction to any fiber of the Lagrangian fibration $\pi$ is simple.

Section 7 contains the proof of Theorem 1.4 (and of Corollary 1.7). The basic idea is as follows. Let $\mathscr{X} \rightarrow T_{e}^{i}$ be a complete family of polarized HK manifolds of type $K 3^{[2]}$ whose moduli belong to $\mathscr{K}_{e}^{i}$. By results of Gieseker and Maruyama, there is a relative moduli scheme $f: \mathscr{M}_{e}\left(r_{0}\right) \rightarrow T_{e}^{i}$ whose fiber over $t \in T_{e}^{i}$ is the moduli space of slope-stable vector bundles on $\left(X_{t}, h_{t}\right)$ with the given rank, $c_{1}$ and $c_{2}$. The map $f: \mathscr{M}_{e}\left(r_{0}\right) \rightarrow T_{e}^{i}$ is of finite type by a result of Maruyama. Let $\mathscr{M}_{e}^{*}\left(r_{0}\right) \subset \mathscr{M}_{e}\left(r_{0}\right)$ be the (open) subset parametrizing vector bundles whose $\mathrm{ch}_{0}, \mathrm{ch}_{1}, \mathrm{ch}_{2}$ are given by the formulae in Theorem 1.4. Because of the good properties of the vector bundles $\mathscr{F}[2]^{ \pm}$, the image $f\left(\mathscr{M}_{e}^{*}\left(r_{0}\right)\right)$ contains a dense open (in the Zariski topology) subset of $T_{e}^{i}$. On the other hand, the results of Sections 4 and 6 allow us to prove that, up to isomorphism, there is a unique slope-stable vector bundle with the relevant $\mathrm{ch}_{0}, \mathrm{ch}_{1}, \mathrm{ch}_{2}$ on a generic $\mathrm{HK}$ manifold parametrized by a Lagrangian Noether-Lefschetz locus with large discriminant. By the density of the union of Noether-Lefschetz divisors (with large discriminant), we conclude that $f$ has degree 1 . 


\section{MODUlAR SHEAVES ON HYPERKÄHLER VARIETIES}

In Section 8, we prove Theorem 1.8. Once we have Theorem 1.4, the main point is to show that the tautological quotient vector bundle on a generic DV variety is slope-stable.

In the appendix, we discuss properties of semi-homogeneous vector bundles on abelian varieties and of Lagrangian Noether-Lefschetz divisors on moduli spaces of polarized HK manifolds of type $K 3^{[2]}$.

\subsection{Conventions}

- Algebraic variety is synonymous with complex quasi-projective (not necessarily irreducible) variety.

- Let $X$ be a smooth complex quasi-projective variety and $\mathscr{F}$ a coherent sheaf on $X$. We only consider topological Chern classes $c_{i}(\mathscr{F}) \in H^{2 i}(X(\mathbb{C}) ; \mathbb{Z})$.

- Let $X$ be a HK manifold of dimension $2 n$. We let $q_{X}$, or simply $q$, be the BBF symmetric bilinear form of $X$, and we denote $q_{X}(\alpha, \alpha)$ by $q_{X}(\alpha)$. We let $c_{X}$ be the normalized Fujiki constant of $X$, that is, the positive rational number such that for all $\alpha \in H^{2}(X)$, we have

$$
\int_{X} \alpha^{2 n}=c_{X} \cdot(2 n-1) ! ! \cdot q_{X}(\alpha)^{n} .
$$

A hyperkähler (HK) variety is a projective compact HK manifold.

- Let $\mathscr{F}$ be a torsion-free sheaf on a polarized projective variety $(X, h)$. A subsheaf $\mathscr{E} \subset \mathscr{F}$ is slope-destabilizing if $0<r(\mathscr{E})<r(\mathscr{F})$ and $\mu_{h}(\mathscr{E}) \geqslant \mu_{h}(\mathscr{F})$, where $r(\mathscr{E})$ and $r(\mathscr{F})$ are the ranks of $\mathscr{E}$ and $\mathscr{F}$, respectively, and $\mu_{h}(\mathscr{E})$ and $\mu_{h}(\mathscr{F})$ are the $h$-slopes of $\mathscr{E}$ and $\mathscr{F}$, respectively. If $\mu_{h}(\mathscr{E})>\mu_{h}(\mathscr{F})$, then $\mathscr{E} \subset \mathscr{F}$ is slope-desemistabilizing. We use similar terminology for exact sequences $0 \rightarrow \mathscr{E} \rightarrow \mathscr{F} \rightarrow \mathscr{G} \rightarrow 0$.

- A torsion-free sheaf on $(X, h)$ is strictly $h$ slope-semistable if it is $h$ slope-semistable but not $h$ slope-stable.

- Abusing notation, we say that a smooth projective variety $X$ is an abelian variety if it is isomorphic to the variety underlying an abelian variety $A$. In other words, $X$ is a torsor of $A$.

\section{Modular sheaves}

2.1. First examples. Remark 1.2 leads to the following examples of modular vector bundles:

(1) The tangent bundle $\Theta_{X}$ is modular.

(2) Let $V_{6}$ be a 6 -dimensional complex vector space, and let $X \subset \operatorname{Gr}\left(2, V_{6}\right)$ be the variety of lines contained in a smooth cubic hypersurface in $\mathbb{P}\left(V_{6}\right)$. Let $h \in H_{\mathbb{Z}}^{1,1}(X)$ be the Plücker polarization. Then $X$ is a HK variety of type $K 3^{[2]}$; see [BD85]. Let $\mathscr{Q}$ be the restriction to $X$ of the tautological rank 4 quotient vector bundle on $\operatorname{Gr}\left(2, V_{6}\right)$. We claim that

$$
\operatorname{ch}_{0}(\mathscr{Q})=4, \quad \operatorname{ch}_{1}(\mathscr{Q})=h, \quad \operatorname{ch}_{2}(\mathscr{Q})=\frac{1}{8}\left(h^{2}-c_{2}(X)\right) .
$$

The first two equations are obvious; the last equation can be obtained as follows. Let $\mathscr{U}$ be the restriction to $X$ of the tautological subbundle on $\operatorname{Gr}\left(2, V_{6}\right)$. The normal bundle sequence

$$
0 \longrightarrow \Theta_{X} \longrightarrow \Theta_{\operatorname{Gr}\left(2, V_{6}\right) \mid X} \longrightarrow \operatorname{Sym}^{3} \mathscr{U}^{\vee} \longrightarrow 0
$$

gives that $\operatorname{ch}_{2}(\mathscr{U})=-\left(h^{2}-c_{2}(X)\right) / 8$. Since $\operatorname{ch}(\mathscr{Q})=6-\operatorname{ch}(\mathscr{U})$, this gives the last equation in (2.1.1). Thus $\Delta(\mathscr{Q})=c_{2}(X)$, and hence $\mathscr{Q}$ is modular. 


\section{K. G. O'GRADY}

(3) Let $X \subset \operatorname{Gr}\left(6, V_{10}\right)$ be a smooth DV variety, and let $h \in H_{\mathbb{Z}}^{1,1}(X)$ be the Plücker polarization; see [DV10]. Then $X$ is a HK variety of type $K 3^{[2]}$. Let $\mathscr{Q}$ be the restriction to $X$ of the tautological rank 4 quotient vector bundle on $\operatorname{Gr}\left(6, V_{10}\right)$. Then

$$
\operatorname{ch}_{0}(\mathscr{Q})=4, \quad \operatorname{ch}_{1}(\mathscr{Q})=h, \quad \operatorname{ch}_{2}(\mathscr{Q})=\frac{1}{8}\left(h^{2}-c_{2}(X)\right) .
$$

The above equations follow from the computations in [DV10, Proof of Lemma 4.5, p. 83]; see Lemma 8.1. Thus $\Delta(\mathscr{Q})=c_{2}(X)$, and hence $\mathscr{Q}$ is modular.

Remark 2.1. Let $X$ be a HK variety, and let $\mathscr{E}$ and $\mathscr{F}$ be modular sheaves on $X$. Then $\mathscr{E} \oplus \mathscr{F}$ is not modular in general. On the other hand, $\mathscr{E} \otimes \mathscr{F}$ is modular, at least if $\mathscr{E}$ and $\mathscr{F}$ are locally free.

Remark 2.2. Let $X$ be a HK manifold of dimension $2 n$, and let $\mathscr{F}$ be a torsion-free modular sheaf on $X$. Then

$$
\int_{X} \Delta(\mathscr{F}) \smile \alpha_{1} \smile \ldots \smile \alpha_{2 n-2}=d(\mathscr{F}) \cdot \widetilde{\sum} q_{X}\left(\alpha_{i_{1}}, \alpha_{i_{2}}\right) \cdot \ldots \cdot q_{X}\left(\alpha_{i_{2 n-3}}, \alpha_{i_{2 n-2}}\right)
$$

for all $\alpha_{1}, \ldots, \alpha_{2 n} \in H^{2}(X)$, where $\tilde{\Sigma}$ means that in the summation we avoid repeating addends which are formally equal (that is, are equal modulo reordering of the factors $q_{X}(\cdot, \cdot)$ and switching the entries in $\left.q_{X}(\cdot, \cdot)\right)$.

2.2. Restrictions on the rank. Below is the result that was mentioned in Section 1.3.

Proposition 2.3. Let $X$ be a $H K$ fourfold of type $K 3^{[2]}$ or $\mathrm{Kum}_{2}$. Let $\mathscr{F}$ be a modular torsionfree sheaf on $X$. Let $m$ be a generator of the ideal

$$
\left\{q_{X}\left(c_{1}(\mathscr{F}), \alpha\right) \mid \alpha \in H^{2}(X ; \mathbb{Z})\right\} .
$$

Then $r(\mathscr{F})$ divides $m^{2}$ if $X$ is of type $K 3^{[2]}$, and it divides $3 m^{2}$ if $X$ is of type $\mathrm{Kum}_{2}$.

Proof. As is easily checked, there exists an $\alpha \in H^{2}(X ; \mathbb{Z})$ such that $q_{X}\left(c_{1}(\mathscr{F}), \alpha\right)=m$ and $q_{X}(\alpha)=0$. Let $r:=r(\mathscr{F})$. Since $q_{X}(\alpha)=0$, equation (1.2.2) gives that

$$
\begin{aligned}
2 r \int_{X} c_{2}(\mathscr{F}) \smile \alpha^{2} & =(r-1) \int_{X} c_{1}(\mathscr{F})^{2} \smile \alpha^{2} \\
& =2(r-1) c_{X} \cdot q_{X}\left(c_{1}(\mathscr{F}), \alpha\right)^{2}=2(r-1) c_{X} \cdot m^{2} .
\end{aligned}
$$

The result follows because $c_{X}=1$ if $X$ is of type $K 3^{[2]}$ and $c_{X}=3$ if $X$ is of type $\mathrm{Kum}_{2}$.

2.3. Modular sheaves on Lagrangian fibrations. We recall that a Lagrangian fibration $\pi: X \rightarrow \mathbb{P}^{n}$ on a HK manifold $X$ of dimension $2 n$ is a surjective map with connected fibers whose smooth fibers are abelian varieties.

Remark 2.4. For $t \in \mathbb{P}^{n}$, we let $X_{t}:=\pi^{-1}(t)$ be the schematic fiber over $t$. If $X_{t}$ is smooth, the image of the restriction map $H^{2}(X ; \mathbb{Z}) \rightarrow H^{2}\left(X_{t} ; \mathbb{Z}\right)$ has rank 1 and is generated by an ample class $\theta_{t} \in H_{\mathbb{Z}}^{1,1}\left(X_{t}\right)$; see [Wie16]. If $\mathscr{F}$ is a sheaf on $X_{t}$, slope-(semi)stability of $\mathscr{F}$ will always mean $\theta_{t}$ slope-(semi)stability.

If $\pi: X \rightarrow \mathbb{P}^{n}$ is a Lagrangian fibration, we let

$$
f:=c_{1}\left(\pi^{*} \mathscr{O}_{\mathbb{P} n}(1)\right) \in H_{\mathbb{Z}}^{1,1}(X) .
$$

As is well known, $q_{X}(f)=0$. 


\section{MODUlAR SHEAVES ON HYPERKÄHLER VARIETIES}

Lemma 2.5. Let $\pi: X \rightarrow \mathbb{P}^{n}$ be a Lagrangian fibration of a HK manifold of dimension $2 n$. Suppose that $\mathscr{F}$ is a modular torsion-free sheaf on $X$. Let $t \in \mathbb{P}^{n}$ be a general point, and let $\mathscr{F}_{t}:=\mathscr{F}_{\mid X_{t}}$ be the restriction of $\mathscr{F}$ to $X_{t}$. Then

$$
\int_{X_{t}} \Delta\left(\mathscr{F}_{t}\right) \smile \theta_{t}^{n-2}=0 .
$$

Proof. There exists an $\omega \in H^{2}(X ; \mathbb{Z})$ such that $\theta_{t}=\omega_{\mid X_{t}}$. Since $t \in \mathbb{P}^{n}$ is a generic point, we have $\Delta\left(\mathscr{F}_{t}\right)=\Delta(\mathscr{F})_{\mid X_{t}}$. Moreover, $f^{n}$ is the Poincaré dual of $X_{t}$. Hence

$$
\int_{X_{t}} \Delta(\mathscr{F} t) \smile \theta_{t}^{n-2}=\int_{X} \Delta(\mathscr{F}) \smile \omega^{n-2} \smile f^{n} .
$$

The integral on the right vanishes by Remark 2.2 and the equality $q_{X}(f)=0$.

Example 2.6. Let $S$ be a $K 3$ surface, and let $V$ be a vector bundle on $S$. Let $\mathscr{Z} \subset S \times S^{[n]}$ be the tautological subscheme, and let $p: \mathscr{Z} \rightarrow S$ and $q: \mathscr{Z} \rightarrow S^{[n]}$ be the projection maps. The locally free sheaf $q_{*}\left(p^{*} V\right)$ is known as a tautological sheaf on $S^{[n]}$. In general, such a sheaf is not modular. In fact, suppose that $S$ is elliptic, with elliptic fibration $S \rightarrow \mathbb{P}^{1}$. The composition $S^{[n]} \rightarrow S^{(n)} \rightarrow\left(\mathbb{P}^{1}\right)^{(n)} \cong \mathbb{P}^{n}$ is a Lagrangian fibration with generic fiber $X_{t}=C_{1} \times \cdots \times C_{n}$, where $C_{1}, \ldots, C_{n}$ are distinct generic fibers of the elliptic fibration $S \rightarrow \mathbb{P}^{1}$. If the restriction of $V$ to the fibers of $S \rightarrow \mathbb{P}^{1}$ has nonzero degree, then equality (2.3.2) does not hold for $\mathscr{F}:=q_{*}\left(p^{*} V\right)$, and hence $q_{*}\left(p^{*} V\right)$ is not modular.

Proposition 2.7. Let $\pi: X \rightarrow \mathbb{P}^{n}$ be a Lagrangian fibration of a $H K$ manifold of dimension $2 n$. Let $\mathscr{F}$ be a modular torsion-free sheaf on $X$. Suppose that $t \in \mathbb{P}^{n}$ is a regular value of $\pi$, that $\mathscr{F}$ is locally free in a neighborhood of $X_{t}$ and that $\mathscr{F}_{t}$ is slope-stable. Then $\mathscr{F}_{t}$ is a semi-homogeneous vector bundle.

Proof. This follows from Lemma 2.5 and Proposition A.2.

The result below shows that, under suitable hypotheses, a much stronger version of Proposition 2.3 holds.

Corollary 2.8. Let $X$ be a $H K$ variety of type $K 3^{[n]}, \mathrm{Kum}_{n}$ or OG6. Let $\mathscr{F}$ be a modular torsion-free sheaf on $X$. Suppose that $t \in \mathbb{P}^{n}$ is a regular value of $\pi$, that $\mathscr{F}$ is locally free in a neighborhood of $X_{t}$ and that $\mathscr{F}_{t}$ is slope-stable. Then there exist positive integers $r_{0}, d$, with $d$ dividing $c_{X}$, such that $r(\mathscr{F})=r_{0}^{n} / d$.

Proof. If $X$ is of type $K 3^{[n]}$, then $c_{X}=1$ and $\theta_{t}$ is (up to multiplication by a nonzero scalar) a principal polarization; see [Wie16]. If $X$ is of type $\mathrm{Kum}_{n}$ or OG6, then $c_{X}=n+1$ and $\theta_{t}$ is (up to multiplication by a nonzero scalar) a polarization with elementary divisors $\left(1, \ldots, 1, d_{1}, d_{2}\right)$, where $d_{1} \cdot d_{2}$ divides $n+1$; see [Wie18] for $\mathrm{Kum}_{n}$ and [MR21] for OG6. Hence the result follows from Propositions 2.7 and A.3.

\section{Variation of stability for modular sheaves}

3.1. Main results. Let $X$ be an irreducible smooth projective variety. If the ample cone $\operatorname{Amp}(X)$ has rank greater than 1 (and hence $\operatorname{dim} X \geqslant 2$ ), the slope-stability of a sheaf $\mathscr{F}$ depends on the choice of an ample ray. If $X$ is a surface, there is a locally finite decomposition $\operatorname{Amp}(X)_{\mathbb{R}}$ into chambers defined by rational walls such that slope-stability is the same for ample 


\section{K. G. O'GRADY}

classes belonging to the same open chamber. One important feature is that the walls depend only on the Chern character of $\mathscr{F}$.

If $\operatorname{dim} X \geqslant 3$, the picture is more intricate in general; see, for example, [GRT19].

In the present section, we show that if $X$ is a $\mathrm{HK}$ variety and $\mathscr{F}$ is a modular sheaf, then there is a decomposition of $\operatorname{Amp}(X)_{\mathbb{R}}$ as if $X$ were a surface.

Definition 3.1. Let $a$ be a positive real number. An $a$-wall of $\operatorname{Amp}(X)_{\mathbb{R}}$ is the intersection $\lambda^{\perp} \cap \operatorname{Amp}(X)_{\mathbb{R}}$, where $\lambda \in H_{\mathbb{Z}}^{1,1}(X),-a \leqslant q_{X}(\lambda)<0$ and orthogonality is with respect to the BBF quadratic form $q_{X}$.

As is well known, the set of $a$-walls is locally finite; in particular, the union of all the $a$-walls is closed in $\operatorname{Amp}(X)_{\mathbb{R}}$.

DEFINITION 3.2. An open a-chamber is a connected component of the complement $\left(\operatorname{in} \operatorname{Amp}(X)_{\mathbb{R}}\right)$ of the union of all the $a$-walls.

Definition 3.3. Let $X$ be a HK manifold, and let $\mathscr{F}$ be a modular torsion-free sheaf on $X$. Then

$$
a(\mathscr{F}):=\frac{r(\mathscr{F})^{2} \cdot d(\mathscr{F})}{4 c_{X}},
$$

where $d(\mathscr{F})$ is as in Definition 1.1.

Below is the first main result.

Proposition 3.4. Let $X$ be a $H K$ variety of dimension $2 n$, and let $\mathscr{F}$ be a torsion-free modular sheaf on $X$. Then the following hold:

(i) Suppose that $h$ is an ample divisor class on $X$ which belongs to an open a( $\mathscr{F})$-chamber. If $\mathscr{F}$ is strictly $h$ slope-semistable, there exists an exact sequence of torsion-free nonzero sheaves

$$
0 \longrightarrow \mathscr{E} \longrightarrow \mathscr{F} \longrightarrow \mathscr{G} \longrightarrow 0
$$

such that $r(\mathscr{F}) c_{1}(\mathscr{E})-r(\mathscr{E}) c_{1}(\mathscr{F})=0$.

(ii) Suppose that $h_{0}$ and $h_{1}$ are ample divisor classes on $X$ belonging to the same open $a(\mathscr{F})$ chamber. Then $\mathscr{F}$ is $h_{0}$ slope-stable if and only if it is $h_{1}$ slope-stable.

Proposition 3.4 is proved in Subsection 3.3.

The next result is about slope-stable sheaves on HK varieties which carry a Lagrangian fibration.

DeFinition 3.5. Let $X$ be a HK variety equipped with a Lagrangian fibration $\pi: X \rightarrow \mathbb{P}^{n}$, and let $f:=\pi^{*} c_{1}\left(\mathscr{O}_{\mathbb{P}^{n}}(1)\right)$. Let $a$ be a positive integer. An ample divisor class $h$ on $X$ is a-suitable if the following holds. Let $\lambda \in H_{\mathbb{Z}}^{1,1}(X)$ be a class such that $-a \leqslant q_{X}(\lambda)<0$; then either $q_{X}(\lambda, h)$ and $q_{X}(\lambda, f)$ have the same sign, or they are both zero.

Notice that the notion of $a$-suitable depends on the chosen Lagrangian fibration.

Proposition 3.6. Let $\pi: X \rightarrow \mathbb{P}^{n}$ be a Lagrangian fibration of a $H K$ variety of dimension $2 n$. Let $\mathscr{F}$ be a torsion-free modular sheaf on $X$ such that sing $\mathscr{F}$ does not dominate $\mathbb{P}^{n}$. Let $h$ be an ample divisor class on $X$ which is a $\mathscr{F})$-suitable. Then the following hold:

(i) If the restriction of $\mathscr{F}$ to a generic fiber of $\pi$ is slope-stable, then $\mathscr{F}$ is $h$ slope-stable.

(ii) If $\mathscr{F}$ is $h$ slope-stable, then the restriction of $\mathscr{F}$ to the generic fiber of $\pi$ is slope-semistable.

Proposition 3.6 is proved in Section 3.5. 


\section{MODUlAR SHEAVES ON HYPERKÄHLER VARIETIES}

3.2. Change of slope-stability and strictly semistable sheaves. Suppose that $\mathscr{E}$ and $\mathscr{F}$ are sheaves on an irreducible smooth variety $X$. We let

$$
\lambda_{\mathscr{E}, \mathscr{F}}:=\left(r(\mathscr{F}) c_{1}(\mathscr{E})-r(\mathscr{E}) c_{1}(\mathscr{F})\right) \in H^{2}(X ; \mathbb{Z}) .
$$

Lemma 3.7. Let $(X, h)$ be a polarized $H K$ variety, and let $\mathscr{E}$ and $\mathscr{F}$ be nonzero torsion-free sheaves on $X$. Then

(i) $\mu_{h}(\mathscr{E})>\mu_{h}(\mathscr{F})$ if and only if $q_{X}\left(\lambda_{\mathscr{E}, \mathscr{F}}, h\right)>0$;

(ii) $\mu_{h}(\mathscr{E})=\mu_{h}(\mathscr{F})$ if and only if $q_{X}\left(\lambda_{\mathscr{E}, \mathscr{F}}, h\right)=0$.

Proof. Let $2 n$ be the dimension of $X$. We have $\mu_{h}(\mathscr{E})>\mu_{h}(\mathscr{F})$ if and only if $\int_{X} \lambda_{\mathscr{E}, \mathscr{F}} \smile h^{2 n-1}>$ 0 , and by Fujiki's formula, this holds if and only if

$$
c_{X} \cdot(2 n-1) ! ! \cdot q_{X}\left(\lambda_{\mathscr{E}, \mathscr{F}}, h\right) \cdot q_{X}(h)^{n-1}>0 .
$$

Item (i) follows because $c_{X}>0$ and $q_{X}(h)>0$.

We have $\mu_{h}(\mathscr{E})=\mu_{h}(\mathscr{F})$ if and only if $\int_{X} \lambda_{\mathscr{E}, \mathscr{F}} \smile h^{2 n-1}=0$, and hence item (ii) follows again by Fujiki's formula.

Proposition 3.8. Let $X$ be a $H K$ variety, and let $h_{0}$ and $h_{1}$ be ample divisor classes on $X$. Suppose that $\mathscr{F}$ is a torsion-free sheaf on $X$ which is $h_{0}$ slope-stable and not $h_{1}$ slope-stable. Then there exists an $h \in\left(\mathbb{Q}_{+} h_{0}+\mathbb{Q}_{+} h_{1}\right)$ such that $\mathscr{F}$ is strictly $h$ slope-semistable, that is, $\mathscr{F}$ is $h$ slope-semistable but not $h$ slope-stable.

Proof. Lemma 3.7 allows us to reproduce the proof of the analogous statement valid for surfaces (see $[$ HL10]). Let $\mathrm{S} \subset([0,1] \cap \mathbb{Q})$ be the set of $s$ for which there exists a subsheaf $\mathscr{E} \subset \mathscr{F}$ with $0<r(\mathscr{E})<r(\mathscr{F})$ such that

$$
q_{X}\left(\lambda_{\mathscr{E}, \mathscr{F}},(1-s) h_{0}+s h_{1}\right)=0 .
$$

Then $\mathrm{S}$ is nonempty and finite. In fact, by hypothesis, there exists an $h_{1}$ destabilizing subsheaf $\mathscr{E} \subset \mathscr{F}$. Thus $0<r(\mathscr{E})<r(\mathscr{F})$, and $q_{X}\left(\lambda_{\mathscr{E}, \mathscr{F}}, h_{1}\right) \geqslant 0$ by Lemma 3.7. On the other hand, by the same lemma, $q_{X}\left(\lambda_{\mathscr{E}, \mathscr{F}}, h_{0}\right)<0$ because $\mathscr{F}$ is $h_{0}$ slope-stable. It follows that there exists an $s \in[0,1] \cap \mathbb{Q}$ such that (3.2.2) holds; that is, $\mathrm{S}$ is not empty.

In order to prove that $\mathrm{S}$ is finite, assume that (3.2.2) holds. Since $\mathscr{F}$ is $h_{0}$ slope-stable, $q_{X}\left(\lambda_{\mathscr{E}, \mathscr{F}}, h_{0}\right)<0$. By the linearity of $q_{X}\left(\lambda_{\mathscr{E}, \mathscr{F}}, \cdot\right)$, we get that $q_{X}\left(\lambda_{\mathscr{E}, \mathscr{F}}, h_{1}\right) \geqslant 0$. By Lemma 3.7, it follows that

$$
\mu_{h_{1}}(\mathscr{E}) \geqslant \mu_{h_{1}}(\mathscr{F})
$$

The set of subsheaves $\mathscr{E} \subset \mathscr{F}$ such that (3.2.3) holds is bounded (see [HL10, Lemma 1.7.9]); that is, up to isomorphism, each such sheaf belongs to a finite set of families, each parametrized by an irreducible quasi-projective variety. It follows that $\mathrm{S}$ is finite because the values of $q_{X}\left(\lambda_{\mathscr{E}, \mathscr{F}}, h_{i}\right)$ for $i \in\{0,1\}$ are constant for sheaves $\mathscr{E}$ parametrized by an irreducible variety.

Since $\mathrm{S}$ is finite, there is a minimum $s$, call it $s_{\min }$, such that (3.2.2) holds for some subsheaf $\mathscr{E} \subset \mathscr{F}$ with $0<r(\mathscr{E})<r(\mathscr{F})$. Clearly $\mathscr{F}$ is strictly $\left(h_{0}+s_{\min } h_{1}\right)$ slope-semistable.

\subsection{Strictly semistable modular sheaves}

LEMMA 3.9. Let

$$
0 \longrightarrow \mathscr{E} \longrightarrow \mathscr{F} \longrightarrow \mathscr{G} \longrightarrow 0
$$

be an exact sequence of sheaves on a smooth variety. Then

$$
r(\mathscr{F}) \cdot r(\mathscr{G}) \Delta(\mathscr{E})+r(\mathscr{F}) \cdot r(\mathscr{E}) \Delta(\mathscr{G})=r(\mathscr{E}) \cdot r(\mathscr{G}) \Delta(\mathscr{F})+\lambda_{\mathscr{E}, \mathscr{F}}^{2}
$$




\section{K. G. O'Grady}

Proof. This follows from the additivity of the Chern character and the second equality in (1.2.1).

Proposition 3.10. Let $(X, h)$ be a polarized $H K$ variety of dimension $2 n$. Let $\mathscr{F}$ be a torsion-free modular strictly $h$ slope-semistable sheaf on $X$, and let

$$
0 \longrightarrow \mathscr{E} \longrightarrow \mathscr{F} \longrightarrow \mathscr{G} \longrightarrow 0
$$

be an exact sequence of nonzero torsion-free sheaves which is $h$ slope-destabilizing; that is, $\mu_{h}(\mathscr{E})=\mu_{h}(\mathscr{F})$. Then

$$
-a(\mathscr{F}) \leqslant q_{X}\left(\lambda_{\mathscr{E}, \mathscr{F}}\right) \leqslant 0
$$

Moreover, $q_{X}\left(\lambda_{\mathscr{E}, \mathscr{F}}\right)=0$ only if $\lambda_{\mathscr{E}, \mathscr{F}}=0$.

Proof. Since the exact sequence in (3.3.3) is destabilizing, $q_{X}\left(\lambda_{\mathscr{E}, \mathscr{F}}, h\right)=0$ by Lemma 3.7. Since the $\mathrm{BBF}$ form on $\mathrm{NS}(X)$ has signature $(1, \rho(X)-1)$, it follows that $q_{X}\left(\lambda_{\mathscr{E}, \mathscr{F}}\right) \leqslant 0$ with equality only if $\lambda_{\mathscr{E}, \mathscr{F}}=0$. (Recall that $q_{X}(h)>0$ because $h$ is ample.)

We are left with proving the second inequality in (3.3.4). Hence we assume that $q_{X}\left(\lambda_{\mathscr{E}, \mathscr{F}}\right)<0$. Cupping both sides of the equality in (3.3.2) by $h^{2 n-2}$ and integrating, we get (here we use the hypothesis that $\mathscr{F}$ is modular)

$$
\begin{aligned}
& \int_{X} r(\mathscr{F}) \cdot r(\mathscr{G}) \Delta(\mathscr{E}) \smile h^{2 n-2}+\int_{X} r(\mathscr{F}) \cdot r(\mathscr{E}) \Delta(\mathscr{G}) \smile h^{2 n-2} \\
& \quad=r(\mathscr{E}) \cdot r(\mathscr{G}) \cdot d(\mathscr{F}) \cdot(2 n-3) ! ! q_{X}(h)^{n-1}+c_{X} \cdot q_{X}\left(\lambda_{\mathscr{E}, \mathscr{F}}\right) \cdot(2 n-3) ! ! q_{X}(h)^{n-1} .
\end{aligned}
$$

By hypothesis, $\mu_{h}(\mathscr{E})=\mu_{h}(\mathscr{F})=\mu_{h}(\mathscr{G})$. Since $\mathscr{F}$ is $h$ slope-semistable, it follows that $\mathscr{E}$ and $\mathscr{G}$ are $h$ slope-semistable torsion-free sheaves. Thus

$$
\int_{X} \Delta(\mathscr{E}) \smile h^{2 n-2} \geqslant 0, \quad \int_{X} \Delta(\mathscr{G}) \smile h^{2 n-2} \geqslant 0
$$

by Bogomolov's inequality, and hence (3.3.5) gives

$$
-r(\mathscr{E}) \cdot r(\mathscr{G}) \cdot d(\mathscr{F}) \leqslant c_{X} \cdot q_{X}\left(\lambda_{\mathscr{E}, \mathscr{F}}\right) .
$$

Dividing by $c_{X}$ (which is strictly positive), we see that the second inequality in (3.3.4) follows from (3.3.6) and the inequality $r(\mathscr{E}) \cdot r(\mathscr{G}) \leqslant r(\mathscr{F})^{2} / 4$.

3.4 Proof of Proposition 3.4. Item (i) follows from Proposition 3.10. We prove item (ii). By symmetry, it suffices to show that if $\mathscr{F}$ is $h_{0}$ slope-stable, then it is $h_{1}$ slope-stable. Suppose that $\mathscr{F}$ is not $h_{1}$ slope-stable. By Proposition 3.8, there exists an $h \in\left(\mathbb{Q}_{+} h_{0}+\mathbb{Q}_{+} h_{1}\right)$ such that $\mathscr{F}$ is strictly $h$ slope-semistable. Hence there exists an $h$ destabilizing exact sequence

$$
0 \longrightarrow \mathscr{E} \longrightarrow \mathscr{F} \longrightarrow \mathscr{G} \longrightarrow 0
$$

of nonzero torsion-free sheaves. Since $h_{0}$ and $h_{1}$ belong to the same open $a(\mathscr{F})$-chamber, $h$ also belongs to the same open $a(\mathscr{F})$-chamber. Thus, by Proposition 3.10 , we get that $\lambda_{\mathscr{E}, \mathscr{F}}=0$. It follows that $\mathscr{F}$ is not $h_{0}$ slope-stable, and that gives a contradiction.

\subsection{Stability of modular sheaves on a Lagrangian HK variety}

LEMma 3.11. Let $X$ be a $H K$ variety of dimension $2 n$ equipped with a Lagrangian fibration $\pi: X \rightarrow \mathbb{P}^{n}$, and let $f:=c_{1}\left(\pi^{*} \mathscr{O}_{\mathbb{P}^{n}}(1)\right)$. Let $\mathscr{F}$ be a torsion-free sheaf on $X$, and let $\mathscr{E} \subset \mathscr{F}$ be a subsheaf with $0<r(\mathscr{E})<r(\mathscr{F})$. Then the following hold: 


\section{MODULAR SHEAVES ON HYPERKÄHLER VARIETIES}

(i) If, for generic $t \in \mathbb{P}^{n}$, the restriction $\mathscr{F}_{t}:=\mathscr{F}_{X_{t}}$ is slope-stable, then

$$
q_{X}\left(\lambda_{\mathscr{E}, \mathscr{F}}, f\right)<0 .
$$

(ii) If, for generic $t \in \mathbb{P}^{n}$, the subsheaf $\mathscr{E}_{t}:=\mathscr{E}_{\left.\right|_{X}} \subset \mathscr{F}_{t}$ is slope-desemistabilizing, then

$$
q_{X}\left(\lambda_{\mathscr{E}, \mathscr{F}}, f\right)>0 .
$$

Proof. Let $h_{t}:=h_{\mid X_{t}}$. We have

$$
\begin{aligned}
\int_{X_{t}} \lambda_{\mathscr{E}_{t}, \mathscr{F}_{t}} \smile h_{t}^{n-1} & =\int_{X} \lambda_{\mathscr{E}, \mathscr{F}} \smile h^{n-1} \smile f^{n} \\
& =n ! c_{X} \cdot q_{X}(h, f)^{n-1} \cdot q_{X}\left(\lambda_{\mathscr{E}, \mathscr{F}}, f\right) .
\end{aligned}
$$

In fact, the first equality holds because $f^{n}$ is the Poincaré dual of $X_{t}$, and the second equality holds by Fujiki's formula and the fact that $q_{X}(f)=0$. Items (i) and (ii) follow because $c_{X}$ and $q_{X}\left(\mathscr{O}_{X}(h), f\right)$ are strictly positive.

Proof of Proposition 3.6. We prove item (i). Suppose that $\mathscr{F}$ is not $h$ slope-stable. Let $\mathrm{S} \subset$ $([0,1] \cap \mathbb{Q})$ be the set of $s$ for which there exists a subsheaf $\mathscr{E} \subset \mathscr{F}$ with $0<r(\mathscr{E})<r(\mathscr{F})$ such that

$$
q_{X}\left(\lambda_{\mathscr{E}, \mathscr{F}},(1-s) h+s f\right)=0 .
$$

Let us show that $\mathrm{S}$ is nonempty and finite. Since $\mathscr{F}$ is not $h$ slope-stable, by Lemma 3.7 there exists a subsheaf $\mathscr{E} \subset \mathscr{F}$ with $0<r(\mathscr{E})<r(\mathscr{F})$ such that $q_{X}\left(\lambda_{\mathscr{E}, \mathscr{F}}, h\right) \geqslant 0$. On the other hand, by Lemma 3.11, the inequality in (3.5.1) holds. It follows that $\mathrm{S}$ is not empty. The argument in the proof of Proposition 3.8 showing that the analogous $\mathrm{S}$ is finite also applies in the present case, and hence $\mathrm{S}$ is finite.

Let $s_{\min }$ be the minimum element of S. Clearly $\mathscr{F}$ is strictly $h+s_{\min } f$ slope-semistable. Let $\mathscr{E} \subset \mathscr{F}$ be a subsheaf with $0<r(\mathscr{E})<r(\mathscr{F})$ which is $h+s_{\min } f$ destabilizing, that is, $q_{X}\left(\lambda_{\mathscr{E}, \mathscr{F}}, h+s_{\min } f\right)=0$. Then $-a(\mathscr{F}) \leqslant q_{X}\left(\lambda_{\mathscr{E}, \mathscr{F}}\right) \leqslant 0$ by Proposition 3.10 . On the other hand, $q_{X}\left(\lambda_{\mathscr{E}, \mathscr{F}}, f\right)<0$ by Lemma 3.11 , and hence $q_{X}\left(\lambda_{\mathscr{E}, \mathscr{F}}, h\right)<0$ by our hypothesis on $h$. This contradicts the equality $q_{X}\left(\lambda_{\mathscr{E}, \mathscr{F}}, h+s_{\min } f\right)=0$.

Next, we prove item (ii). Suppose that the restriction $\mathscr{F}_{\mid X_{t}}$ is $h_{t}$ slope-unstable for generic $t \in \mathbb{P}^{n}$. As before, let $\mathrm{S} \subset([0,1] \cap \mathbb{Q})$ be the subset of $s$ such that there exists a subsheaf $\mathscr{E} \subset \mathscr{F}$ with rank $0<r(\mathscr{E})<r(\mathscr{F})$ for which (3.5.4) holds. We claim that $\mathrm{S}$ is not empty and that it has a minimum (NB: it does not have a maximum).

In fact, since $\mathscr{F} X_{t}$ is $h_{t}$ slope-unstable for generic $t \in \mathbb{P}^{n}$, there exists a subsheaf $\mathscr{E} \subset \mathscr{F}$ with $0<r(\mathscr{E})<r(\mathscr{F})$ such that $\mathscr{E}_{t} \subset \mathscr{F}_{t}$ is $h_{t}$ slope-desemistabilizing for generic $t \in \mathbb{P}^{n}$. By Lemma 3.11, we have $q_{X}\left(\lambda_{\mathscr{E}, \mathscr{F}}, f\right)>0$. On the other hand, $q_{X}\left(\lambda_{\mathscr{E}, \mathscr{F}}, h\right)<0$ because $\mathscr{F}$ is $h$ slope-stable. It follows that $\mathrm{S}$ is not empty.

It remains to show that $\mathrm{S}$ has a minimum. Suppose that (3.5.4) holds. Since $q_{X}\left(\lambda_{\mathscr{E}, \mathscr{F}}, h\right)<0$ (because $\mathscr{F}$ is $h$ slope-stable), we get that $q_{X}\left(\lambda_{\mathscr{E}, \mathscr{F}}, f\right)>0$. Hence the sheaves $\mathscr{E} \subset \mathscr{F}$ with $0<r(\mathscr{E})<r(\mathscr{F})$ such that (3.5.4) holds for some $s \in[0,1] \cap \mathbb{Q}$ are exactly those such that $\mathscr{E}_{X_{t}} \subset \mathscr{F}_{X_{X}}$ is an $h_{t}$ slope-desemistabilizing sheaf of $\mathscr{F}_{X_{X}}$ for the generic $t \in \mathbb{P}^{n}$.

Let $\tilde{X}:=X \times \mathbb{P}^{n} \mathbb{C}\left(\mathbb{P}^{n}\right)$ be the abelian variety over $\mathbb{C}\left(\mathbb{P}^{n}\right)$ obtained from $X$ by base change. We let $\tilde{h}$ be the ample divisor on $\tilde{X}$ determined by $h$. A subsheaf $\mathscr{E} \subset \mathscr{F}$ on $X$ determines a subsheaf $\widetilde{\mathscr{E}} \subset \widetilde{\mathscr{F}}$ on $\widetilde{X}$.

Then $\mu_{\widetilde{h}}(\widetilde{\mathscr{E}})>\mu_{\widetilde{h}}(\widetilde{\mathscr{F}})$, that is, $\widetilde{\mathscr{E}}$ is $\widetilde{h}$ desemistabilizing for $\widetilde{\mathscr{F}}$, if and only if $\mathscr{E}_{X_{t}} \subset \mathscr{F}_{\left.\right|_{X_{t}}}$ is an $h_{t}$ slope-desemistabilizing sheaf of $\left.\mathscr{F}\right|_{X_{t}}$ for the generic $t \in \mathbb{P}^{n}$. The set of $\widetilde{h}$ desemistabilizing 


\section{K. G. O'Grady}

subsheaves $\mathscr{A} \subset \widetilde{\mathscr{F}}$ is bounded. Given such a subsheaf, there exists a unique maximal subsheaf $\mathscr{E} \subset \mathscr{F}$ such that $\widetilde{\mathscr{E}}=\mathscr{A}$. The set $\mathrm{S}^{0}$ of $s \in([0,1] \cap \mathbb{Q})$ such that (3.5.4) holds for such a maximal subsheaf is finite (and nonempty) by boundedness. Hence there is a minimum element $s_{\min }^{0}$ of $\mathrm{S}^{0}$. All other subsheaves $\mathscr{E} \subset \mathscr{F}$ (with $0<r(\mathscr{E})<r(\mathscr{F})$ ) such that $\widetilde{E} \subset \widetilde{F}$ is an $\tilde{h}$ desemistabilizing subsheaf are contained in a maximal subsheaf $\overline{\mathscr{E}}$, and the quotient $\overline{\mathscr{E}} / \mathscr{E}$ is supported on vertical divisors (that is, divisors whose image under $\pi$ is a proper subset of $\mathbb{P}^{n}$ ). It follows that $s_{\min }^{0}$ is also the minimum element of $\mathrm{S}$.

The sheaf $\mathscr{F}$ is strictly $\left(\left(1-s_{\min }\right) h+s_{\min } f\right)$ slope-semistable by the minimality of $s_{\text {min }}$. Let $\mathscr{E} \subset \mathscr{F}$ be a subsheaf with $0<r(\mathscr{E})<r(\mathscr{F})$ such that $q_{X}\left(\lambda_{\mathscr{E}, \mathscr{F}},\left(1-s_{\text {min }}\right) h+s_{\text {min }} f\right)=0$. By Proposition 3.10, either $-a(\mathscr{F}) \leqslant q_{X}\left(\lambda_{\mathscr{E}, \mathscr{F}}\right)<0$, or $\lambda_{\mathscr{E}, \mathscr{F}}=0$. The latter does not hold because $q_{X}\left(\lambda_{\mathscr{E}, \mathscr{F}}, h\right)<0$ (since $\mathscr{F}$ is $h$ slope-stable). Hence $-a(\mathscr{F}) \leqslant q_{X}\left(\lambda_{\mathscr{E}, \mathscr{F}}\right)<0$, and thus $q_{X}\left(\lambda_{\mathscr{E}, \mathscr{F}}, f\right)<0$ by our hypothesis on $h$. This contradicts the equality $q_{X}\left(\lambda_{\mathscr{E}, \mathscr{F}},\left(1-s_{\min }\right) h+\right.$ $\left.s_{\min } f\right)=0$.

\section{Stable vector bundles on Lagrangian HK varieties}

4.1. Main result. Before stating the main result, we recall that $\mathscr{K}_{e}^{i}$ is the moduli space of polarized HK varieties $(X, h)$ of type $K 3^{[2]}$ such that $q(h)=e$ and $h$ has divisibility $i$ (see (1.3.1) and (1.3.2)). Recall that $i=1$ if $e \neq \equiv 6(\bmod 8)$ and $i \in\{1,2\}$ if $e \equiv 6(\bmod 8)$.

The Noether-Lefschetz divisor $\mathscr{N}_{d}^{i}(e) \subset \mathscr{K}_{e}^{i}$ parametrizes the $(X, h)$ such that there exists a saturated rank 2 sublattice $\langle h, f\rangle \subset H_{\mathbb{Z}}^{1,1}(X)$, where $f$ is isotropic and $q(h, f)=d$; see Definition B.1. Assume that $d>10(e+1)$, that $e \nmid 2 d$ and that $d$ is even if $i=2$. By Proposition B.2, the divisor $\mathscr{N}_{d}^{i}(e)$ is of pure codimension 1 (in particular, nonempty), and there exists an open dense subset $\mathscr{N}_{d}^{i}(e)^{0} \subset \mathscr{N}_{d}^{i}(e)$ such that the following holds for $[(X, h)] \in \mathscr{N}_{d}^{i}(e)^{0}$ : there exists one and only one Lagrangian fibration $\pi: X \rightarrow \mathbb{P}^{2}$ (modulo automorphisms of $\mathbb{P}^{2}$ ) such that, letting $f:=\pi^{*} c_{1}\left(\mathscr{O}_{\mathbb{P}^{2}}(1)\right)$, the lattice $\langle h, f\rangle$ is as above. Below is the main result of the present section.

Proposition 4.1. Let $a_{0}$ and $d$ be positive integers, and let $i \in\{1,2\}$. Suppose that $e \nmid 2 d$, that $d$ is even if $i=2$ and that

$$
d>\max \left\{\frac{1}{2} a_{0}(e+1), 10(e+1)\right\} .
$$

If $[(X, h)] \in \mathscr{N}_{e}^{i}(d)^{0}$ is generic, the following hold:

(i) Let $\mathscr{E}$ be an $h$ slope-stable vector bundle on $X$ such that

(a) $a(\mathscr{E}) \leqslant a_{0}$, where $a(\mathscr{E})$ is as in Definition 3.3 ;

(b) there exists an integer $m$ such that $r(\mathscr{E})=(m i)^{2}, c_{1}(\mathscr{E})=m h$ and $\operatorname{gcd}\{m i, d / i\}=1$.

Then the restriction of $\mathscr{E}$ to a generic fiber of the associated Lagrangian fibration $\pi: X \rightarrow \mathbb{P}^{2}$ is slope-stable.

(ii) If $\mathscr{F}$ and $\mathscr{G}$ are $h$ slope-stable vector bundles on $X$ such that items (a) and (b) hold for $\mathscr{E}=\mathscr{F}$ and $\mathscr{E}=\mathscr{G}$, then for generic $z \in \mathbb{P}^{2}$, the restrictions of $\mathscr{F}$ and $\mathscr{G}$ to $\pi^{-1}(z)$ are isomorphic.

Remark 4.2. Regarding item (b) of Proposition 4.1: according to Proposition 2.3, we always have $r(\mathscr{E}) \mid(m i)^{2}$, hence the equality is an extremal case. 


\section{MODUlAR SHEAVES ON HYPERKÄHLER VARIETIES}

\subsection{Preliminary results}

Lemma 4.3. Let $(\Lambda, q)$ be a nondegenerate rank 2 lattice which represents 0 ; hence $\operatorname{disc}(\Lambda)=-d^{2}$, where $d$ is a strictly positive integer. Let $\alpha \in \Lambda$ be primitive isotropic, and complete it to a basis $\{\alpha, \beta\}$ such that $q(\beta) \geqslant 0$. If $\gamma \in \Lambda$ has strictly negative square (that is, $q(\gamma)<0)$, then

$$
q(\gamma) \leqslant-\frac{2 d}{1+q(\beta)} .
$$

Proof. There exist integers $x$ and $y$ such that $\gamma=x \alpha+y \beta$. Since $\operatorname{disc}(\Lambda)=-q(\alpha, \beta)^{2}$, we have $q(\alpha, \beta)=d$. Thus $q(\gamma)=y(2 d x+q(\beta) y)$. Since $q(\gamma)<0$ and since $x$ and $y$ are integers, we have

$$
0<|x|, \quad 0<|y| \leqslant|q(\gamma)|, \quad 0<|2 d x+q(\beta) y| \leqslant|q(\gamma)| .
$$

It follows that

$$
2 d|x|-q(\beta)|y| \leqslant|2 d x+q(\beta) y| \leqslant|q(\gamma)|
$$

because $d$ and $q(\beta)$ are nonnegative. Hence

$$
2 d \leqslant 2 d|x| \leqslant q(\beta)|y|+|2 d x+q(\beta) y| \leqslant q(\beta)|q(\gamma)|+|q(\gamma)|=(1+q(\beta))|q(\gamma)| .
$$

Since $q(\gamma)<0$, this inequality is equivalent to (4.2.1).

Proposition 4.4. Let $(A, \theta)$ be a principally polarized abelian surface. Let $\mathscr{F}$ be a $\theta$ slopesemistable vector bundle on $A$ such that $c_{1}(\mathscr{F})$ is a multiple of $\theta$ and $\Delta(\mathscr{F})=0$. Then we can write

$$
r(\mathscr{F})=r_{0}^{2} m, \quad c_{1}(\mathscr{F})=r_{0} b_{0} m \theta
$$

where $r_{0}, m, b_{0}$ are integers, the first two of which are positive, and $\operatorname{gcd}\left\{r_{0}, b_{0}\right\}=1$. If $\mathscr{F}$ is strictly $\theta$ slope-semistable, that is, not slope-stable, then there exists such a decomposition with $m>1$.

Proof. If $\mathscr{F}$ is slope-stable, then it is simple semi-homogeneous by Proposition A.2, and hence we may write (4.2.2) with $x=1$ by Proposition A.3.

Suppose that $\mathscr{F}$ is strictly $\theta$ slope-semistable. Then there exists a destabilizing exact sequence of torsion-free sheaves

$$
0 \longrightarrow \mathscr{G} \longrightarrow \mathscr{F} \longrightarrow \mathscr{H} \longrightarrow 0
$$

with $\mathscr{G}$ slope-stable. Notice that $\mathscr{G}$ is locally free because $\mathscr{H}$ is torsion-free.

Let us prove that $\mathscr{G}$ is simple semi-homogeneous. Since (4.2.3) is slope-destabilizing, $0<r(\mathscr{G})<r(\mathscr{F})$ and $\int_{A} \lambda_{\mathscr{G}, \mathscr{F}} \smile \theta=0$, where $\lambda_{\mathscr{G}, \mathscr{F}} \in H^{2}(A ; \mathbb{Z})$ is defined in (3.2.1). Since $\mathscr{F}$ is slope-semistable, $\mathscr{H}$ is slope-semistable. Thus $\Delta(\mathscr{G}) \geqslant 0$ and $\Delta(\mathscr{H}) \geqslant 0$ by Bogomolov's inequality. Now look at equation (3.3.2): since $\int_{A} \lambda_{\mathscr{G}, \mathscr{F}}^{2} \leqslant 0$ by the Hodge index theorem, we get that $\Delta(\mathscr{G})=\Delta(\mathscr{H})=0$ and $\int_{A} \lambda_{\mathscr{G}, \mathscr{F}}^{2}=0$. In particular, $\mathscr{G}$ is simple semi-homogeneous by Proposition A.2 and $\mathscr{H}$ is locally free (if it is not locally free, then $\mathscr{H}^{\vee}$ is a slope-semistable vector bundle with $\Delta\left(\mathscr{H}^{\vee}\right)<\Delta(\mathscr{H})=0$, but this contradicts Bogomolov's inequality). The equality $\int_{A} \lambda_{\mathscr{G}, \mathscr{F}}^{2}=0$ gives that $\lambda_{\mathscr{G}, \mathscr{F}}=0$ (by the Hodge index theorem). Thus $c_{1}(\mathscr{G})$ is a multiple of $\theta$, and so is $c_{1}(\mathscr{H})$.

Since the vector bundle $\mathscr{H}$ is slope-semistable, $c_{1}(\mathscr{H})$ is a multiple of $\theta$ and $\Delta(\mathscr{H})=0$, we can iterate this argument to get the following result. Let

$$
0=\mathscr{G}_{0} \subsetneq \mathscr{G}_{1} \subsetneq \cdots \subsetneq \mathscr{G}_{m}=\mathscr{F}
$$

be a Jordan-Hölder filtration (for slope-semistability) of $\mathscr{F}$. Then for $i \in\{1, \ldots, m\}$, the quotient $\mathscr{G}_{i} / \mathscr{G}_{i-1}$ is a simple semi-homogeneous vector bundle and $c_{1}\left(\mathscr{G}_{i} / \mathscr{G}_{i-1}\right)$ is a multiple of $\theta$. Let 


\section{K. G. O'GRADY}

$i \in\{1, \ldots, m\}$; by Proposition A.3, we may write

$$
r\left(\mathscr{G}_{i} / \mathscr{G}_{i-1}\right)=r_{i}^{2}, \quad c_{1}\left(\mathscr{G}_{i} / \mathscr{G}_{i-1}\right)=r_{i} b_{i} \theta
$$

where $r_{i}$ and $b_{i}$ are integers, $r_{i}>0$ and $\operatorname{gcd}\left\{r_{i}, b_{i}\right\}=1$. Let $i, j \in\{1, \ldots, m\}$; equating the slopes of $\mathscr{G}_{i} / \mathscr{G}_{i-1}$ and $\mathscr{G}_{j} / \mathscr{G}_{j-1}$, we get that

$$
\frac{b_{i}}{r_{i}}=\frac{b_{j}}{r_{j}}
$$

Since $\operatorname{gcd}\left\{r_{i}, b_{i}\right\}=\operatorname{gcd}\left\{r_{j}, b_{j}\right\}=1$, it follows that $r_{i}=r_{j}$ and $b_{i}=b_{j}$. Thus $r(\mathscr{F})=m r_{0}^{2}$ and $c_{1}(\mathscr{F})=m r_{0} b_{0} \theta$, where $r_{0}=r_{i}$ and $b_{0}=b_{i}$ for all $i \in\{1, \ldots, m\}$.

Corollary 4.5. Let $(A, \theta)$ be a principally polarized abelian surface. Let $\mathscr{F}$ be a $\theta$ slopesemistable vector bundle on $A$ such that $\Delta(\mathscr{F})=0$. If $r(\mathscr{F})=r_{0}^{2}$ and $c_{1}(\mathscr{F})=r_{0} b_{0} \theta$, where $r_{0}$ and $b_{0}$ are coprime integers, then $\mathscr{F}$ is $\theta$ slope-stable.

Proof. The proof is by contradiction. Suppose that $\mathscr{F}$ is not $\theta$ slope-stable. By Proposition 4.4, we may write $r(\mathscr{F})=s_{0}^{2} m$ and $c_{1}(\mathscr{F})=s_{0} c_{0} m \theta$, where $s_{0}, m, c_{0}$ are integers (with $s_{0}, m>0$ ), $s_{0}$ and $c_{0}$ are coprime and $m>1$. It follows that $s_{0} b_{0}=c_{0} r_{0}$. Since $\operatorname{gcd}\left\{r_{0}, b_{0}\right\}=1$ and $\operatorname{gcd}\left\{s_{0}, c_{0}\right\}=1$, we get that $r_{0}=s_{0}$ and hence $m=1$. This gives a contradiction.

4.3 Proof of item (i) of Proposition 4.1. First we prove that $h$ is $a_{0}$-suitable (see Definition 3.5). First suppose that $\rho(X)=2$, that is, $H_{\mathbb{Z}}^{1,1}(X)=\langle h, f\rangle$, where $f:=\pi^{*} c_{1}\left(\mathscr{O}_{\mathbb{P}^{2}}(1)\right)$. Apply Lemma 4.3 to $\Lambda:=H_{\mathbb{Z}}^{1,1}(X), \alpha=f$ and $\beta=h$ : by (4.1.1) we get that there are no $\xi \in H_{\mathbb{Z}}^{1,1}(X)$ such that $-a_{0} \leqslant q(\xi)<0$. Hence every ample divisor on $X$ is $a_{0}$-suitable.

Once we know that $h$ on $X$ is $a_{0}$-suitable if $\rho(X)=2$, it follows that the set of $[(X, h)] \in$ $\mathscr{N}_{e}^{i}(d)^{0}$ such that $h$ is not $a_{0}$-suitable belongs to the intersection of $\mathscr{N}_{e}^{i}(d)^{0}$ with a finite union of Noether-Lefschetz divisors in $\mathscr{K}_{e}^{i}$. In fact, suppose that $h$ is not $a_{0}$-suitable on $X$. Then there exists a $\gamma \in H_{\mathbb{Z}}^{1,1}(X)$ such that

$$
-a_{0} \leqslant q(\gamma)<0, \quad q(\gamma, h)>0, \quad q(\gamma, f)<0 .
$$

Let $B$ be the (finite) index of $\langle h, f\rangle \oplus\left(\langle h, f\rangle^{\perp} \cap H_{\mathbb{Z}}^{1,1}(X)\right)$ in $H_{\mathbb{Z}}^{1,1}(X)$. Then

$$
\gamma=\frac{\gamma_{1}}{B}+\frac{\gamma_{2}}{B}, \quad \gamma_{1} \in\langle h, f\rangle, \quad \gamma_{2} \in\langle h, f\rangle^{\perp} .
$$

By the last two inequalities in (4.3.1), we have $q\left(\gamma_{1}\right)<0$. Hence by the first inequality in (4.3.1), it follows that there exists a positive $M$ independent of $(X, h)$ such that $-M \leqslant q\left(\gamma_{2}\right)<0$. Hence the moduli point of $(X, h)$ belongs to the intersection of $\mathscr{N}_{e}^{i}(d)^{0}$ with a finite union of Noether-Lefschetz divisors in $\mathscr{K}_{e}^{i}$, as claimed.

We have proved that if $(X, h)$ represents a generic point of $\mathscr{N}_{e}^{i}(d)^{0}$, then $h$ is $a_{0}$-suitable, and hence $a(\mathscr{E})$-suitable because $a(\mathscr{E}) \leqslant a_{0}$. Let $A$ be a generic (smooth) fiber of $\pi$. By Proposition 3.6, the restriction of $\mathscr{E}$ to $A$ is slope-semistable with respect to the restriction of $h$.

We claim that the hypotheses of Corollary 4.5 are satisfied by $\mathscr{F}:=\mathscr{E} \mid A$. In fact, $\Delta(\mathscr{F})=0$ because $\mathscr{E}$ is modular; see Lemma 2.5. Moreover, the restriction of $h$ to $A$ is a multiple of a principal polarization $\theta$, by [Wie16, Theorem 1.1]. From the formula $\int_{A} h^{2}=\int_{X} h^{2} \smile f^{2}=$ $2 q(h, f)^{2}=2 d^{2}$, it follows that $\left.h\right|_{A}=d \theta$. Hence $r(\mathscr{F})=(m i)^{2}$ and $c_{1}(\mathscr{F})=m \cdot d \theta=(m i) d / i$. It follows that the hypotheses of Corollary 4.5 are satisfied and hence $\mathscr{F}$ is slope-stable.

4.4 Proof of item (ii) of Proposition 4.1. For $z \in \mathbb{P}^{2}$, we let $A_{z}:=\pi^{-1}(z), \mathscr{F}_{z}:=\mathscr{F}_{\mid} A_{z}$ and $\mathscr{G}_{z}:=\mathscr{G}_{\mid A_{z}}$. By item (i) of Proposition 4.1, there exists an open dense $U \subset \mathbb{P}^{2}$ such that for 


\section{MODULAR SHEAVES ON HYPERKÄHLER VARIETIES}

$z \in U$, the vector bundles $\mathscr{F}_{z}$ and $\mathscr{G}_{z}$ are both slope-stable. We claim that if $z \in U$, then $\mathscr{F}_{z}$ and $\mathscr{G}_{z}$ are simple semi-homogeneous vector bundles. In fact, they are simple because they are slope-stable, and they are semi-homogeneous by Lemma 2.5 and Proposition A.2. Let $z \in U$. By [Muk78, Theorem 7.11], the set

$$
V_{z}:=\left\{[\xi] \in A_{z}^{\vee} \mid \mathscr{F}_{z} \cong \mathscr{G}_{z} \otimes \xi\right\}
$$

is not empty, and hence it has cardinality $r(\mathscr{G})^{2}$ by Proposition 7.1 in op. cit. Clearly $V_{z}$ is invariant under the monodromy action of $\pi_{1}(U, z)$. Now notice that $V_{z} \subset A_{z}\left[(m i)^{2}\right]$ because $\mathscr{F}_{z}$ and $\mathscr{G}_{z}$ have rank $(m i)^{2}$ and isomorphic determinants. Hence by Corollary B.5, we have $V_{z}=A[m i]$. Thus $0 \in V_{z}$, and therefore $\mathscr{F}_{z} \cong \mathscr{G}_{z}$.

\section{Basic modular sheaves on Hilbert squares of $K 3$ surfaces}

5.1. Main results. Let $S$ be a smooth projective surface. Let $X_{n}(S) \rightarrow S^{n}$ be the blow-up of the big diagonal, that is, the $n$th isospectral Hilbert scheme of $S$; see [Hai01, Definition 3.2.4 and Proposition 3.4.2]. The complement of the big diagonal in $S^{n}$ is identified with a dense open subset $U_{n}(S)$ of $X_{n}(S)$, and the natural map $U_{n}(S) \rightarrow S^{[n]}$ extends to a regular map $p: X_{n}(S) \rightarrow S^{[n]}$ (this follows from [Hai01, Proposition 3.4.2]). Let $\tau: X_{n}(S) \rightarrow S^{n}$ be the blowup map. We let $q_{i}: X_{n}(S) \rightarrow S$ be the composition of $\tau$ and the $i$ th projection $S^{n} \rightarrow S$. Given a locally free sheaf $\mathscr{F}$ on $S$, let

$$
X_{n}(\mathscr{F}):=q_{1}^{*}(\mathscr{F}) \otimes \cdots \otimes q_{n}^{*}(\mathscr{F}) .
$$

The action of the symmetric group $\mathscr{S}_{n}$ on $S^{n}$ by permutation of the factors maps the big diagonal to itself and hence lifts to an action $\rho_{n}: \mathscr{S}_{n} \rightarrow \operatorname{Aut}\left(X_{n}(S)\right)$. The latter action lifts to a natural action $\rho_{n}^{+}$on $X_{n}(\mathscr{F})$. There is also a twisted action $\rho_{n}^{-}=\rho_{n}^{+} \cdot \chi$, where $\chi: \mathscr{S}_{n} \rightarrow\{ \pm 1\}$ is the sign character. Since $\rho_{n}$ maps to itself any fiber of $p: X_{n}(S) \rightarrow S^{[n]}$, the action $\rho_{n}^{ \pm}$descends to an action $\bar{\rho}_{n}^{ \pm}: \mathscr{S}_{n} \rightarrow \operatorname{Aut}\left(p_{*} X_{n}(\mathscr{F})\right)$.

Definition 5.1. Let $\mathscr{F}^{ \pm}[n] \subset p_{*} X_{n}(\mathscr{F})$ be the sheaf of $\mathscr{S}_{n}$-invariants for $\bar{\rho}_{n}^{ \pm}$.

Below is the first main result of the present section.

Proposition 5.2. Let $S$ be a projective $K 3$ surface, and let $\mathscr{F}$ be a locally free sheaf on $S$ such that $\chi(S, \operatorname{End}(\mathscr{F}))=2$. Then $\mathscr{F}[2]^{ \pm}$is a locally free modular sheaf of rank $r(\mathscr{F})^{2}$, with

$$
\begin{aligned}
\Delta\left(\mathscr{F}[2]^{ \pm}\right) & =\frac{r\left(\mathscr{F}[2]^{ \pm}\right)\left(r\left(\mathscr{F}[2]^{ \pm}\right)-1\right)}{12} c_{2}\left(S^{[2]}\right), \\
d\left(\mathscr{F}[2]^{ \pm}\right) & =5 \cdot\left(\begin{array}{c}
r\left(\mathscr{F}[2]^{ \pm}\right) \\
2
\end{array}\right), \\
a\left(\mathscr{F}[2]^{ \pm}\right) & =\frac{5}{8} r(\mathscr{F})^{6}\left(r(\mathscr{F})^{2}-1\right) .
\end{aligned}
$$

(Recall that $d(\mathscr{E})$ is defined by the equality in (1.2.2).)

The proof of Proposition 5.2 is given in Subsection 5.4.

Remark 5.3. If $S$ is a $K 3$ surface and $\mathscr{F}$ is a locally free sheaf on $S$ such that $\chi(S, \operatorname{End}(\mathscr{F})) \neq 2$, then $\mathscr{F}[2]^{ \pm}$is not modular.

The second main result of the section is the following. 


\section{K. G. O'GRADY}

Proposition 5.4. Let $S$ be a projective $K 3$ surface. Let $\mathscr{F}$ be a locally free sheaf on $S$ which is spherical, that is, such that $h^{p}\left(S, \operatorname{End}^{0}(\mathscr{F})\right)=0$ for all $p$, where $\operatorname{End}^{0}(\mathscr{F}) \subset \operatorname{End}(\mathscr{F})$ is the subsheaf of traceless endomorphisms. Then for all $p$, we have

$$
h^{p}\left(S^{[2]}, \operatorname{End}^{0}\left(\mathscr{F}[2]^{ \pm}\right)\right)=0 .
$$

The proof of Proposition 5.4 is in Subsection 5.5.

Below is a remarkable consequence of Proposition 5.4.

Corollary 5.5. Let $S$ be a projective $K 3$ surface, and let $\mathscr{F}$ be a locally free sheaf on $S$ which is spherical. Then the natural map between deformation spaces $\operatorname{Def}\left(S^{[2]}, \mathscr{F}[2]^{ \pm}\right) \longrightarrow$ $\operatorname{Def}\left(S^{[2]}, \operatorname{det} \mathscr{F}[2]^{ \pm}\right)$is smooth.

Proof. This follows from Proposition 5.4 and the main result of [IM19].

5.2. Another description of $\mathscr{F}[2]^{ \pm}$. A different definition of the sheaf $\mathscr{F}[2]^{-}$was given in [DHOV20]. Here we recall that construction, and we give the analogous construction of $\mathscr{F}[2]^{+}$.

The isospectral Hilbert scheme $X_{2}(S)$ is the the blow-up of the diagonal in $S \times S$; we will denote it by $\widetilde{S \times S}$. Let $E$ be the exceptional divisor of the blow-up map $\tau: \widetilde{S \times S} \rightarrow S^{2}$, and let $e \in H^{2}(\widetilde{S \times S})$ be its Poincaré dual. We let $\tau_{E}: E \rightarrow S$ be the restriction of $\tau$ to $E$. Let $\iota \in$ Aut $(\widetilde{S \times S})$ be the involution lifting the involution of $S^{2}$ exchanging the factors. Then $S^{[2]}$ is the quotient of $\widetilde{S \times S}$ by the group $\langle\iota\rangle$, and $p: \widetilde{S \times S} \rightarrow S^{[2]}$ is the quotient map. We recall that the map $q_{i}: \widetilde{S \times S} \rightarrow S$ for $i \in\{1,2\}$ is the composition of $\tau$ and the $i$ th projection $S \times S \rightarrow S$.

The natural map $f^{ \pm}: p^{*}\left(\mathscr{F}[2]^{ \pm}\right) \rightarrow q_{1}^{*} \mathscr{F} \otimes q_{2}^{*} \mathscr{F}$ is an isomorphism away from $E$; in particular, it is injective because $p^{*}\left(\mathscr{F}[2]^{ \pm}\right)$is torsion-free. In order to write out the cokernel, we notice that there are surjective morphisms

$$
q_{1}^{*} \mathscr{F} \otimes q_{2}^{*} \mathscr{F} \stackrel{\mathrm{ev}^{+}}{\longrightarrow} \tau_{E}^{*} \operatorname{Sym}^{2} \mathscr{F}, \quad q_{1}^{*} \mathscr{F} \otimes q_{2}^{*} \mathscr{F} \stackrel{\mathrm{ev}^{-}}{\longrightarrow} \tau_{E}^{*} \bigwedge^{2} \mathscr{F}
$$

obtained by evaluating along $E$ and then projecting onto the symmetric/antisymmetric part of $\left.\left(q_{1}^{*} \mathscr{F} \otimes q_{2}^{*} \mathscr{F}\right)\right|_{E}=\tau_{E}^{*}(\mathscr{F} \otimes \mathscr{F})$. Let $\iota: E \hookrightarrow \widetilde{S \times S}$ be the inclusion.

Proposition 5.6 ([DHOV20, Lemma 4.2]). The sheaves $\mathscr{F}[2]^{ \pm}$are locally free of rank $r(\mathscr{F})^{2}$, and the following are exact sequences:

$$
\begin{aligned}
& 0 \longrightarrow p^{*}\left(\mathscr{F}[2]^{+}\right) \stackrel{f^{+}}{\longrightarrow} q_{1}^{*} \mathscr{F} \otimes q_{2}^{*} \mathscr{F} \stackrel{\mathrm{ev}^{-}}{\longrightarrow} \iota_{*} \tau_{E}^{*}\left(\bigwedge^{2} \mathscr{F}\right) \longrightarrow 0 \\
& 0 \longrightarrow p^{*}\left(\mathscr{F}[2]^{-}\right) \stackrel{f^{-}}{\longrightarrow} q_{1}^{*} \mathscr{F} \otimes q_{2}^{*} \mathscr{F} \stackrel{\mathrm{ev}^{+}}{\longrightarrow} \iota_{*} \tau_{E}^{*} \operatorname{Sym}^{2} \mathscr{F} \longrightarrow 0
\end{aligned}
$$

Proof. Away from $E$, the sheaves $\iota_{*} \tau_{E}^{*}\left(\bigwedge^{2} \mathscr{F}\right)$ and $\iota_{*} \tau_{E}^{*}$ Sym ${ }^{2} \mathscr{F}$ are zero and the maps $f^{ \pm}$are isomorphisms. Hence (5.2.2) and (5.2.3) are exact away from $E$. In particular, $\mathscr{F}[2]^{ \pm}$is locally free of rank $r(\mathscr{F})^{2}$ away from $E$.

Let $x \in E$; then the subscheme $y:=p(x) \subset S$ is nonreduced, and hence it is supported at a single point $y_{\text {red }}$. Let $h \in \mathscr{O}_{\widetilde{S \times S}, x}$ be a local generator of the ideal of $E$. Let $\mathscr{F}\left(y_{\text {red }}\right)$ be the fiber of $\mathscr{F}$ at $y_{\text {red }}$. The \pm 1 eigenspaces for the action of $\rho_{2}^{+}$on $p_{*}\left(q_{1}^{*} \mathscr{F} \otimes q_{2}^{*} \mathscr{F}\right)_{y}$ are, respectively,

$$
\left(\operatorname{Sym}^{2} \mathscr{F}\left(y_{\text {red }}\right) \otimes \mathscr{O}_{S^{[2]}, y}\right) \oplus\left(\bigwedge^{2} \mathscr{F}\left(y_{\text {red }}\right) \otimes \mathscr{O}_{S^{[2]}, y} \cdot h\right)
$$




\section{MODUlAR SHEAVES ON HYPERKÄHLER VARIETIES}

and

$$
\left(\bigwedge^{2} \mathscr{F}\left(y_{\text {red }}\right) \otimes \mathscr{O}_{S^{[2]}, y}\right) \oplus\left(\operatorname{Sym}^{2} \mathscr{F}\left(y_{\text {red }}\right) \otimes \mathscr{O}_{S^{[2]}, y} \cdot h\right) .
$$

Thus $\mathscr{F}[2]_{x}^{+}$is free of rank $r(\mathscr{F})^{2}$, and we get that $(5.2 .2)$ is exact at $x$. Since the \pm eigenvalues of the action of $\rho_{2}^{-}$are the $\mp$ eigenvalues of the action of $\rho_{2}^{+}$, we also get that $\mathscr{F}[2]_{x}^{-}$is free of rank $r(\mathscr{F})^{2}$ and that $(5.2 .3)$ is exact at $x$.

5.3. Preliminaries on $K \mathbf{3}^{[n]}$. Let $\mu_{n}: H^{2}(S) \rightarrow H^{2}\left(S^{[n]}\right)$ be the composition of the natural symmetrization map $H^{2}(S) \rightarrow H^{2}\left(S^{(n)}\right)$ and the pull-back $H^{2}\left(S^{(n)}\right) \rightarrow H^{2}\left(S^{[n]}\right)$ defined by the Hilbert-Chow map $S^{[n]} \rightarrow S^{(n)}$. Let $\Delta^{[n]} \subset S^{[n]}$ be the prime divisor parametrizing nonreduced subschemes. The class $\mathrm{cl}\left(\Delta^{[n]}\right)$ is divisible by 2 in the integral cohomology of $S^{[n]}$; let $\delta_{n} \in$ $H_{\mathbb{Z}}^{1,1}\left(S^{[n]}\right)$ be the unique class such that $2 \delta_{n}=\operatorname{cl}\left(\Delta^{[n]}\right)$. We have an orthogonal decomposition for the BBF quadratic form

$$
H^{2}\left(S^{[n]} ; \mathbb{Z}\right)=\mu_{n}\left(H^{2}(S ; \mathbb{Z})\right) \oplus \mathbb{Z} \delta_{n}
$$

Let $q$ be the BBF form of $S^{[n]}$. Then for $\alpha \in H^{2}(S)$, we have

$$
q\left(\mu_{n}(\alpha)\right)=\int_{S} \alpha^{2}, \quad q\left(\mu_{n}(\alpha), \delta_{n}\right)=0, \quad q\left(\delta_{n}\right)=-2(n-1) .
$$

We will deal with $S^{[2]}$. In order to simplify notation, we will drop the subscripts of $\delta_{2}$ and $\mu_{2}$. We go through a few formulas that will be needed in the proof that $\mathscr{F}[2]^{ \pm}$is a modular sheaf. Let $\eta \in H^{4}(S ; \mathbb{Z})$ be the orientation class. We claim that

$$
\begin{gathered}
p^{*}\left(x \mu\left(c_{1}(\mathscr{F})\right)-y \delta\right)=x\left(q_{1}^{*} c_{1}(\mathscr{F})+q_{2}^{*} c_{1}(\mathscr{F})\right)-y e, \\
p^{*} c_{2}\left(S^{[2]}\right)=24\left(q_{1}^{*} \eta+q_{2}^{*} \eta\right)-3 e^{2} .
\end{gathered}
$$

In fact (5.3.2) follows directly from the definitions, and (5.3.3) is the last equation in [DV10, Proof of Lemma 4.5, p. 84]. Equation (5.3.3) gives

$$
\int_{S^{[2]}} c_{2}\left(S^{[2]}\right)^{2}=828, \quad \int_{S^{[2]}} c_{2}\left(S^{[2]}\right) \smile \alpha^{2}=30 q(\alpha), \quad \alpha \in H^{2}\left(S^{[2]}\right) .
$$

Lemma 5.7. Let $S$ be a $K 3$ surface. Let $\alpha \in H^{2}(S)$, and let $\alpha^{2}=2 m_{0} \eta$. Then

$$
2\left(q_{1}^{*} \eta \smile q_{2}^{*} \alpha+q_{1}^{*} \alpha \smile q_{2}^{*} \eta\right)+\left(q_{1}^{*} \alpha+q_{2}^{*} \alpha\right) \smile e^{2}=0 .
$$

Proof. Since the cohomology of $\widetilde{S \times S}$ has no torsion, it suffices to check that the cup product on the left-hand side of (5.3.5) with any class in $H^{2}(\widetilde{S \times S})$ vanishes. Thus we must take the cup product with $q_{i}^{*} \beta$, where $i \in\{1,2\}$ and $\beta \in H^{2}(S)$, and with $e$. The easy computations are left to the reader.

\subsection{Chern classes of $\mathscr{F}[2]^{ \pm}$}

Proposition 5.8. Let $S$ be a $K 3$ surface, and let $\mathscr{F}$ be a locally free sheaf of rank $r_{0}$ on $S$ such that $\chi(S, \operatorname{End}(\mathscr{F}))=2$. Let $h^{ \pm} \in H_{\mathbb{Q}}^{1,1}\left(S^{[2]}\right)$ be defined by

$$
h^{ \pm}:=\mu\left(c_{1}(\mathscr{F})\right)-\frac{r_{0} \mp 1}{2} \delta .
$$




\section{K. G. O'Grady}

Then

$$
\begin{aligned}
\operatorname{ch}_{0}\left(\mathscr{F}[2]^{ \pm}\right) & =r_{0}^{2} \\
\operatorname{ch}_{1}\left(\mathscr{F}[2]^{ \pm}\right) & =r_{0} h^{ \pm}, \\
\operatorname{ch}_{2}\left(\mathscr{F}[2]^{ \pm}\right) & =\frac{1}{2}\left(h^{ \pm}\right)^{2}-\frac{r_{0}^{2}-1}{24} c_{2}\left(S^{[2]}\right) .
\end{aligned}
$$

Proof. Of course (5.4.2) needs no proof. Let $\operatorname{ch}_{1}(\mathscr{F})^{2}=2 m_{0} \eta$, where $\eta \in H^{4}(S ; \mathbb{Z})$ is the orientation class. Since $\chi(S, \operatorname{End}(\mathscr{F}))=2$, the Hirzebruch-Riemann-Roch (HRR) theorem gives that

$$
2 r_{0} \operatorname{ch}_{2}(\mathscr{F})=\operatorname{ch}_{1}(\mathscr{F})^{2}-2\left(r_{0}^{2}-1\right) \eta=\left(2 m_{0}-2\left(r_{0}^{2}-1\right)\right) \eta .
$$

A straightforward computation shows that

$$
2 m_{0}=q\left(h^{ \pm}\right)+\frac{\left(r_{0} \mp 1\right)^{2}}{2} .
$$

Since the pull-back $p^{*}: H\left(S^{[2]} ; \mathbb{Z}\right) \rightarrow H^{2}(\widetilde{S \times S} ; \mathbb{Z})$ is injective, we work on $\widetilde{S \times S}$. By $(5.2 .2)$ and the Grothendieck-Riemann-Roch (GRR) theorem, we have

$$
\begin{aligned}
p^{*} & \operatorname{ch}\left(\mathscr{F}[2]^{+}\right) \\
= & q_{1}^{*} \operatorname{ch}(\mathscr{F}) \cdot q_{2}^{*} \operatorname{ch}(\mathscr{F}) \\
& -\iota_{*}\left(\tau_{E}^{*}\left(\left(\begin{array}{c}
r_{0} \\
2
\end{array}\right)+\left(r_{0}-1\right) \operatorname{ch}_{1}(\mathscr{F})+\left(r_{0}-2\right) \operatorname{ch}_{2}(\mathscr{F})+\frac{1}{2} \operatorname{ch}_{1}(\mathscr{F})^{2}\right)\right) \cdot\left(1-\frac{1}{2} e+\frac{1}{6} e^{2}-\frac{1}{24} e^{3}\right) \\
= & q_{1}^{*}\left(r_{0}+\operatorname{ch}_{1}(\mathscr{F})+\operatorname{ch}_{2}(\mathscr{F})\right) \cdot q_{2}^{*}\left(r_{0}+\operatorname{ch}_{1}(\mathscr{F})+\operatorname{ch}_{2}(\mathscr{F})\right) \\
& -\left(\left(\begin{array}{c}
r_{0} \\
2
\end{array}\right)+\frac{r_{0}-1}{2} \sum_{i=1}^{2} q_{i}^{*} \operatorname{ch}_{1}(\mathscr{F})+\frac{1}{4} \sum_{i=1}^{2}\left(q_{i}^{*}\left(\left(2 r_{0}-4\right) \operatorname{ch}_{2}(\mathscr{F})+\operatorname{ch}_{1}(\mathscr{F})^{2}\right)\right)\right) . \\
& \quad\left(e-\frac{1}{2} e^{2}+\frac{1}{6} e^{3}-\frac{1}{24} e^{4}\right) .
\end{aligned}
$$

(The cup product is denoted by · in order to save space.) Equation (5.4.3) for $\mathscr{F}[2]^{+}$follows at once. Using (5.4.5), we get that

$$
\begin{aligned}
p^{*} \operatorname{ch}_{2}\left(\mathscr{F}[2]^{+}\right)= & -\left(r_{0}^{2}-1\right)\left(q_{1}^{*} \eta+q_{2}^{*} \eta\right)+\frac{1}{2}\left(q_{1}^{*} \operatorname{ch}_{1}(\mathscr{F})^{2}+q_{2}^{*} \operatorname{ch}_{1}(\mathscr{F})^{2}\right) \\
& +q_{1}^{*} \operatorname{ch}_{1}(\mathscr{F}) \cdot q_{2}^{*} \operatorname{ch}_{1}(\mathscr{F})-\frac{r_{0}-1}{2} e \cdot\left(q_{1}^{*} \operatorname{ch}_{1}(\mathscr{F})+q_{2}^{*} \operatorname{ch}_{1}(\mathscr{F})\right)+\frac{1}{2}\left(\begin{array}{c}
r_{0} \\
2
\end{array}\right) e^{2}
\end{aligned}
$$

By (5.3.2) and (5.3.3), we get that (5.4.4) holds for $\mathscr{F}[2]^{+}$.

The computations for $\mathscr{F}[2]^{-}$are similar.

Now we prove Proposition 5.2. The sheaf $\mathscr{F}[2]^{ \pm}$is locally free of rank $r(\mathscr{F})^{2}$ by Proposition 5.6. Equation (5.1.1) holds by Proposition 5.8. It follows that $\mathscr{F}[2]^{ \pm}$is modular (see Remark 1.2). Equations (5.1.2) and (5.1.3) follow from (5.1.1) and the second equality in (5.3.4).

5.5 Cohomology groups via the MacKay correspondence. We keep the notation introduced in Subsection 5.2. In particular, $\tau: \overline{S \times S} \rightarrow S^{2}$ is the blow-up of the diagonal and $p: \widetilde{S \times S} \rightarrow S^{[2]}$ is the quotient map for the action of $\mathbb{Z} /(2)$ on $\overline{S \times S}$ which lifts the permutation action on $S^{2}$. Let $D_{\mathbb{Z} /(2)}^{b}\left(S^{2}\right)$ be the bounded derived category of the (abelian) category of $\mathbb{Z} /(2)$-equivariant coherent sheaves on $S^{2}$. By the MacKay correspondence proved by Haiman 


\section{MODULAR SHEAVES ON HYPERKÄHLER VARIETIES}

and Bridgeland-King-Reid, the functor

$$
p_{*}^{\mathbb{Z} /(2)} \circ \tau^{*}: D_{\mathbb{Z} /(2)}^{b}\left(S^{2}\right) \longrightarrow D^{b}\left(S^{[2]}\right)
$$

is an equivalence; see [Kru18, Proposition 2.8]. (Here $p_{*}^{\mathbb{Z} /(2)}$ is the derived functor of the functor $\operatorname{Coh}(\widetilde{S \times S}) \rightarrow \operatorname{Coh}\left(S^{[2]}\right)$ mapping $\mathscr{F}$ to the $\mathbb{Z} /(2)$-invariant subsheaf of $p_{*}(\mathscr{F})$.) We have

$$
p_{*}^{\mathbb{Z} /(2)} \circ \tau^{*}(\mathscr{F} \rrbracket 2)=\mathscr{F}^{+}, \quad p_{*}^{\mathbb{Z} /(2)} \circ \tau^{*}(\mathscr{F} \rrbracket 2 \otimes \mathfrak{a})=\mathscr{F}^{-},
$$

where $\mathfrak{a}$ is the sign representation of $\mathbb{Z} /(2)$. It follows by the Künneth formula that

$$
\operatorname{Ext}^{*}\left(\mathscr{F}^{ \pm}, \mathscr{F}^{ \pm}\right) \cong \operatorname{Ext}^{*}\left(\mathscr{F}{ }^{\bigotimes}, \mathscr{F}^{凶 2}\right)^{\mathbb{Z} /(2)} \cong \operatorname{Sym}^{2}\left(\operatorname{Ext}^{*}(\mathscr{F}, \mathscr{F})\right)
$$

We now prove Proposition 5.4. Since $H^{p}(S, \operatorname{End}(\mathscr{F}))=H^{p}\left(S, \operatorname{End}^{0}(\mathscr{F})\right) \oplus H^{p}\left(S, \mathscr{O}_{S}\right)$ and $S$ is a $K 3$ surface, the cohomology space $\operatorname{Ext}^{p}(\mathscr{F}, \mathscr{F})$ is 1-dimensional if $p \in\{0,2\}$ and vanishes otherwise. Hence

$$
\operatorname{Sym}^{2}\left(\operatorname{Ext}^{*}(\mathscr{F}, \mathscr{F})\right)=\operatorname{Sym}^{2}(\mathbb{C}[0] \oplus \mathbb{C}[-2])=\mathbb{C}[0] \oplus \mathbb{C}[-2] \oplus \mathbb{C}[-4] .
$$

By (5.5.3), we get that $H^{p}\left(S^{[2]}, \operatorname{End}(\mathscr{F})\right)$ is 1-dimensional if $p \in\{0,2,4\}$ and vanishes otherwise. Proposition 5.4 follows because $h^{p}\left(S^{[2]}, \mathscr{O}_{S^{[2]}}\right)=1$ for $p \in\{0,2,4\}$ and $H^{p}\left(S^{[2]}, \operatorname{End}\left(\mathscr{F}^{ \pm}\right)\right)=$ $H^{p}\left(S^{[2]}, \operatorname{End}^{0}\left(\mathscr{F}^{ \pm}\right)\right) \oplus H^{p}\left(S^{[2]}, \mathscr{O}_{[2]}\right)$.

\section{Basic modular sheaves on the Hilbert square of an elliptic $K 3$ surface}

6.1. Contents of the section. We will study the vector bundle $\mathscr{F}[2]^{+}$for a spherical vector bundle $\mathscr{F}$ on an elliptic $K 3$ surface $S$ with Picard number 2 (analogous results hold for $\mathscr{F}[2]^{-}$). Given positive integers $e, r_{0}, i$ satisfying the hypotheses of Theorem 1.4, one can choose suitable $S$ and $\mathscr{F}$ such that the equations in (1.3.4) hold for $\mathscr{E}:=\mathscr{F}[2]^{+}$—see Subsection 6.3. Next, notice that there is a Lagrangian fibration $\pi: S^{[2]} \rightarrow \mathbb{P}^{2}$ associated with the elliptic fibration $S \rightarrow \mathbb{P}^{1}$. In Subsection 6.4, we analyze the restriction of $\mathscr{F}[2]^{ \pm}$to (scheme-theoretic) fibers of $\pi$. The key result is Proposition 6.7, which states that the restriction to every Lagrangian fiber is simple. Along the way, we make another key observation: the restriction to a generic Lagrangian fiber is slope-stable; see Proposition 6.11.

6.2. Elliptic $K 3$ surfaces and stable rigid vector bundles. We recall the notions of Mukai vector and Mukai pairing for a $K 3$ surface $S$. If $\mathscr{F}$ is a sheaf over $S$, the Mukai vector of $\mathscr{F}$ is $v(\mathscr{F}):=\operatorname{ch}(\mathscr{F}) \operatorname{Td}(S)^{1 / 2}$. Moreover, the bilinear symmetric Mukai pairing $\langle\cdot, \cdot\rangle$ on $H(S)$ has the following property: if $\mathscr{F}$ and $\mathscr{E}$ are sheaves on $S$, then

$$
\langle v(\mathscr{F}), v(\mathscr{E})\rangle=-\chi(\mathscr{F}, \mathscr{E}):=-\sum_{i=0}^{2}(-1)^{i} \operatorname{dim} \operatorname{Ext}^{i}(\mathscr{F}, \mathscr{E}) .
$$

Let $S$ be a $K 3$ surface with an elliptic fibration $S \rightarrow \mathbb{P}^{1}$; we let $C$ be a fiber of the elliptic fibration. The claim below follows from the surjectivity of the period map for $K 3$ surfaces.

Claim 6.1. Let $m_{0}$ and $d_{0}$ be positive natural numbers. There exist $K 3$ surfaces $S$ with an elliptic fibration $S \rightarrow \mathbb{P}^{1}$ such that

$$
H_{\mathbb{Z}}^{1,1}(S)=\mathbb{Z}[D] \oplus \mathbb{Z}[C], \quad D \cdot D=2 m_{0}, \quad D \cdot C=d_{0} .
$$

The result below provides us with stable vector bundles $\mathscr{F}$ on elliptic $K 3$ surfaces such that $\mathscr{F}[2]^{ \pm}$has good properties; see Proposition 6.7 . 


\section{K. G. O'GRADY}

Proposition 6.2. Let $m_{0}, r_{0}, s_{0}$ be positive integers such that $m_{0}+1=r_{0} s_{0}$. Suppose that $d_{0}$ is an integer coprime to $r_{0}$ and that

$$
d_{0}>\frac{\left(2 m_{0}+1\right) r_{0}^{2}\left(r_{0}^{2}-1\right)}{4} .
$$

Let $S$ be an elliptic $K 3$ surface as in Claim 6.1. Then there exists a vector bundle $\mathscr{F}$ on $S$ such that the following hold:

(i) $v(\mathscr{F})=\left(r_{0}, D, s_{0}\right)$.

(ii) $\chi(S, \operatorname{End}(\mathscr{F}))=2$.

(iii) The vector bundle $\mathscr{F}$ is $L$ slope-stable for any polarization $L$ of $S$.

(iv) The restriction of $\mathscr{F}$ to every fiber of the elliptic fibration $S \rightarrow \mathbb{P}^{1}$ is slope-stable.

(Notice that every fiber is irreducible by our assumptions on $\operatorname{NS}(S)$, hence the slope-stability of a sheaf on a fiber is well defined, that is, independent of the choice of a polarization.)

Proof. (i) The Mukai vector $v=\left(r_{0}, D, s_{0}\right) \in H(S)$ has square -2 . Let $L_{0}$ be a polarization of $S$. By [Kul90, Theorem 2.1], there exists an $L_{0}$ slope-semistable vector bundle $\mathscr{F}$ on $S$ with $v(\mathscr{F})=\left(r_{0}, D, s_{0}\right)$.

(ii) We have that $\chi(S, \operatorname{End}(\mathscr{F}))=2$ because $v(\mathscr{F})^{2}=-2$.

(iii) We claim that $\mathscr{F}$ is actually $L_{0}$ slope-stable and that it is $L$ slope-stable for any polarization $L$ of $S$. This follows from the well-known results on the stability chamber decomposition of $\operatorname{Amp}(S)$, which have been extended to arbitrary HK varieties in Section 3. In fact, $v(\mathscr{F})^{2}=-2$ and (1.2.1) give that $\Delta(\mathscr{F})=2\left(r_{0}^{2}-1\right)$. It follows that $a(\mathscr{F})=\frac{1}{2} r_{0}^{2}\left(r_{0}^{2}-1\right)$, and hence by Lemma 4.3 and (6.2.3), there is no $a(\mathscr{F})$-wall. Thus there is a single $a(\mathscr{F})$-chamber.

(iv) By Proposition 3.6, the restriction of $\mathscr{F}$ to a generic fiber $C$ of the elliptic fibration is slope-semistable (because there is a single $a(\mathscr{F})$-chamber). Since $d_{0}$, which is the degree of $\mathscr{F}_{\mid C}$, is coprime to $r_{0}$, which is the rank of $\mathscr{F}_{\mid C}$, it follows that the restriction of $\mathscr{F}$ to a generic fiber $C$ is slope-stable. Suppose that there exists a fiber $C_{0}$ such that $\mathscr{F} \mid C_{0}$ is not slope-stable. Then $\mathscr{F}_{\mid C_{0}}$ is slope-unstable because $d_{0}$ is coprime to $r_{0}$. Let $\mathscr{F}_{\mid C_{0}} \rightarrow \mathscr{B}$ be a desemistabilizing quotient, that is, $0<r(\mathscr{B})<r$ and $\mu(\mathscr{B})-\mu\left(\mathscr{F} \mid C_{0}\right)<0$. Let $\mathscr{E}$ be the elementary modification of $\mathscr{F}$ associated with the quotient $\mathscr{B}$, that is, the (torsion-free) sheaf on $S$ fitting into the exact sequence

$$
0 \longrightarrow \mathscr{E} \longrightarrow \mathscr{F} \longrightarrow i_{0, *} \mathscr{B} \longrightarrow 0
$$

where $i_{0}: C_{0} \hookrightarrow S$ is the inclusion map. Then

$$
v(\mathscr{E})^{2}=v(\mathscr{F})^{2}+2 r_{0} \cdot r(\mathscr{B}) \cdot\left(\mu(\mathscr{B})-\mu\left(\mathscr{F} \mid C_{0}\right)\right)<v(\mathscr{F})^{2}=-2 .
$$

By (6.2.1), it follows that $\chi(\mathscr{E}, \mathscr{E})>2$. On the other hand, since $\mathscr{E}$ is isomorphic to $\mathscr{F}$ outside $C_{0}$, the restriction of $\mathscr{E}$ to a generic elliptic fiber is slope-stable, and this implies that $\operatorname{Hom}(\mathscr{E}, \mathscr{E})=$ $\mathbb{C} \operatorname{Id}_{\mathscr{E}}$. By Serre duality, it follows that $\operatorname{dim} \operatorname{Ext}^{2}(\mathscr{E}, \mathscr{E})=1$. The last two facts contradict the inequality $\chi(\mathscr{E}, \mathscr{E})>2$.

6.3. Dictionary. We show that, given positive integers $i, e, r_{0}$ satisfying the hypotheses of Theorem 1.4, we get a vector bundle $\mathscr{E}$ on the Hilbert square of a suitable $K 3$ surface such that the equations in (1.3.4) hold. First, we need the result below. The elementary proof is left to the reader. 


\section{MODUlAR SHEAVES ON HYPERKÄHLER VARIETIES}

Lemma 6.3. Let $i \in\{1,2\}$. Let $e$ and $r_{0}$ be positive natural numbers such that $r_{0} \equiv i(\bmod 2)$ and (1.3.3) holds. Let

$$
m_{0}:= \begin{cases}\frac{e}{2}+\frac{\left(r_{0}-1\right)^{2}}{4} & \text { if } r_{0} \text { is odd } \\ \frac{e}{8}+\frac{\left(r_{0}-1\right)^{2}}{4} & \text { if } r_{0} \text { is even }\end{cases}
$$

( $m_{0}$ is an integer by (1.3.3).) There exists an integer $s_{0}$ such that $m_{0}+1=r_{0} s_{0}$.

Let $i, e, r_{0}, m_{0}$ be as in Lemma 6.3. Suppose that $d_{0}$ is an integer coprime to $r_{0}$ such that (6.2.3) holds. Let $S$ be an elliptic $K 3$ surface as in Claim 6.1, and let $\mathscr{F}$ be a vector bundle on $S$ as in Proposition 6.2 (it exists by Lemma 6.3). Let $\mathscr{E}:=\mathscr{F}[2]^{+}$, and let $h^{+}$be as in (5.4.1). Lastly, let

$$
h:=i h^{+} .
$$

Proposition 6.4. Keeping the notation and hypotheses as above, the following hold:

(i) The cohomology class $h$ is primitive, $q(h)=e$, and $q\left(h, H^{2}(X ; \mathbb{Z})\right)=(i)$,

(ii) The values of $r(\mathscr{E}), c_{1}(\mathscr{E})$ and $\Delta(\mathscr{E})$ are given by (1.3.4).

Proof. Item (i) holds because $\operatorname{cl}(D)$ is primitive and by equation (5.4.6). Item (ii) follows from equations (5.4.2)-(5.4.4) and straightforward computations.

Remark 6.5. In Proposition 6.4 , we have set $\mathscr{E}:=\mathscr{F}[2]^{+}$. One gets an analogous result letting $\mathscr{E}=\mathscr{F}[2]^{-}$(one needs to replace $\frac{1}{4}\left(r_{0}-1\right)^{2}$ with $\frac{1}{4}\left(r_{0}+1\right)^{2}$ in $(6.3 .1)$ ).

\subsection{Restriction of $\mathscr{F}[2]^{ \pm}$to Lagrangian fibers}

Definition 6.6. If $S \rightarrow \mathbb{P}^{1}$ is an elliptically fibered $K 3$ surface, the associated Lagrangian fibration is the composition

$$
S^{[2]} \longrightarrow S^{(2)} \longrightarrow\left(\mathbb{P}^{1}\right)^{(2)} \cong \mathbb{P}^{2}
$$

In the present subsection, we prove the following result.

Proposition 6.7. Let $S$ be a $K 3$ surface with an elliptic fibration $S \rightarrow \mathbb{P}^{1}$ as in Claim 6.1, and let $\pi: S^{[2]} \rightarrow \mathbb{P}^{2}$ be the associated Lagrangian fibration. Let $\mathscr{F}$ be a vector bundle on $S$ as in Proposition 6.2. Then the restriction of $\mathscr{F}[2]^{ \pm}$to every (scheme-theoretic) fiber of $\pi$ is simple.

The proof is given at the end of the subsection.

Let $S$ and $\pi: S^{[2]} \rightarrow \mathbb{P}^{2}$ be as in Proposition 6.7. We describe the fibers of $\pi$.

For $x \in \mathbb{P}^{1}$, we let $C_{x}$ be the fiber over $x$ of the elliptic fibration $S \rightarrow \mathbb{P}^{1}$. The set-theoretic fibers of $\pi$ are as follows:

$$
\pi^{-1}\left(x_{1}+x_{2}\right)= \begin{cases}C_{x_{1}} \times C_{x_{2}} & \text { if } x_{1} \neq x_{2}, \\ C_{x}^{(2)} \cup \mathbb{P}\left(\Theta_{S \mid C_{x}}\right) & \text { if } x_{1}=x_{2}=x .\end{cases}
$$

As is easily checked, the fiber is reduced if $x_{1} \neq x_{2}$. On the other hand, the fiber over $2 x$ is not reduced. In order to prove this, we introduce some notation. Let $V^{[2]} \subset S^{[2]}$ be the prime divisor parametrizing vertical subschemes $Z$ (that is, such that the scheme-theoretic image $f(Z)$ is a reduced point), let $\Delta^{[2]} \subset S^{[2]}$ be the prime divisor parametrizing nonreduced subschemes, and let $D^{(2)} \subset\left(\mathbb{P}^{1}\right)^{(2)}$ be the prime divisor parametrizing multiple 0-cycles $2 x$. 


\section{K. G. O'Grady}

Proposition 6.8. Let $S$ be a $K 3$ surface with an elliptic fibration $S \rightarrow \mathbb{P}^{1}$ as in Claim 6.1. With notation as above, we have the equality of (Cartier) divisors $\pi^{*}\left(D^{(2)}\right)=2 V^{[2]}+\Delta^{[2]}$.

Proof. By the set-theoretic equality $\pi^{-1}\left(D^{(2)}\right)=V^{[2]} \cup \Delta^{[2]}$, there exist positive integers $a$ and $b$ such that $\pi^{*}\left(D^{(2)}\right)=a V^{[2]}+b \Delta^{[2]}$. We have a commutative square

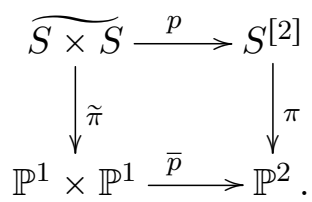

Let $\tilde{V}^{[2]}:=p^{-1}\left(V^{[2]}\right)$, and let $\operatorname{Diag}_{\mathbb{P}^{1}}$ be the diagonal of $\mathbb{P}^{1} \times \mathbb{P}^{1}$. The proposition follows from the equalities

$$
a \tilde{V}^{[2]}+2 b E=p^{*}\left(\pi^{*}\left(D^{(2)}\right)\right)=\tilde{\pi}^{*}\left(\bar{p}^{*}\left(D^{(2)}\right)\right)=\tilde{\pi}^{*}\left(2 \operatorname{Diag}_{\mathbb{P}^{1}}\right)=2 \tilde{V}^{[2]}+2 E .
$$

Corollary 6.9. Keep the hypotheses and notation as in Proposition 6.8. Let $\mathscr{L}$ be the line bundle on $S^{[2]}$ such that $c_{1}(\mathscr{L})=\delta$. Let $x \in \mathbb{P}^{1}$, and let $Z_{x}=\pi^{-1}(2 x)$ be the schematic fiber of $\pi$ over $2 x$. Then

$$
\mathscr{I}_{Z_{x}^{\text {red }} / Z_{x} \mid C_{x}^{(2)}} \cong \mathscr{O}_{C_{x}^{(2)}}\left(-\Xi_{x}\right) \otimes\left(\mathscr{L}_{\mid C_{x}^{(2)}}\right),
$$

where $\Xi_{x}:=\left\{2 p \mid p \in C_{x}\right\} \subset C_{x}^{(2)}$. In particular, the restriction homomorphism $\mathscr{O}_{Z_{x}} \rightarrow \mathscr{O}_{Z_{x}^{\text {red }}}$ fits into the exact sequence

$$
0 \longrightarrow \mathscr{O}_{C_{x}^{(2)}}\left(-\Xi_{x}\right) \otimes\left(\mathscr{L}_{\mid C_{x}^{(2)}}\right) \longrightarrow \mathscr{O}_{Z_{x}} \longrightarrow \mathscr{O}_{Z_{x}^{\text {red }}} \longrightarrow 0
$$

Proof. It suffices to prove that (6.4.4) holds because the kernel of the surjection $\mathscr{O}_{Z_{x}} \rightarrow \mathscr{O}_{Z_{x}^{\text {red }}}$ is equal to the left-hand side in (6.4.4). By Proposition 6.8, we have

$$
\mathscr{I}_{Z_{x}^{\text {red }} / Z_{x} \mid C_{x}^{(2)}} \cong \mathscr{O}_{S^{[2]}}\left(-V^{[2]}-\Delta^{[2]}\right)_{\mid C_{x}^{(2)}} .
$$

We have

$$
\mathscr{O}_{S^{[2]}}\left(-\Delta^{[2]}\right)_{\mid C_{x}^{(2)}} \cong \mathscr{O}_{C_{x}^{(2)}}\left(-\Xi_{x}\right)
$$

On the other hand, Proposition 6.8 gives that

$$
\mathscr{O}_{S^{[2]}}\left(2 V^{[2]}\right) \cong \mathscr{O}_{S^{[2]}}\left(\pi^{*} D^{(2)}-\Delta^{[2]}\right) \cong \pi^{*}\left(\mathscr{O}_{\mathbb{P}^{2}}(2)\right) \otimes \mathscr{L}^{-2} .
$$

Since $H^{1}\left(S^{[2]} ; \mathbb{Z}\right)=0$, it follows that $\mathscr{O}_{S^{[2]}}\left(V^{[2]}\right) \cong \pi^{*}\left(\mathscr{O}_{\mathbb{P}^{2}}(1)\right) \otimes \mathscr{L}^{-1}$. Since the restriction of $\mu\left(c_{1}\left(\mathscr{O}_{\mathbb{P}^{2}}(1)\right)\right)$ to $C_{x}^{(2)}$ is trivial, we get that

$$
\mathscr{O}_{S^{[2]}}\left(-V^{[2]}\right)_{\mid C_{x}^{(2)}} \cong \mathscr{L}_{\mid C_{x}^{(2)}}
$$

Hence (6.4.4) follows from (6.4.6), (6.4.7) and (6.4.8).

For $x \in \mathbb{P}^{1}$, let $\mathscr{F}_{x}:=\mathscr{F}_{\mid} C_{x}$. If $x_{1} \neq x_{2} \in \mathbb{P}^{1}$, then the restriction of $\mathscr{F}$ to $\pi^{-1}\left(x_{1}+x_{2}\right)$ is equal to $\mathscr{F}_{x_{1}} \bigotimes \mathscr{F}_{x_{2}}$. Thus we need the following result.

Proposition 6.10. Let $C_{i}$, for $i \in\{1,2\}$, be polarized irreducible curves, and let $D$ be an ample divisor on $Y:=C_{1} \times C_{2}$ such that

$$
c_{1}\left(\mathscr{O}_{Y}(D)\right)=m_{1} \rho_{1}^{*} \mathscr{O}_{Y_{1}}\left(p_{1}\right)+m_{2} \rho_{2}^{*} c_{1}\left(\mathscr{O}_{Y_{2}}\left(p_{2}\right)\right),
$$

where $\rho_{i}: Y \rightarrow C_{i}$ is the projection and $p_{i} \in C_{i}$. Let $\mathscr{V}_{i}$ be a slope-stable vector bundle on $C_{i}$ for $i \in\{1,2\}$. Then $\mathscr{V}_{1} \bowtie \mathscr{V}_{2}$ is $D$ slope-stable. 


\section{MODUlAR SHEAVES ON HYPERKÄHLER VARIETIES}

Proof. The proof is by contradiction. Suppose the contrary. Then there exists an injection $\alpha: \mathscr{E} \rightarrow$ $\mathscr{V}_{1} \bowtie \mathscr{V}_{2}$ with torsion-free cokernel such that $0<r(\mathscr{E})<r\left(\mathscr{V}_{1} \bowtie \mathscr{V}_{2}\right)$ and

$$
\mu_{D}(\mathscr{E}) \geqslant \mu_{D}\left(\mathscr{V}_{1} \bowtie \mathscr{V}_{2}\right)
$$

Let $p_{i} \in C_{i}$ be generic; the restrictions of $\alpha$ to $\left\{p_{1}\right\} \times C_{2}$ and $C_{1} \times\left\{p_{2}\right\}$ are injective maps of vector bundles (that is, injective on fibers). We have

$$
\mu_{D}(\mathscr{E})=m_{2} \mu\left(\mathscr{E}_{\mid C_{1} \times\left\{p_{2}\right\}}\right)+m_{1} \mu\left(\mathscr{E}_{\mid\left\{p_{1}\right\} \times C_{2}}\right) .
$$

On the other hand,

$$
\mu_{D}\left(\mathscr{V}_{1} \bowtie \mathscr{V}_{2}\right)=m_{2} \mu\left(\mathscr{V}_{1}\right)+m_{1} \mu\left(\mathscr{V}_{2}\right)
$$

Since the restrictions of $\mathscr{V}_{1} \otimes \mathscr{V}_{2}$ to $C_{1} \times\left\{p_{2}\right\}$ and $\left\{p_{1}\right\} \times C_{2}$ are isomorphic to the polystable vector bundles $\mathscr{V}_{1} \otimes_{\mathbb{C}} \mathbb{C}^{r\left(\mathscr{V}_{2}\right)}$ and $\mathscr{V}_{1} \otimes_{\mathbb{C}} \mathbb{C}^{r\left(\mathscr{V}_{1}\right)}$, respectively, it follows from (6.4.10), (6.4.11) and (6.4.12) that $\mu\left(\mathscr{E}_{\mid C_{1} \times\left\{p_{2}\right\}}\right)=\mu\left(\mathscr{V}_{1}\right)$ and $\mu\left(\mathscr{E}_{\mid\left\{p_{1}\right\} \times C_{2}}\right)=\mu\left(\mathscr{V}_{2}\right)$. In turn, these equalities give that there exist vector subspaces $0 \neq A \subset \mathbb{C}^{r(\mathscr{V} / 2)}$ and $0 \neq B \subset \mathbb{C}^{r(\mathscr{V} / 1)}$ such that $\mathscr{E}_{\mid} C_{1} \times\left\{p_{2}\right\}=\mathscr{V}_{1} \otimes_{\mathbb{C}} A$ and $\mathscr{E}_{\mid\left\{p_{1}\right\} \times C_{2}}=B \otimes_{\mathbb{C}} \mathscr{V}_{2}$. It follows that $\operatorname{Im} \alpha=\mathscr{V}_{1} \bigotimes \mathscr{V}_{2}$. This gives a contradiction.

Proposition 6.11. Let $S$ be a $K 3$ surface with an elliptic fibration $S \rightarrow \mathbb{P}^{1}$ as in Claim 6.1, and let $\mathscr{F}$ be a vector bundle on $S$ as in Proposition 6.2. If $x_{1} \neq x_{2}$, the restriction of $\mathscr{F}[2]^{ \pm}$ to $\pi^{-1}\left(x_{1}+x_{2}\right)=C_{x_{1}} \times C_{x_{2}}$ is slope-stable for any product polarization, and it is a simple semi-homogeneous vector bundle.

Proof. We have

$$
\mathscr{F}[2]_{\mid \pi^{*}\left(x_{1}+x_{2}\right)}^{ \pm} \cong \mathscr{F}_{x_{1}} \text { 囚 } \mathscr{F}_{x_{2}} .
$$

By Proposition 6.2 , both $\mathscr{F}_{x_{1}}$ and $\mathscr{F}_{x_{2}}$ are slope-stable. Hence the statement about slope-stability follows from Proposition 6.10. Of course, this implies that the restriction of $\mathscr{F}[2]^{ \pm}$to $\pi^{-1}\left(x_{1}+\right.$ $\left.x_{2}\right)=C_{x_{1}} \times C_{x_{2}}$ is simple. Moreover, it is semi-homogeneous because every stable vector bundle on an elliptic curve is semi-homogeneous. (One could argue that the discriminant vanishes by Lemma 2.5 and conclude by Proposition A.2.)

For $x \in \mathbb{P}^{1}$, let $\Delta_{x}^{[2]}:=\mathbb{P}\left(\Theta_{S \mid C_{x}}\right) \subset \Delta^{[2]}$, and let $\mathscr{O}_{\Delta_{x}^{[2]}}(1)$ be the dual of the tautological line subbundle $\Delta_{x}^{[2]}$. Let $\tau_{x}: \Delta_{x}^{[2]} \rightarrow C_{x}$ be the restriction of the Hilbert-Chow map.

Lemma 6.12. Let $S$ be a $K 3$ surface with an elliptic fibration $S \rightarrow \mathbb{P}^{1}$, and let $\mathscr{F}$ be a vector bundle on $S$ as in Proposition 6.2. Let $x$ be a regular value of the elliptic fibration. Then

$$
\begin{aligned}
& \mathscr{F}[2]_{\mid \Delta_{x}^{[2]}}^{+} \cong\left(\mathscr{O}_{\Delta_{x}^{[2]}}(1) \otimes \tau_{x}^{*} \bigwedge^{2} \mathscr{F}_{x}\right) \oplus \tau_{x}^{*}\left(\operatorname{Sym}^{2} \mathscr{F}_{x}\right), \\
& \mathscr{F}[2]_{\mid \Delta_{x}^{[2]}}^{-} \cong\left(\mathscr{O}_{\Delta_{x}^{[2]}}(1) \otimes \tau_{x}^{*} \operatorname{Sym}^{2} \mathscr{F}_{x}\right) \oplus \tau_{x}^{*}\left(\bigwedge^{2} \mathscr{F}_{x}\right),
\end{aligned}
$$

and the space of traceless endomorphisms of $\mathscr{F}[2]_{\mid \Delta_{x}^{[2]}}^{ \pm}$has dimension 1.

Proof. The proofs for $\mathscr{F}[2]^{ \pm}$are similar. We provide the proof for $\mathscr{F}[2]^{+}$. Restricting the defining exact sequence $(5.2 .2)$ to $E$, we get the exact sequence

$$
0 \longrightarrow \tau_{E}^{*}\left(\bigwedge^{2} \mathscr{F}\right) \otimes \mathscr{O}_{E}(-E) \longrightarrow \mathscr{F}[2]_{\mid E}^{+} \longrightarrow \tau_{E}^{*}\left(\operatorname{Sym}^{2} \mathscr{F}\right) \longrightarrow 0
$$




\section{K. G. O'GRADY}

Restricting the exact sequence in (6.4.16) to $\Delta_{x}^{[2]}$, we get the exact sequence

$$
0 \longrightarrow \tau_{x}^{*}\left(\bigwedge^{2} \mathscr{F}_{x}\right) \otimes \mathscr{O}_{\Delta_{x}^{[2]}}(1) \longrightarrow \mathscr{F}[2]_{\mid \Delta_{x}^{[2]}}^{+} \stackrel{\beta}{\longrightarrow} \tau_{x}^{*}\left(\operatorname{Sym}^{2} \mathscr{F}_{x}\right) \longrightarrow 0 .
$$

We claim that this exact sequence splits. The extension class belongs to

$$
H^{1}\left(\Delta_{x}^{[2]}, \tau_{x}^{*}\left(\operatorname{Sym}^{2} \mathscr{F}_{x}^{\vee} \otimes \bigwedge^{2} \mathscr{F}_{x}\right) \otimes \mathscr{O}_{\Delta_{x}^{[2]}}(1)\right) .
$$

We compute this cohomology group via the Leray spectral sequence of $\tau_{x}$. Since $\left(\operatorname{Sym}^{2} \mathscr{F}_{x}^{\vee}\right.$ $\left.\otimes \bigwedge^{2} \mathscr{F}_{x}\right) \otimes R^{1} \tau_{x, *} \mathscr{O}_{\Delta_{x}^{[2]}}(1)=0$, it suffices to show that

$$
\begin{aligned}
& H^{1}\left(C_{x},\left(\operatorname{Sym}^{2} \mathscr{F}_{x}^{\vee} \otimes \bigwedge^{2} \mathscr{F}_{x}\right) \otimes \tau_{x, *} \mathscr{O}_{\Delta_{x}^{[2]}}(1)\right) \\
& \quad=H^{1}\left(C_{x},\left(\operatorname{Sym}^{2} \mathscr{F}_{x}^{\vee} \otimes \bigwedge^{2} \mathscr{F}_{x}\right) \otimes \Theta_{S \mid C_{x}}^{\vee}\right)=0 .
\end{aligned}
$$

Since $C_{x}$ is smooth, the sheaf $\Theta_{S \mid C_{x}}^{\vee}$ has a filtration with associated graded the direct sum of two copies of $\mathscr{O}_{C_{x}}$. Hence the vector bundle appearing in (6.4.19) has a filtration whose associated graded is the direct sum of two copies of $\operatorname{Sym}^{2} \mathscr{F}_{x}^{\vee} \otimes \bigwedge^{2} \mathscr{F}_{x}$. The latter vector bundle has no nonzero section because it is slope-stable of slope 0 . By the HRR theorem, it follows that

$$
H^{0}\left(C_{x},\left(\operatorname{Sym}^{2} \mathscr{F}_{x}^{\vee} \otimes \bigwedge^{2} \mathscr{F}_{x}\right) \otimes \Theta_{S \mid C_{x}}^{\vee}\right)=H^{1}\left(C_{x},\left(\operatorname{Sym}^{2} \mathscr{F}_{x}^{\vee} \otimes \bigwedge^{2} \mathscr{F}_{x}\right) \otimes \Theta_{S \mid C_{x}}^{\vee}\right)=0 .
$$

Hence the extension group in (6.4.18) vanishes.

The result about traceless endomorphisms of $\mathscr{F}[2]_{\mid \Delta_{x}^{[2]}}^{ \pm}$follows from the direct sum decomposition in (6.4.14) and the vanishing in (6.4.19).

LEMma 6.13. Let $S$ be a $K 3$ surface with an elliptic fibration $S \rightarrow \mathbb{P}^{1}$ as in Claim 6.1, and let $\mathscr{F}$ be a vector bundle on $S$ as in Proposition 6.2. Let $x$ be a regular value of the elliptic fibration. Let $p_{x}: C_{x}^{2} \rightarrow S^{[2]}$ be the composition of the quotient map $C_{x}^{2} \rightarrow C_{x}^{(2)}$ and the inclusion $C_{x}^{(2)} \hookrightarrow S^{[2]}$. Then $p_{x}^{*} \mathscr{F}[2]^{ \pm}$is simple, and hence the restriction of $\mathscr{F}[2]^{ \pm}$to $C_{x}^{(2)}$ is simple.

Proof. The proofs for $\mathscr{F}[2]^{ \pm}$are similar. We give the proof for $\mathscr{F}[2]^{+}$.

Let $q_{x}, r_{x}: C_{x}^{2} \rightarrow C_{x}$ be the two projections, and let $\operatorname{Diag}_{x} \subset C_{x}^{2}$ be the diagonal. By the exact sequence in (5.2.3), we have the exact sequence

$$
0 \longrightarrow p_{x}^{*} \mathscr{F}[2]^{+} \longrightarrow q_{x}^{*} \mathscr{F}_{x} \otimes r_{x}^{*} \mathscr{F}_{x} \stackrel{\mathrm{ev}_{x}^{-}}{\longrightarrow} \bigwedge^{2} \mathscr{F}_{x \mid \operatorname{Diag}_{x}} \longrightarrow 0
$$

It follows that we have the exact sequence

$$
0 \longrightarrow q_{x}^{*} \mathscr{F}_{x} \otimes r_{x}^{*} \mathscr{F}_{x} \otimes \mathscr{O}_{C_{x}^{2}}\left(-\operatorname{Diag}_{x}\right) \stackrel{\lambda}{\longrightarrow} p_{x}^{*} \mathscr{F}[2]^{+} \stackrel{\mathrm{ev}_{x}^{+}}{\longrightarrow} \operatorname{Sym}^{2} \mathscr{F}_{x \mid \operatorname{Diag}_{x}} \longrightarrow 0
$$

Notice that the restriction of $\lambda$ to $\operatorname{Diag}_{x}$ is identified with the natural map ev ${ }_{x}^{-}: \mathscr{F}_{x} \otimes \mathscr{F}_{x} \rightarrow$ $\bigwedge^{2} \mathscr{F}_{x}$ (notice that the normal bundle of $\operatorname{Diag}_{x}$ in $C_{x}^{2}$ is trivial because $C_{x}$ is an elliptic curve); in particular, its image is $\bigwedge^{2} \mathscr{F}_{x}$ (we identify $\operatorname{Diag}_{x}$ and $C_{x}$ ). Equivalently, the restriction of the exact sequence in (6.4.21) to $\operatorname{Diag}_{x}$ gives rise to the exact sequence

$$
0 \longrightarrow \bigwedge^{2} \mathscr{F}_{x} \longrightarrow p_{x}^{*} \mathscr{F}[2]_{\mid \operatorname{Diag}_{x}}^{+} \stackrel{\mathrm{ev}_{x}^{+}}{\longrightarrow} \operatorname{Sym}^{2} \mathscr{F}_{x} \longrightarrow 0
$$




\section{MODUlAR SHEAVES ON HYPERKÄHLER VARIETIES}

which is split by Lemma 6.12 . Now let $\varphi$ be an endomorphism of $p_{x}^{*} \mathscr{F}[2]^{+}$. The restriction of $\varphi$ to $\operatorname{Diag}_{x}$ preserves the exact sequence in (6.4.22) because the vector bundles $\mathrm{Sym}^{2} \mathscr{F}_{x}$ and $\bigwedge^{2} \mathscr{F}_{x}$ are slope-stable of equal slopes. It follows that $\varphi$ induces an endomorphism of the kernel of $\mathrm{ev}_{x}^{+}$, that is, of $q_{x}^{*} \mathscr{F}_{x} \otimes r_{x}^{*} \mathscr{F}_{x} \otimes \mathscr{O}_{C_{x}^{2}}\left(-\operatorname{Diag}_{x}\right)$. Since $\mathscr{F}_{x}$ is simple, the latter is a simple sheaf. It follows that $\varphi$ is a scalar.

Proposition 6.14. Let $S$ be a $K 3$ surface with an elliptic fibration $S \rightarrow \mathbb{P}^{1}$ as in Claim 6.1, and let $\mathscr{F}$ be a vector bundle on $S$ as in Proposition 6.2. Let $x$ be a regular value of the elliptic fibration. Then the restriction of $\mathscr{F}[2]^{ \pm}$to the scheme-theoretic fiber $\pi^{-1}(2 x)$ is a simple sheaf.

Proof. Let $Z_{x}:=\pi^{-1}(2 x)$ be the scheme-theoretic fiber. By Corollary 6.9, we have an exact sequence

$$
0 \longrightarrow \operatorname{End}^{0}(\mathscr{F})_{\mid C_{x}^{(2)}} \otimes \mathscr{O}_{C_{x}^{(2)}}\left(-\Xi_{x}\right) \otimes\left(\mathscr{L}_{\mid C_{x}^{(2)}}\right) \longrightarrow \operatorname{End}^{0}(\mathscr{F})_{\mid Z_{x}} \longrightarrow \operatorname{End}^{0}(\mathscr{F})_{\mid Z_{x}^{\text {red }}} \longrightarrow 0
$$

Taking global sections, we get an isomorphism

$$
H^{0}\left(C_{x}^{(2)}, \operatorname{End}^{0}(\mathscr{F})_{\mid C_{x}^{(2)}} \otimes \mathscr{O}_{C_{x}^{(2)}}\left(-\Xi_{x}\right) \otimes\left(\mathscr{L}_{\mid C_{x}^{(2)}}\right)\right) \stackrel{\sim}{\longrightarrow} H^{0}\left(C_{x}^{(2)}, \operatorname{End}^{0}(\mathscr{F})_{\mid Z_{x}}\right),
$$

because of Lemmas 6.12 and 6.13. On the other hand, since

$$
p_{x}^{*}\left(\mathscr{O}_{C_{x}^{(2)}}\left(-\Xi_{x}\right) \otimes\left(\mathscr{L}_{\mid C_{x}^{(2)}}\right)\right) \cong \mathscr{O}_{C_{x}^{2}}\left(-\operatorname{Diag}_{x}\right)
$$

we have an embedding

$$
H^{0}\left(C_{x}^{(2)}, \operatorname{End}^{0}(\mathscr{F})_{\mid C_{x}^{(2)}} \otimes \mathscr{O}_{C_{x}^{(2)}}\left(-\Xi_{x}\right) \otimes\left(\mathscr{L}_{\mid C_{x}^{(2)}}\right)\right) \longleftrightarrow H^{0}\left(C_{x}^{2}, \operatorname{End}^{0}\left(p_{x}^{*} \mathscr{F}_{x}\right)\left(-\operatorname{Diag}_{x}\right)\right),
$$

and the latter vanishes by Lemma 6.13 .

Proof of Proposition 6.7. Let $B \subset \mathbb{P}^{2}$ be the (finite) set parametrizing $2 x \in\left(\mathbb{P}^{1}\right)^{(2)}$, where $x$ is a critical value of the elliptic fibration $S \rightarrow \mathbb{P}^{1}$. Let us prove that if $\left(x_{1}+x_{2}\right) \in\left(\mathbb{P}^{2} \backslash B\right)$, then

$$
h^{p}\left(\pi^{-1}\left(x_{1}+x_{2}\right), \text { End }^{0} \mathscr{F}[2]_{\mid \pi^{-1}\left(x_{1}+x_{2}\right)}^{ \pm}\right)=0 \quad \forall p .
$$

To see this, first notice that since $\pi^{-1}\left(x_{1}+x_{2}\right)$ is a local complete intersection with trivial dualizing sheaf and $\chi\left(\pi^{-1}\left(x_{1}+x_{2}\right)\right.$, End $\left.^{0} \mathscr{F}[2]_{0 \mid \pi^{-1}\left(x_{1}+x_{2}\right)}^{ \pm}\right)=0$, it suffices to check that (6.4.26) holds for $p=0$.

If $x_{1} \neq x_{2}$, then (6.4.26) holds for $p=0$ by Proposition 6.11. If $x_{1}=x_{2}=x$ and $x$ is a regular value of the elliptic fibration, then (6.4.26) holds for $p=0$ by Proposition 6.14. This proves that $(6.4 .26)$ holds for all $\left(x_{1}+x_{2}\right) \in\left(\mathbb{P}^{2} \backslash B\right)$. Since $B$ is a finite set, we get that

$$
R^{p} \pi_{*} \text { End }^{0} \mathscr{F}[2]^{ \pm}=0, \quad p \in\{0,1\} .
$$

(See [Muk87, Proposition 2.26].) Now suppose that there exist $x_{1}+x_{2} \in \mathbb{P}^{2}$ such that the restriction of $\mathscr{F}[2]^{ \pm}$to $\pi^{-1}\left(x_{1}+x_{2}\right)$ is not simple. As shown above, such points are contained in the finite set $B$, and hence it follows (since the fibers of $\pi$ are surfaces) that $R^{2} \pi_{*} \operatorname{End}^{0}\left(\mathscr{F}[2]^{ \pm}\right)$ is a nonzero Artinian sheaf. By the Leray spectral sequence for $\pi$ and the vanishing in (6.4.27), it follows that $H^{2}\left(S^{[2]}, \operatorname{End}^{0}\left(\mathscr{F}[2]^{ \pm}\right)\right) \neq 0$. This contradicts Proposition 5.4.

\section{Proof of Theorem 1.4 and Corollary 1.7}

7.1. Summary. In Subsection 7.2, we prove that if $d \gg 0$, there exists an irreducible component $\mathscr{N}_{e}^{i}(d)^{\text {good }} \subset \mathscr{N}_{e}^{i}(d)$, where $\mathscr{N}_{e}^{i}(d) \subset \mathscr{K}_{e}^{i}$ is the Lagrangian Noether-Lefschetz divisor defined in Definition B.1, with the following property: if $[(X, h)] \in \mathscr{N}_{e}^{i}(d)^{\text {good }}$ is generic, there exists 


\section{K. G. O'GRADY}

an $h$ slope-stable vector bundle $\mathscr{E}$ on $X$ with $\operatorname{ch}_{0}(\mathscr{E}), \operatorname{ch}_{1}(\mathscr{E}), \operatorname{ch}_{2}(\mathscr{E})$ given by (1.3.4) whose restriction to Lagrangian fibers is slope-stable with the possible exception of a finite set of fibers. We also prove that there exists one component of the relative moduli space of slope-stable vector bundles on polarized HK varieties parametrized by $\mathscr{K}_{e}^{i}$ with $\mathrm{ch}_{0}, \mathrm{ch}_{1}, \mathrm{ch}_{2}$ given by (1.3.4) which dominates the moduli space $\mathscr{K}_{e}^{i}$.

In Subsection 7.3, we prove that if $[(X, h)] \in \mathscr{N}_{e}^{i}(d)^{\text {good }}$ is as above, there is a single $h$ slope-stable vector bundle with the relevant Chern character.

In Subsection 7.4, we prove Theorem 1.4 and Corollary 1.7.

7.2. Good vector bundles over Lagrangian HK varieties. Below is the first main result of the present subsection.

Proposition 7.1. Let $i \in\{1,2\}$, and let $r_{0} \geqslant 2$ be such that $i \equiv r_{0}(\bmod 2)$. Suppose that (1.3.3) holds, that $e \nmid 2 d$ and that

$$
d>\frac{5}{16} r_{0}^{6}\left(r_{0}^{2}-1\right)(e+1) .
$$

There exists an irreducible component $\mathscr{N}_{e}^{i}(d)^{\operatorname{good}} \subset \mathscr{N}_{e}^{i}(d)$, where $\mathscr{N}_{e}^{i}(d) \subset \mathscr{K}_{e}^{i}$ is as in Definition B.1, such that the following holds. Let $[(X, h)] \in \mathscr{N}_{e}^{i}(d)^{\text {good }}$ be generic. (Notice that the hypotheses of Proposition B.2 hold, and hence there is an associated Lagrangian fibration $\pi: X \rightarrow \mathbb{P}^{2}$.) Then

(i) there exists an $h$ slope-stable vector bundle $\mathscr{E}$ on $X$ such that (1.3.4) holds, and

(ii) except possibly for a finite set of $z \in \mathbb{P}^{2}$, the restriction of $\mathscr{E}$ to $\pi^{-1}(z)$ is slope-stable for the restricted polarization.

The proof of Proposition 7.1 is given at the end of the subsection.

Let $S$ be an elliptic $K 3$ surface as in Subsection 6.3, and let us adopt the notation of that subsection. Let $X_{0}=S^{[2]}$, and let $\mathscr{E}_{0}:=\mathscr{F}[2]^{+}$be the vector bundle on $X_{0}$ of loc. cit. Let $h_{0}:=h$, where $h$ is given by (6.3.2). Let $C \subset S$ be a fiber of the elliptic fibration, and let $f_{0}:=\mu(\operatorname{cl}(C))$. Lastly, let $d_{0}$ be as in (6.2.2), and set

$$
d:=i d_{0} .
$$

Then the sublattice $\left\langle f_{0}, h_{0}\right\rangle \subset H_{\mathbb{Z}}^{1,1}\left(X_{0}\right)$ is saturated, and

$$
q\left(f_{0}\right)=0, \quad q\left(h_{0}, f_{0}\right)=d, \quad q\left(h_{0}\right)=e .
$$

Let $\pi_{0}: X_{0} \rightarrow \mathbb{P}^{2}$ be the Lagrangian fibration associated with the elliptic fibration of $S$; see Definition 6.6. Notice that $f_{0}=c_{1}\left(\pi_{0}^{*} \mathscr{O}_{\mathbb{P}^{2}}(1)\right)$.

Let $\varphi: \mathscr{X} \rightarrow B$ be an analytic representative of the deformations space of $\left(X,\left\langle h_{0}, f_{0}\right\rangle\right)$, that is, deformations of $X_{0}$ that keep $h_{0}$ and $f_{0}$ of Hodge type. We assume that $B$ is contractible. Let $0 \in B$ be the base point; in particular, $X_{0}$ is isomorphic to $\varphi^{-1}(0)$. For $b \in B$, we let $X_{b}:=\varphi^{-1}(b)$. If $B$ is small enough, then by Proposition 6.7 and Corollary 5.5 , the vector bundle $\mathscr{E}_{0}$ on $X_{0}$ deforms to a vector bundle $\mathscr{E}_{b}$ on $X_{b}$ (unique up to isomorphism because $\left.H^{1}\left(X_{0}, \operatorname{End}^{0}\left(\mathscr{E}_{0}\right)\right)=0\right)$. Notice that $\left\langle h_{0}, f_{0}\right\rangle$ deforms by Gauss-Manin parallel transport to a saturated sublattice

$$
\Lambda_{b}:=\left\langle h_{b}, f_{b}\right\rangle \subset H_{\mathbb{Z}}^{1,1}\left(X_{b}\right) .
$$

Possibly after shrinking $B$ around 0 , there exists a map $\pi: \mathscr{X} \rightarrow \mathbb{P}^{2}$ which restricts to a Lagrangian fibration on every $X_{b}$ and is equal to $\pi_{0}$ on $X_{0}$. We let $\pi_{b}$ be the restriction of $\pi$ to $X_{b}$. 


\section{MODULAR SHEAVES ON HYPERKÄHLER VARIETIES}

Notice that the fiber of $\varphi \times \pi: \mathscr{X} \rightarrow B \times \mathbb{P}^{2}$ over $(b, z)$ is the Lagrangian fiber of $X_{b} \rightarrow \mathbb{P}^{2}$ over $z$; we denote it by $X_{b, z}$. Of course $f_{b}=c_{1}\left(\pi_{b}^{*} \mathscr{O}_{\mathbb{P}^{2}}(1)\right)$.

Proposition 7.2. With the hypotheses of Proposition 7.1, the following holds. For $b \in B$ outside a proper analytic subset, $h_{b}$ is ample and hence $\left[\left(X_{b}, h_{b}\right)\right] \in \mathscr{N}_{e}^{i}(d)$, where $i \equiv r_{0}(\bmod 2)$. Moreover, $\mathscr{E}_{b}$ is $h_{b}$ slope-stable, and items (i) and (ii) of Proposition 7.1 hold for $\mathscr{E}=\mathscr{E}_{b}$.

Proof. Since $r_{0} \geqslant 2$, inequality (7.2.1) implies that $d>10(e+1)$. Hence the hypotheses of Proposition B. 2 hold. In the proof of that proposition, we show that $h_{b}$ is ample for $b$ outside a proper analytic subset of $B$.

Next we prove that $h_{b}$ is $a\left(\mathscr{E}_{b}\right)$-suitable; see Definition 3.5. We claim that the hypotheses of Proposition 4.1 hold with $a_{0}=a\left(\mathscr{E}_{b}\right)$. This is clear for all the hypotheses, except perhaps for the inequality in (4.1.1). By Proposition 5.2, we have $a\left(\mathscr{E}_{b}\right)=\frac{5}{8} r_{0}^{6}\left(r_{0}^{2}-1\right)$, and hence the inequality in (4.1.1) follows from (7.2.1).

Since $h_{b}$ is $a\left(\mathscr{E}_{b}\right)$-suitable, in order to prove that $\mathscr{E}_{b}$ is $h_{b}$ slope-stable, it suffices to show that the restriction to a generic fiber of the Lagrangian fibration is slope-stable; see Proposition 3.6. This is true for $\mathscr{E}_{0}$ by Proposition 6.11. By the openness of slope-stability, it follows that it is also true for $b \in B$ outside a proper analytic subset.

Next we prove that items (i) and (ii) of Proposition 7.1 hold for $\mathscr{E}=\mathscr{E}_{b}$.

Item (i) holds by Proposition 6.4.

Let us prove that item (ii) holds. First we notice that for $b \in B$ outside a proper closed analytic subset, the restriction of $\mathscr{E}_{b}$ to every Lagrangian fiber is simple. In fact, this holds for $b=0$ by Proposition 6.7, and hence the assertion we made holds by the openness of "simpleness." Let us prove that for $b \in B$ outside a proper closed analytic subset, the restriction of $\mathscr{E}_{b}$ to a smooth Lagrangian fiber is slope-stable. By Proposition 6.11, the restriction of $\mathscr{E}_{0}$ to a generic Lagrangian fiber is slope-stable, and hence the restriction of $\mathscr{E}_{b}$ (for $b \in B$ outside ...) to a generic Lagrangian fiber is slope-stable by the openness of slope-stability. By Proposition A.2, we get that the restriction of $\mathscr{E}_{b}$ (for $b \in B$ outside ... ) to a generic smooth Lagrangian fiber is semi-homogeneous. It follows that the restriction to any smooth Lagrangian fiber is simple semi-homogeneous (note: the fact that the restriction is simple is crucial). By [Muk78, Proposition 6.16], the restriction of $\mathscr{E}_{b}$ to any smooth Lagrangian fiber is Gieseker-Maruyama stable and hence slope-semistable. By Corollary 4.5, it follows that it is actually slope-stable.

Next we claim that the restriction of $\mathscr{E}_{b}$ (for $b \in B$ outside ...) to a generic singular Lagrangian fiber is slope-stable, except possibly for a finite set of fibers. The singular Lagrangian fibers of $X_{b}$ are parametrized by the discriminant curve of $\pi_{b}$, which, for $b \in B$ outside a proper closed analytic subset, is the dual curve of a generic sextic plane curve; see Proposition B.4. On the other hand, the discriminant curve of $\pi_{0}$ is the union of 24 lines (each corresponding to a critical value of the elliptic fibration) and a conic (the "diagonal"). The restriction of $\mathscr{E}_{0}$ to the Lagrangian surface parametrized by a generic point of one of the lines is slope-stable by Proposition 6.11. By the openness of slope-stability, it follows that the locus of singular Lagrangian fibers on which $\mathscr{E}_{b}$ restricts to a slope-stable vector bundle is nonempty (for $b \in B$ outside $\ldots$ ). Since (for $b \in B$ outside ...) the discriminant curve is irreducible, this proves that, with the possible exception of a finite set of singular fibers, the restriction of $\mathscr{E}_{b}$ (for $b \in B$ outside ...) to a generic singular Lagrangian fiber is slope-stable.

Proof of Proposition 7.1. Let $\mathscr{X} \rightarrow T_{e}^{1}$ and $\mathscr{X} \rightarrow T_{e}^{2}$ be complete families of polarized HK varieties of type $K 3^{[2]}$ such that (1.3.1) and (1.3.2), respectively, hold-for example, the families 


\section{K. G. O'GRADY}

parametrized by the relevant open subsets of suitable Hilbert schemes. We may, and will, assume that $T_{e}^{i}$ is irreducible. For $t \in T_{e}^{i}$, we let $\left(X_{t}, h_{t}\right)$ be the corresponding polarized HK variety of type $K 3^{[2]}$. Let $m: T_{e}^{i} \rightarrow \mathscr{K}_{e}^{i}$ be the moduli map, which sends $t$ to $\left[\left(X_{t}, h_{t}\right)\right]$.

By fundamental results of Gieseker and Maruyama, there exists a map of schemes

$$
f: \mathscr{M}_{e}\left(r_{0}\right) \longrightarrow T_{e}^{i}
$$

such that for every $t \in T_{e}^{i}$, the (scheme-theoretic) fiber $f^{-1}(t)$ is isomorphic to the (coarse) moduli space of $h_{t}$ slope-stable vector bundles $\mathscr{E}$ on $X_{t}$ such that (1.3.4) holds. Moreover, $f: \mathscr{M}_{e}\left(r_{0}\right) \rightarrow T_{e}^{i}$ is of finite type by Maruyama [Mar81], and hence $f\left(\mathscr{M}_{e}\left(r_{0}\right)\right)$ is a constructible subset of $T_{e}^{i}$.

By Proposition 7.2, the image of $f: \mathscr{M}_{e}\left(r_{0}\right) \rightarrow T_{e}^{i}$ contains a nonempty subset of $m^{-1}\left(\mathscr{N}_{e}^{i}(d)\right)$ which is open in the classical topology. Since the image of $f$ is a constructible set, it follows that it contains a Zariski-open dense subset of an irreducible component of $m^{-1}\left(\mathscr{N}_{e}^{i}(d)\right)$. Since the image of $f$ is the inverse image of a subset of the moduli map $m$, it follows that there exists an irreducible component $\mathscr{N}_{e}^{i}(d)^{\operatorname{good}} \subset \mathscr{N}_{e}^{i}(d)$ such that the image of $f$ contains a Zariski-open dense subset of $m^{-1}\left(\mathscr{N}_{e}^{i}(d)^{\text {good }}\right)$.

Next we state the second main result of the present subsection. Let us agree that a map of quasi-projective varieties is dominant if the image is Zariski-dense in the codomain (usually, the attribute dominant is reserved to maps between irreducible varieties).

Proposition 7.3. With notation as above, the map $f: \mathscr{M}_{e}\left(r_{0}\right) \rightarrow T_{e}^{i}$ is dominant.

Proof. This follows at once from Corollary 5.5.

7.3. Unicity of stable vector bundles on Lagrangian $\mathbf{H K}$ varieties. We will prove the result below.

Proposition 7.4. Let $i \in\{1,2\}$. Suppose that $r_{0} \geqslant 2$, that $r_{0} \equiv i(\bmod 2)$, that $(1.3 .3)$ holds, that $e \nmid 2 d$ and that

$$
d>\frac{5}{16} r_{0}^{6}\left(r_{0}^{2}-1\right)(e+1) .
$$

Let $[(X, H)] \in \mathscr{N}_{e}^{i}(d)^{\text {good }}$ be generic, where $\mathscr{N}_{e}^{i}(d)^{\text {good }}$ is as in Proposition 7.1. Then, up to isomorphism, there exists one and only one $h$ slope-stable vector bundle $\mathscr{E}$ on $X$ such that (1.3.4) holds.

We first prove the following auxiliary result.

Lemma 7.5. Let $(Y, h)$ be a polarized irreducible smooth projective variety, and let $\rho: Y \rightarrow T$ be a dominant map to a smooth curve with integral fibers of dimension $n$. For $t \in T$, set $Y_{t}:=\rho^{-1}(t)$ and $h_{t}:=h_{\mid Y_{t}}$. Let $\mathscr{F}$ and $\mathscr{G}$ be locally free sheaves on $Y$ such that the following hold:

(i) $\Delta(\mathscr{F}) \smile h^{n-1}=\Delta(\mathscr{G}) \smile h^{n-1}$.

(ii) The restriction $\mathscr{F}_{t}:=\mathscr{F}_{\mid Y_{t}}$ is $h_{t}$ slope-stable for all $t \in T$.

(iii) The restrictions $\mathscr{F}_{t}$ and $\mathscr{G}_{t}:=\mathscr{G}_{\mid Y_{t}}$ are isomorphic for generic $t \in T$.

Then $\mathscr{F}_{t}$ and $\mathscr{G}_{t}$ are isomorphic for all $t \in T$.

Proof. The sheaf $\mathscr{L}:=\operatorname{Hom}_{\rho}(\mathscr{G}, \mathscr{F})$ is torsion-free because $\mathscr{G}$ and $\mathscr{F}$ are locally free, and its fiber over a generic point of $T$ is 1-dimensional by items (ii) and (iii). Since $T$ is a smooth curve, 


\section{MODUlAR SHEAVES ON HYPERKÄHLER VARIETIES}

it follows that $\mathscr{L}:=\operatorname{Hom}_{\rho}(\mathscr{G}, \mathscr{F})$ is an invertible sheaf. The tautological map $\mathscr{G} \otimes \rho^{*}(\mathscr{L}) \rightarrow \mathscr{F}$ gives rise to an exact sequence

$$
0 \longrightarrow \mathscr{G} \otimes \mathscr{L} \stackrel{\alpha}{\longrightarrow} \mathscr{F} \longrightarrow \mathscr{Q} \longrightarrow 0 \text {. }
$$

It suffices to show that $\mathscr{Q}=0$. Suppose that $\mathscr{Q} \neq 0$; we claim that

$$
\int_{Y} \Delta\left(\mathscr{G} \otimes \rho^{*}(\mathscr{L})\right) \smile h^{n-1}>\int_{Y} \Delta(\mathscr{F}) \smile h^{n-1} .
$$

In order to prove this, we notice that the (set-theoretic) support of $\mathscr{Q}$ is a disjoint union of fibers of $\rho$, say $Y_{t_{1}}, \ldots, Y_{t_{d}}$, and that $\alpha_{t}: \mathscr{G}_{t} \rightarrow \mathscr{F}_{t}$ is not an isomorphism if and only if $t \in\left\{t_{1}, \ldots, t_{d}\right\}$. Thus we have $\mathscr{Q}=\oplus_{i=1}^{d} \mathscr{Q}_{i}$, where the set-theoretic support of $\mathscr{Q}_{i}$ is $Y_{t_{i}}$. Let $r=\operatorname{rk}(\mathscr{F})=\operatorname{rk}(\mathscr{G})$. We have

$$
\Delta\left(\mathscr{G} \otimes \rho^{*}(\mathscr{L})\right)=\Delta(\mathscr{F})+2 \sum_{i=1}^{d}\left(r \operatorname{ch}_{2}\left(\mathscr{Q}_{i}\right)-c_{1}(\mathscr{F}) \smile c_{1}\left(\mathscr{Q}_{i}\right)\right) .
$$

Let $\epsilon_{i} \in \mathscr{O}_{T, t_{i}}$ be a local parameter at $t_{i}$. There exist $m_{i}>0$ such that $\epsilon_{i}^{m_{i}} \mathscr{Q}_{i}=0$. For each $t_{i}$, we have a filtration $\mathscr{Q} \supset \epsilon_{i} \cdot \mathscr{Q} \supset \cdots \supset \epsilon_{i}^{m_{i}} \cdot \mathscr{Q}=0$ and

$$
\begin{aligned}
r \operatorname{ch}_{2}\left(\mathscr{Q}_{i}\right)-c_{1}(\mathscr{F}) \smile c_{1}\left(\mathscr{Q}_{i}\right) & \\
\quad= & \sum_{\ell=1}^{m_{i}}\left(r \operatorname{ch}_{2}\left(\epsilon_{i}^{\ell} \cdot \mathscr{Q}_{i} / \epsilon_{i}^{\ell+1} \cdot \mathscr{Q}_{i}\right)-c_{1}(\mathscr{F}) \smile c_{1}\left(\epsilon_{i}^{\ell} \cdot \mathscr{Q}_{i} / \epsilon_{i}^{\ell+1} \cdot \mathscr{Q}_{i}\right)\right) .
\end{aligned}
$$

The sheaf $\epsilon_{i}^{\ell} \cdot \mathscr{Q}_{i} / \epsilon_{i}^{\ell+1} \cdot \mathscr{Q}_{i}$ is annihilated by $\epsilon_{i}$, hence it is the pushforward of a sheaf on $Y_{t_{i}}$ :

$$
\epsilon_{i}^{\ell} \cdot \mathscr{Q}_{i} / \epsilon_{i}^{\ell+1} \cdot \mathscr{Q}_{i}=i_{Y_{i}, *}\left(\overline{\mathscr{Q}}_{i, \ell}\right) \text {. }
$$

By the GRR theorem, we get that

$$
\operatorname{ch}_{2}\left(i_{Y_{t_{i}}, *}\left(\overline{\mathscr{Q}}_{i, \ell}\right)\right)=i_{Y_{t_{i}}, *}\left(c_{1}\left(\overline{\mathscr{Q}}_{i, \ell}\right)\right), \quad c_{1}(\mathscr{F}) \smile c_{1}\left(\overline{\mathscr{Q}}_{i, \ell}\right)=i_{Y_{t_{i}}, *}\left(\operatorname{rk}\left(\overline{\mathscr{Q}}_{i, \ell}\right) i_{Y_{t_{i}}}^{*} c_{1}(\mathscr{F})\right) .
$$

Hence

$$
\begin{gathered}
\int_{Y}\left(r \operatorname{ch}_{2}\left(\epsilon_{i}^{\ell} \cdot \mathscr{Q}_{i} / \epsilon_{i}^{\ell+1} \cdot \mathscr{Q}_{i}\right)-c_{1}(\mathscr{F}) \smile c_{1}\left(\epsilon_{i}^{\ell} \cdot \mathscr{Q}_{i} / \epsilon_{i}^{\ell+1} \cdot \mathscr{Q}_{i}\right)\right) \smile h^{n-1} \\
=\int_{Y_{t_{i}}}\left(r c_{1}\left(\overline{\mathscr{Q}}_{i, \ell}\right)-\operatorname{rk}\left(\overline{\mathscr{Q}}_{i, \ell}\right) c_{1}\left(\mathscr{F}_{t_{i}}\right)\right) \smile h_{t_{i}}^{n-1} .
\end{gathered}
$$

We have surjections

$$
\begin{array}{ccc}
\mathscr{F} / \epsilon_{i} \cdot \mathscr{F} & \stackrel{\phi_{i, \ell}}{\longmapsto} & \epsilon_{i}^{\ell} \cdot \mathscr{Q}_{i} / \epsilon_{i}^{\ell+1} \cdot \mathscr{Q}_{i}, \\
s & \longmapsto & \epsilon_{i}^{\ell} \cdot s .
\end{array}
$$

Notice that we may view $\phi_{i, \ell}$ as a map of sheaves on $Y_{t_{i}}$, namely as $\phi_{i, \ell}: \mathscr{F}_{t_{i}} \rightarrow \overline{\mathscr{Q}}_{i, \ell}$. By the $h_{t_{i}}$ slope-stability of $\mathscr{F}_{t_{i}}$ it follows that if

$$
0<\operatorname{rk}\left(\overline{\mathscr{Q}}_{i, \ell}\right)<r=\operatorname{rk}(\mathscr{F})=\operatorname{rk}(\mathscr{G}),
$$

the integral in (7.3.6) is strictly positive.

Let us prove that (7.3.8) holds if $\ell=0$. The map $\alpha_{t_{i}}: \mathscr{G}_{t_{i}} \rightarrow \mathscr{F}_{t_{i}}$ is nonzero by hypothesis, and $\overline{\mathscr{Q}}_{i, 0}=\operatorname{coker}\left(\alpha_{t_{i}}\right)$. If $\operatorname{rk}_{Y_{t_{i}}}\left(\overline{\mathscr{Q}}_{i, 0}\right)=r$, then $\alpha_{t_{i}}$ vanishes at the generic point of $Y_{t_{i}}$, and hence it vanishes at all points of $Y_{t_{i}}$, which gives a contradiction. Now suppose that $\operatorname{rk}_{Y_{t_{i}}}\left(\overline{\mathscr{Q}}_{i, 0}\right)=0$, that is, that $\alpha_{t_{i}}$ is an isomorphism at the generic point. By item (iii), we have $c_{1}\left(\mathscr{G}_{t_{i}}\right)=c_{1}\left(\mathscr{F}_{t_{i}}\right)$, and it follows that $\alpha_{t_{i}}$ is an isomorphism, which gives a contradiction. We have proved that (7.3.8) holds if $\ell=0$. 


\section{K. G. O'GRADY}

If $\operatorname{rk}\left(\overline{\mathscr{Q}}_{i, 0}\right)=0$, then $c_{1}\left(\overline{\mathscr{Q}}_{i, \ell}\right)$ is effective, and hence the integral in (7.3.6) is positive or zero, and if $\operatorname{rk}\left(\overline{\mathscr{Q}}_{i, 0}\right)=r$, then $\mathscr{F}_{t_{i}} \cong \overline{\mathscr{Q}}_{i, 0}$, and hence the integral in (7.3.6) vanishes.

By (7.3.4) and (7.3.5), we get that (7.3.3) holds.

Since $\Delta\left(\mathscr{G} \otimes \rho^{*}(\mathscr{L})\right)=\Delta(\mathscr{G})$, the inequality in (7.3.3) contradicts item (i).

Proof of Proposition 7.4. The existence has been proved in Proposition 7.1. Let $\mathscr{E}$ be the vector bundle of that proposition. Then $[\mathscr{E}] \in \mathscr{M}_{e}\left(r_{0}\right)$.

Now let $\mathscr{A}$ be any $h$ slope-stable vector such that

$$
\operatorname{ch}_{i}(\mathscr{A})=\operatorname{ch}_{i}(\mathscr{E}) \quad \forall i \in\{0,1,2\}
$$

We must prove that $\mathscr{A}$ is isomorphic to $\mathscr{E}$.

Let $\pi: X \rightarrow \mathbb{P}^{2}$ be the associated Lagrangian fibration of $[(X, h)]$. Since $[(X, h)]$ is a generic point of $\mathscr{N}_{e}^{i}(d)^{\text {good }}$, the discriminant divisor of $\pi$ is the dual of a smooth plane sextic curve (see Proposition B.4), and hence it is smooth away from a finite set $B_{0} \subset \mathbb{P}^{2}$. By item (ii) of Proposition 7.1, there is a finite (possibly empty) set $B_{1} \subset \mathbb{P}^{2}$ of $z$ such that the restriction of $\mathscr{E}$ to $\pi^{-1}(z)$ is not slope-stable.

Let $z_{0} \in\left(\mathbb{P}^{2} \backslash\left(B_{0} \cup B_{1}\right)\right)$. We claim that $\mathscr{A}_{\mid \pi^{-1}\left(z_{0}\right)}$ is isomorphic to $\mathscr{E}_{\mid \pi^{-1}\left(z_{0}\right)}$. In fact, let $T \subset \mathbb{P}^{2}$ be a smooth curve containing $z_{0}$ and intersecting transversely the discriminant divisor of $\pi$. Thus $Y:=\pi^{-1}(T)$ is a smooth threefold, and the restriction of $\pi$ to $Y$ defines a dominant map $\rho: Y \rightarrow T$. We apply Lemma 7.5 to $\mathscr{F}:=\mathscr{E}_{\mid Y}$ and $\mathscr{G}:=\mathscr{A}_{\mid Y}$ (the polarization of $T$ is the restriction of the polarization of $X$ ). Let us check that the hypotheses of that lemma are satisfied: item (i) holds by (7.3.9), item (ii) holds by item (ii) of Proposition 7.1, and item (iii) holds by Proposition 4.1. In fact, by that proposition, the set of $z \in \mathbb{P}^{2}$ such that $\mathscr{E} \mid \pi^{-1}(z)$ is not isomorphic to $\mathscr{A}_{\mid \pi^{-1}(z)}$ is contained in a proper closed subset $Z \subset \mathbb{P}^{2}$, and hence it suffices to choose $T$ so that it is not contained in $Z$. This shows that the hypotheses of Lemma 7.5 hold, and hence we get that the restrictions of $\mathscr{E}$ and $\mathscr{A}$ to any fiber of $Y \rightarrow T$ are isomorphic. In particular, $\mathscr{E}_{\mid \pi^{-1}\left(z_{0}\right)}$ is isomorphic to $\mathscr{A}_{\mid \pi^{-1}\left(z_{0}\right)}$.

Let $z \in\left(\mathbb{P}^{2} \backslash\left(B_{0} \cup B_{1}\right)\right)$. We have proved that $\mathscr{A}_{\mid \pi^{-1}(z)}$ is isomorphic to $\mathscr{E}_{\mid \pi^{-1}(z)}$, and hence in particular $\mathscr{A}_{\mid \pi^{-1}(z)}$ and $\mathscr{E}_{\mid \pi^{-1}(z)}$ are slope-stable. Since $c_{1}(\mathscr{E})=c_{1}(\mathscr{A})$, it follows that the restrictions of $\mathscr{E}$ and $\mathscr{A}$ to $\pi^{-1}\left(\mathbb{P}^{2} \backslash\left(B_{0} \cup B_{1}\right)\right)$ are isomorphic; see the proof of Lemma 7.5. By Hartogs' theorem, it follows that $\mathscr{E}$ is isomorphic to $\mathscr{A}$.

\subsection{Proofs of Theorem 1.4 and Corollary 1.7}

Proof of Theorem 1.4. If $r_{0}=1$, the result is trivially true, hence we may assume that $r_{0} \geqslant 2$. Let $\mathscr{X} \rightarrow T_{e}^{1}$ and $\mathscr{X} \rightarrow T_{e}^{2}$ be complete families of polarized HK varieties of type $K 3^{[2]}$ such that (1.3.1) and (1.3.2), respectively, hold - for example, the families parametrized by the relevant open subsets of suitable Hilbert schemes. Since $\mathscr{K}_{e}^{i}$ is irreducible, we may, and will, assume that $T_{e}^{i}$ is irreducible. By passing to normalization if necessary, we may assume that $T_{e}^{i}$ is normal. For $t \in T_{e}^{i}$, we let $\left(X_{t}, h_{t}\right)$ be the corresponding polarized HK variety of type $K 3^{[2]}$. We let $m: T_{e}^{i} \rightarrow \mathscr{K}_{e}^{i}$ be the moduli map, sending $t$ to $\left[\left(X_{t}, h_{t}\right)\right]$.

Let $f: \mathscr{M}_{e}\left(r_{0}\right) \rightarrow T_{e}^{i}$ be the relative moduli space that we have introduced; see (7.2.5).

By Proposition 7.4, for $t$ in a dense subset of $\bigcup_{d \gg 0} m^{-1}\left(\mathscr{N}_{e}^{i}(d)^{\text {good }}\right)$, the preimage $f^{-1}(t)$ is a singleton. Since $\bigcup_{d \gg 0} m^{-1}\left(\mathscr{N}_{e}^{i}(d)^{\text {good }}\right)$ is Zariski-dense in $T_{e}^{i}$ (it is the union of an infinite collection of pairwise distinct divisors), and since $f\left(\mathscr{M}_{e}\left(r_{0}\right)\right)$ is a constructible subset of $T_{e}^{i}$, it follows that for generic $t \in T_{e}^{i}$, the fiber $f^{-1}(t)$ is a singleton. 


\section{MODUlar SHEAVES ON HYPERKÄHLER VARIETIES}

Let $[\mathscr{E}]$ be the unique point of $f^{-1}(t)$ for $t$ a generic point of $m^{-1}\left(\mathscr{N}_{e}^{i}(d)^{\text {good }}\right)$, where $d \gg 0$. Then $H^{p}\left(X_{t}, \operatorname{End}^{0}(\mathscr{E})\right)=0$ by Proposition 6.7. Hence the last sentence of Theorem 1.4 follows from upper semicontinuity of cohomology.

Proof of Corollary 1.7. Since $T_{e}^{i}$ is normal, it follows by Theorem 1.4 and Zariski's main theorem that every fiber of $f$ is either empty or connected.

Now let $t \in T_{e}^{i}$ be such that $\left(X_{t}, h_{t}\right)$ is isomorphic to $(X, h)$; we identify $X_{t}$ with $X$. Let $x \in f^{-1}(t)$ be the point representing the vector bundle $\mathscr{E}$. Since $h^{2}\left(X, \operatorname{End}^{0}(\mathscr{E})\right)=0$, every irreducible component of $\mathscr{M}_{e}\left(r_{0}\right)$ containing $x$ dominates $T_{e}^{i}$. By Theorem 1.4 , there is a single such component. Hence

$$
\chi\left(X, \operatorname{End}^{0}(\mathscr{E})\right)=\chi\left(X_{s}, \operatorname{End}^{0}(\mathscr{G})\right)
$$

where $s \in T_{e}^{i}$ is generic and $\mathscr{G}$ is the unique (up to isomorphism) $h_{s}$ slope-stable vector bundle on $X_{s}$ such that (1.3.4) holds (with $\mathscr{G}$ replacing $\left.\mathscr{E}\right)$.

By (7.4.1) and Theorem 1.4, we get that $\chi\left(X, \operatorname{End}^{0}(\mathscr{E})\right)=0$. Now $h^{0}\left(X, \operatorname{End}^{0}(\mathscr{E})\right)=0$ by stability, hence $h^{4}\left(X, \operatorname{End}^{0}(\mathscr{E})\right)=0$ by Serre duality, and $h^{2}\left(X, \operatorname{End}^{0}(\mathscr{E})\right)=0$ by hypothesis. It follows that $H^{1}\left(X, \operatorname{End}^{0}(\mathscr{E})\right)=0$ (notice that $H^{1}\left(X, \operatorname{End}^{0}(\mathscr{E})\right)$ is Serre dual to $H^{3}\left(X, \operatorname{End}^{0}(\mathscr{E})\right)$ ), and hence $\{x\}$ is a component of $f^{-1}(t)$. Since $f^{-1}(t)$ is not empty, it is connected, and hence it equals $\{x\}$. This proves Corollary 1.7 .

\section{Moduli of DV varieties}

8.1. Debarre-Voisin vector bundles. Let $X \subset \operatorname{Gr}\left(6, V_{10}\right)$ be a DV variety, and let

$$
0 \longrightarrow \mathscr{S} \longrightarrow \mathscr{O}_{X} \otimes V_{10} \longrightarrow \mathscr{Q} \longrightarrow 0
$$

be the restriction to $X$ of the tautological exact sequence of vector bundles on $\operatorname{Gr}\left(6, V_{10}\right)$. Thus $r(\mathscr{S})=6$ and $r(\mathscr{Q})=4$.

Lemma 8.1. Let $X$ be a $D V$ variety, and let $h \in H_{\mathbb{Z}}^{1,1}(X)$ be the Plücker polarization. Then

$$
\begin{aligned}
\operatorname{ch}_{0}(\mathscr{Q}) & =4, \\
\operatorname{ch}_{1}(\mathscr{Q}) & =h, \\
\operatorname{ch}_{2}(\mathscr{Q}) & =\frac{1}{8}\left(h^{2}-c_{2}(X)\right),
\end{aligned}
$$

where $\eta_{X} \in H^{8}(X ; \mathbb{Z})$ is the fundamental class.

Proof. The first two equations are obvious, and equation (8.1.4) follows from [DV10, Proof of Lemma 4.5, second displayed equation after (12), p. 83] (beware that $c_{i}=c_{i}\left(\mathscr{S}^{\vee}\right)$ ).

Remark 8.2. Let $(X, h)$ be a smooth DV variety. Then $q(h)=22$, and the divisibility of $h$ is 2 , that is, $[(X, h)] \in \mathscr{K}_{22}^{2}$. If one sets $r_{0}=2, i=2$ and $e=22$, then for the vector bundle $\mathscr{E}$, the equations in (1.3.4) give the same rank, $\mathrm{ch}_{1}$ and $\mathrm{ch}_{2}$ as for the quotient vector bundle $\mathscr{Q}$ described above.

Remark 8.3. Let $X$ be the variety of lines in a smooth cubic fourfold in $\mathbb{P}\left(V_{6}\right)$. Let

$$
0 \longrightarrow \mathscr{S} \longrightarrow \mathscr{O}_{X} \otimes V_{6} \longrightarrow \mathscr{Q} \longrightarrow 0
$$

be the restriction to $X$ of the tautological exact sequence of vector bundles on $\operatorname{Gr}\left(2, V_{6}\right)$. Hence the rank of $\mathscr{Q}$ is 4 . Let $h \in H_{\mathbb{Z}}^{1,1}(X)$ be the Plücker polarization. Then (see the equations 


\section{K. G. O'GRADY}

in $(2.1 .1))$ we have

$$
\operatorname{ch}_{0}(\mathscr{Q})=4, \quad \operatorname{ch}_{1}(\mathscr{Q})=h, \quad \operatorname{ch}_{2}(\mathscr{Q})=\frac{1}{8}\left(h^{2}-c_{2}(X)\right) .
$$

Next notice that $q(h)=6$ and the divisibility of $h$ is 2 , that is, $[(X, h)] \in \mathscr{K}_{6}^{2}$. The equations above show that the Chern character of $\mathscr{Q}$ is identified with the Chern character appearing in Theorem 1.4 for $r_{0}=2, i=2$ and $e=6$.

Proposition 8.4. If $X$ is a smooth $D V$ variety with cyclic Picard group, both $\mathscr{Q}$ and $\mathscr{S}$ (see (8.1.1)) are slope-stable vector bundles.

Proof. Let $h \in H_{\mathbb{Z}}^{1,1}(X)$ be the Plücker polarization. Let us prove that $\mathscr{Q}$ is $h$ slope-stable. Suppose that $0 \rightarrow \mathscr{A} \rightarrow \mathscr{Q} \rightarrow \mathscr{B} \rightarrow 0$ is a desemistabilizing sequence. Thus $0<r(\mathscr{A})<4$,

$$
\frac{c_{1}(\mathscr{A}) \cdot h^{3}}{r(\mathscr{A})}=\mu(\mathscr{A}) \geqslant \mu(\mathscr{Q}):=\frac{h^{4}}{4}=363,
$$

and we may assume that $\mathscr{B}$ is torsion-free. By hypothesis, $c_{1}(\mathscr{A})=x h$ for some $x \in \mathbb{Z}$, and hence $x \geqslant 1$. It follows that $c_{1}(\mathscr{B})=(1-x) h$. Since $\mathscr{Q}$ is globally generated, so is $\mathscr{B}$. Thus $x=1$, that is, $c_{1}(\mathscr{B})=0$, and $\mathscr{B}$ is trivial because it is globally generated. Hence $c_{4}(\mathscr{Q})=0$. This gives a contradiction. In fact, following the notation in [DV10, Proof of Lemma 4.5, p. 83], we let $c_{i}=c_{i}\left(\mathscr{S}^{\vee}\right)$. Then (using the formulae in equation (11) of loc. cit.)

$$
c_{4}(\mathscr{Q})=c_{1}^{4}-3 c_{1}^{2} c_{2}+c_{2}^{2}+2 c_{1} c_{3}-c_{4}=9 \eta_{X} .
$$

An analogous proof gives the slope-stability of $\mathscr{S}$.

By the openness of slope-stability, we also get the following result.

Corollary 8.5. If $X$ is a generic $D V$ variety, both $\mathscr{Q}$ and $\mathscr{S}$ are slope-stable vector bundles (for the Plücker polarization).

Remark 8.6. Let $X$ be the variety of lines in a smooth cubic fourfold in $\mathbb{P}\left(V_{6}\right)$, and let $\mathscr{Q}$ be the quotient vector bundle appearing in (8.1.5). If $\operatorname{Pic}(X)$ is cyclic, one can prove that $\mathscr{Q}$ is slope-stable by proceeding as in the proof of Proposition 8.4, except that in the end one does not conclude by a Chern class computation (we have $c_{4}(\mathscr{Q})=0$ ). Rather, one shows directly that there is no trivial quotient $\mathscr{Q} \rightarrow \mathscr{O}_{X}$. In fact, assume that there is such a quotient; then there is a nonzero section of $\mathscr{Q}^{\vee}$, and one gets that the latter is false by considering the dual of the exact sequence in (8.1.5).

Proposition 8.7. If $X$ is a generic $D V$ variety, then the map $V_{10} \rightarrow H^{0}(X, \mathscr{Q})$ induced by (8.1.1) is an isomorphism.

Proof. The vector bundle $\mathscr{S}$ has no global sections because it is slope-stable (by Corollary 8.5) with negative slope. Hence it suffices to prove that $h^{0}(X, \mathscr{Q})=10$. We do this by considering a $K 3$ surface $S$ as in Claim 6.1 with $m_{0}=3$ and large odd $d_{0}$. The vector bundle $\mathscr{F}$ on $S$ of Proposition 6.2 has Mukai vector $v(\mathscr{F})=(2, D, 2)$. We claim that

$$
h^{0}(S, \mathscr{F})=4, \quad h^{1}(S, \mathscr{F})=0, \quad h^{2}(S, \mathscr{F})=0
$$

at least for $d \gg 0$ and "most" $S$. In fact, let $\left(S^{\prime}, D^{\prime}\right)$ be a generic polarized $K 3$ surface with $D^{\prime}$ of square 6 . Then there exists a unique $D^{\prime}$ slope-stable vector bundle $\mathscr{F}^{\prime}$ on $S^{\prime}$ with Mukai vector $\left(2, D^{\prime}, 2\right)$. As is easily checked, $h^{0}\left(S^{\prime}, \mathscr{F}^{\prime}\right)=4$. Since moduli of elliptic $K 3$ surfaces that we are considering are dense in the moduli space of polarized $K 3$ surfaces of degree 6 , we get 


\section{MODUlAR SHEAVES ON HYPERKÄHLER VARIETIES}

that $h^{0}(S, \mathscr{F})=4$ for "most" $S$. By the stability of $\mathscr{F}$, we have $h^{2}(S, \mathscr{F})=0$. Hence the middle equality in (8.1.7) also holds because $\chi(S, \mathscr{F})=4$.

Let $\mathscr{E}_{0}:=\mathscr{F}[2]^{+}$. By definition, there is a canonical isomorphism

$$
H^{0}\left(S^{[2]}, \mathscr{E}_{0}\right) \cong \operatorname{Sym}^{2} H^{0}(S, \mathscr{F}),
$$

and hence $h^{0}\left(S^{[2]}, \mathscr{E}_{0}\right)=10$. From the second equality in $(8.1 .7)$, we also get that $h^{1}\left(S^{[2]}, \mathscr{E}_{0}\right)=0$. Now let $X_{0}:=S^{[2]}$, and let $h_{0}:=h$, where $h$ is given by (6.3.2). Notice that $q\left(h_{0}\right)=22$ and the divisibility of $h_{0}$ is 2 .

Let $\mathscr{X} \rightarrow T$ be an analytic representative of the deformations space of $\left(X_{0}, h_{0}\right)$. Let $t \in T$; by Proposition 6.7 and Corollary 5.5, there is one and only one vector bundle $\mathscr{E}_{t}$ on $X_{t}$ which is a deformation of $\mathscr{E}_{0}$. By Proposition 7.2 , the cohomology class $h_{t}$ is a polarization on $X_{t}$ for $t$ generic in $T$, and $\mathscr{E} t$ is $h_{t}$ slope-stable. But for $t \in T$ generic, $\left(X_{t}, h_{t}\right)$ is isomorphic to a DV variety parametrized by an analytic open subset of $\mathbb{P}\left(\bigwedge^{3} V_{10}^{\vee}\right)$. Hence $\mathscr{E} t$ is isomorphic to the corresponding quotient DV vector bundle $\mathscr{Q}_{t}$ on $X_{t}$ by Theorem 1.4 and Corollary 8.5. Hence $h^{0}\left(X_{t}, \mathscr{E}_{t}\right)=h^{0}\left(X_{0}, \mathscr{E}_{0}\right)=10$ because $h^{1}\left(X_{0}, \mathscr{E}_{0}\right)=0$

\subsection{Proof of Theorem 1.8}

Proof. Let $d$ be the degree of the moduli map

$$
\mathscr{M}_{\text {DV } \rightarrow-\rightarrow} \mathscr{K}_{22}^{2} \text {. }
$$

We have $d \geqslant 1$ because the moduli map is dominant. We need to prove that $d=1$. Let $[(X, h)] \in$ $\mathscr{K}_{22}^{2}$ be a generic point. Then there exist $\left[\sigma_{1}\right], \ldots,\left[\sigma_{d}\right] \in \mathbb{P}\left(\wedge^{3} V_{10}^{\vee}\right)$ such that the corresponding polarized DV varieties $\left(X_{1}, h_{1}\right) \ldots,\left(X_{d}, h_{d}\right)$ are smooth and all isomorphic to $(X, h)$, but the $\operatorname{PGL}\left(V_{10}\right)$-orbits of $\left[\sigma_{1}\right], \ldots,\left[\sigma_{d}\right]$ are pairwise distinct. Let $\mathscr{Q}_{i}$ be the DV quotient vector bundle on $X$ determined by $\sigma_{i}$. By Corollary 8.5, each $\mathscr{Q}_{i}$ is $h$ slope-stable, and hence by Theorem 1.4, all the $\mathscr{Q}_{i}$ are isomorphic to a single vector bundle $\mathscr{E}$. By Proposition 8.7, the surjection $\mathscr{O}_{X_{i}} \otimes V_{10} \rightarrow$ $\mathscr{Q}_{i}$ is identified with the canonical map $\mathscr{O}_{X} \otimes H^{0}(X, \mathscr{E}) \rightarrow \mathscr{E}$. It follows that $d=1$.

Remark 8.8. Let $\left|\mathscr{O}_{\mathbb{P} 5}(3)\right| / / \mathrm{PGL}(6) \rightarrow \mathscr{K}_{6}^{2}$ be the moduli map one gets by associating with a smooth cubic 4 -fold the variety of its lines. This map is birational by Voisin's global Torelli theorem for cubics. Charles [Cha12] inverted the argument: he proved independently that the moduli map $\left|\mathscr{O}_{\mathbb{P} 5}(3)\right| / / \operatorname{PGL}(6) \rightarrow \mathscr{K}_{6}^{2}$ is birational and obtained global Torelli for cubic 4-folds from global Torelli for HK varieties.

Here we notice that Charles' result can also be obtained arguing as in the proof of Theorem 1.8.

8.3 Relation with degenerate DV varieties. In short, one may approach the subject of [DHOV20] from the "opposite" direction. That means starting from $(X, h, \mathscr{E})$, where $X$ is of type $K 3^{[2]}$ (either $S^{[2]}$ for a suitable $K 3$ surface, or a HK variety birational to $S^{[2]}$ ), $h$ is a big and nef class such that $q(h)=22$ and $q\left(h, H^{2}(X ; \mathbb{Z})\right)=(2)$, and $\mathscr{E}$ is a slope-stable vector bundle (or more generally a GM stable torsion-free sheaf) such that (1.3.4) holds with $r_{0}=2$. Then $(X, h)$ should correspond to a degenerate $\sigma \in \bigwedge^{3} V_{10}^{\vee}$ ("degenerate" means that the corresponding Debarre-Voisin variety is not smooth of dimension 4) if $\mathscr{E}$ is not as good as possible, for example $h^{0}(X, \mathscr{E})>10$, or $h^{0}(X, \mathscr{E})=10$ but $\mathscr{E}$ is not globally generated, or $\mathscr{E}$ is globally generated but the corresponding map $X \rightarrow \operatorname{Gr}\left(6, H^{0}(\mathscr{E})\right)$ is not an embedding, or $\mathscr{E}$ is not locally free (one should also take into account the possibility of getting a degenerate $\sigma$ because $h$ is not ample). An example: in the proof of Proposition 8.7, we discussed a case in which $h^{0}(X, \mathscr{E})=10$ 


\section{K. G. O'GRADY}

and $\mathscr{E}$ is globally generated but the corresponding map $X \rightarrow \operatorname{Gr}\left(6, H^{0}(\mathscr{E})\right)$ is not an embedding. The "inverse" approach should allow us to complete the discussion of the family appearing in [DHOV20, Section 8].

\section{Appendix A. Homogeneous and semi-homogeneous vector bundles on abelian varieties}

A.1 Basics. Let $A$ be an abelian variety, and let $A^{\vee}:=\operatorname{Pic}^{0}(A)$ be its dual abelian variety. For $a \in A$, let $T_{a}: A \rightarrow A$ be the translation by $a$. For an invertible sheaf $\xi$ on $A$, we let $[\xi] \in A^{\vee}$ be its isomorphism class.

Definition A.1. A vector bundle $\mathscr{F}$ on $A$ is homogeneous if $T_{a}^{*} \mathscr{F} \cong \mathscr{F}$ for every $a \in A$. It is semi-homogeneous if, for every $a \in A$, there exists a $[\xi] \in \operatorname{Pic}^{0}(A) \operatorname{such}$ that $T_{a}^{*} \mathscr{F} \cong \mathscr{F} \otimes \xi$.

Proposition A.2. Let $(A, \theta)$ be a polarized abelian variety of dimension $n$, and let $\mathscr{F}$ be a $\theta$ slope-stable vector bundle on $A$. If

$$
\int_{A} \Delta(\mathscr{F}) \smile \theta^{n-2}=0
$$

(the condition is to be understood to be empty if $n=1$ ), then $\mathscr{F}$ is simple semi-homogeneous. Moreover, $\Delta(\mathscr{F})=0$.

Proof. Of course $\mathscr{F}$ is simple because it is slope-stable.

If $n=1$, then $\mathscr{F}$ is semi-homogeneous by Atiyah's classification of simple vector bundles on elliptic curves.

Suppose that $n \geqslant 2$. By the Kobayashi-Hitchin correspondence, $\mathscr{F}$ has a $\theta$ HermitianEinstein metric, and hence so does the vector bundle $\operatorname{End}(\mathscr{F})$. Equation (A.1.1) is equivalent to $\int_{A} c_{2}(\operatorname{End}(\mathscr{F})) \smile \theta^{n-2}=0$. Since $c_{1}(\operatorname{End}(\mathscr{F}))=0$, the Hermite-Einstein connection on $\operatorname{End}(\mathscr{F})$ is flat; see [Kob82, Section 4]. Hence $\operatorname{End}(\mathscr{F})$ is homogeneous, and thus $\mathscr{F}$ is semi-homogeneous by [Muk78, Theorem 5.8].

Since $\operatorname{End}(\mathscr{F})$ is homogeneous, it is a direct sum of vector bundles which have filtrations whose associated graded bundles are direct sums of a topologically trivial line bundles; see [Muk78, Theorem 4.17]. Thus $\Delta(\mathscr{F})=c_{2}(\operatorname{End}(\mathscr{F}))=0$.

A.2 Rank of semi-homogeneous vector bundles. The rank of a simple semi-homogeneous vector bundle with assigned first Chern class is not arbitrary. Below we extend a result of Mukai, see [Muk78, Theorem 7.11 and Remark 7.13], so that we cover all the canonical polarizations that occur on Lagrangian fibrations in the known deformation classes of HK varieties with the exception of OG10.

Proposition A.3 (Mukai [Muk78]). Let $(A, \theta)$ be a polarized abelian variety of dimension $n$. Suppose that the elementary divisors of $\theta$ are $\left(1, \ldots, 1, d_{1}, d_{2}\right)$, where $d_{1}$ divides $d_{2}$. Let $\mathscr{F}$ be a simple semi-homogeneous vector bundle on $A$ such that $c_{1}(\mathscr{F})=a \theta$. Then there exists a positive integer $r_{0}$ such that, letting $g_{i}:=\operatorname{gcd}\left\{r_{0}, d_{i}\right\}$, we have

$$
\operatorname{gcd}\{r(\mathscr{F}), a\}=\frac{r_{0}^{n-1}}{g_{1} \cdot g_{2}}, \quad r(\mathscr{F})=\frac{r_{0}^{n}}{g_{1} \cdot g_{2}} .
$$

Proof. Let $r:=r(\mathscr{F})$, and let $c:=\operatorname{gcd}\{r, a\}$. We let $r_{0}:=r / c$ and $a_{0}:=a / c$. Let us prove that (A.2.1) holds. Let $\mathscr{L}$ be a line bundle on $A$ such that $c_{1}(\mathscr{L})=a_{0} \theta$, and let $\varphi \mathscr{L}: A \rightarrow A^{\vee}$ be 


\section{MODULAR SHEAVES ON HYPERKÄHLER VARIETIES}

the homomorphism defined by $\varphi \mathscr{L}(a):=T_{a}^{*}(\mathscr{L}) \otimes \mathscr{L}^{-1}$. Let $K(\mathscr{L})$ be the kernel of $\varphi \mathscr{L}$. Lastly, following Mukai, we let

$$
\Sigma(\mathscr{F}):=\left\{[\xi] \in A^{\vee} \mid \exists \stackrel{\sim}{\longrightarrow} \otimes \xi\right\} .
$$

(Since we are in characteristic 0 , the above set-theoretic definition coincides with the schematic one; see [Muk78, Proposition 5.9].) By [Muk78, Corollary 7.8], we have an exact sequence of groups

$$
0 \longrightarrow A\left[r_{0}\right] \cap K(\mathscr{L}) \longrightarrow A\left[r_{0}\right] \stackrel{\varphi_{\mathscr{L}}}{\longrightarrow} \Sigma(\mathscr{F}) \longrightarrow 0 .
$$

Because of our hypothesis on the elementary divisors of $\theta$, we have

$$
K(\mathscr{L}) \cong\left(\mathbb{Z} /\left(a_{0}\right)\right)^{2} \oplus \cdots \oplus\left(\mathbb{Z} /\left(a_{0} d_{1}\right)\right)^{2} \oplus\left(\mathbb{Z} /\left(a_{0} d_{2}\right)\right)^{2} .
$$

Since $a_{0}$ and $r_{0}$ are coprime, it follows that $A\left[r_{0}\right] \cap K(\mathscr{L}) \cong\left(\mathbb{Z} /\left(g_{1}\right)\right)^{2} \oplus\left(\mathbb{Z} /\left(g_{2}\right)\right)^{2}$. Thus $|\Sigma(\mathscr{F})|=r_{0}^{2 n} /\left(g_{1}^{2} \cdot g_{2}^{2}\right)$. On the other hand, the cardinality of $\Sigma(\mathscr{F})$ is equal to $r^{2}$ by [Muk78, Proposition 7.1]. Thus we get that $r=r_{0}^{n} /\left(g_{1} \cdot g_{2}\right)$. Since $c:=r / r_{0}$, it follows that $\operatorname{gcd}\{r, a\}=$ $c=r_{0}^{n-1} /\left(g_{1} \cdot g_{2}\right)$.

\section{Appendix B. Polarized Lagrangian HK varieties of type $K 3^{[2]}$}

B.1 Lagrangian Noether-Lefschetz loci. We recall that $\mathscr{K}_{e}^{i}$ is the moduli space of polarized HK varieties of type $K 3^{[2]}$ with polarization of BBF square $e$ and divisibility given by $i$ (which is either 1 or 2); see Subsection 1.3.

Definition B.1. For $d$ a strictly positive integer, let $\mathscr{N}_{e}^{i}(d) \subset \mathscr{K}_{e}^{i}$ be the closure of the locus parametrizing polarized $\mathrm{HK}$ varieties $(X, h)$ such that $H_{\mathbb{Z}}^{1,1}(X)$ contains a saturated rank 2 sublattice generated by $h$ and $f$, where

$$
q(f)=0, \quad q(h, f)=d .
$$

Proposition B.2. Keeping the notation as above, suppose in addition that $d$ is even if $i=2$ and that

$$
d>5(e+1), \quad e \nmid 2 d .
$$

Then $\mathscr{N}_{e}^{i}(d)$ is closed of pure codimension 1 (in particular, nonempty), and if $[(X, h)] \in \mathscr{N}_{e}^{i}(d)$ is generic, there is one and only one Lagrangian fibration $\pi: X \rightarrow \mathbb{P}^{2}$ (modulo automorphisms of $\left.\mathbb{P}^{2}\right)$ such that, letting $f:=c_{1}\left(\pi^{*} \mathscr{O}_{\mathbb{P}^{2}}(1)\right)$, the equalities in (B.1.1) hold and the sublattice $\langle h, f\rangle \subset H_{\mathbb{Z}}^{1,1}(X)$ is saturated.

Proof. Before starting the proof, we emphasize that $\mathscr{N}_{e}^{i}(d)$ might have several irreducible components and that "generic point" of $\mathscr{N}_{e}^{i}(d)$ means point belonging to an open dense subset of $\mathscr{N}_{e}^{i}(d)$. By the surjectivity of the period map, there exists a HK variety $X$ of type $K 3^{[2]}$ such that $H_{\mathbb{Z}}^{1,1}(X)=\langle h, f\rangle$, where

$$
q(h)=e, \quad\left\{q(h, \alpha) \mid \alpha \in H^{2}(X ; \mathbb{Z})\right\}=(i),
$$

and the equalities in (B.1.1) hold. There are no $\xi \in H_{\mathbb{Z}}^{1,1}(X)$ with $-10 \leqslant q(\xi)<0$ by Lemma 4.3 and the inequality in (B.1.2). It follows that the ample cone of $X$ is equal to the intersection of $H_{\mathbb{Z}}^{1,1}(X)$ and the positive cone. Hence either $h$ or $-h$ is ample. If the former holds, then $[(X, h)] \in \mathscr{N}_{e}^{i}(d)$; if the latter holds, we may replace $h$ and $f$ with $-h$ and $-f$, respectively, and again we get that $[(X, h)] \in \mathscr{N}_{e}^{i}(d)$. Moreover, $\mathscr{N}_{e}^{i}(d)$ is closed of pure codimension 1 because it is a Noether-Lefschetz divisor. 


\section{K. G. O'GRADY}

A straightforward computation shows that there are exactly two primitive nef isotropic classes, namely $f$ and $\alpha:=(1 / \operatorname{gcd}\{d, e\})(2 d h-e f)$. By our "nondivisibility" hypothesis in (B.1.2), we get that $q(\alpha, h)=d e / \operatorname{gcd}\{d, e\}$ is not equal to $d$. Hence $f$ is the unique primitive nef isotropic class such that $q(h, f)=d$.

By [Mar14, Theorem 1.3] (see also [Mar14, Remark 1.8]), there exists a Lagrangian fibration $\pi: X \rightarrow \mathbb{P}^{2}$ such that $f:=c_{1}\left(\pi^{*} \mathscr{O}_{\mathbb{P}^{2}}(1)\right)$. By [Mat17, Theorem 1.2], it follows that there exists a Zariski-open neighborhood $\mathscr{U}$ of $[(X, h)]$ in $\mathscr{N}_{e}^{i}(d)$ such that each representative $\left(X^{\prime}, h^{\prime}\right)$ of a point in $\mathscr{U}$ has a Lagrangian fibration as required. Since the set of points of $\mathscr{N}_{e}^{i}(d)$ representing $(X, h)$ such that $\rho(X)=2$ is dense, this proves the result about the existence of the required Lagrangian fibration.

It remains to prove that if $[(X, h)] \in \mathscr{N}_{e}^{i}(d)$ is generic, then there is a unique isotropic class $f$ such that $q(h, f)=d$. We checked that this is the case if $\rho(X)=2$. It follows that the statement holds for the generic point of $\mathscr{N}_{e}^{i}(d)$; the argument is similar to that given to show that $h$ is $a(\mathscr{E})$-suitable in the proof of Proposition 4.1.

Definition B.3. Suppose that (B.1.2) holds. We let $\mathscr{N}_{e}^{i}(d)^{0} \subset \mathscr{N}_{e}^{i}(d)$ be an open dense subset such that the thesis of Proposition B.2 holds for any $[(X, h)] \in \mathscr{N}_{e}^{i}(d)^{0}$. For $[(X, h)] \in \mathscr{N}_{e}^{i}(d)^{0}$, the associated Lagrangian fibration $\pi: X \rightarrow \mathbb{P}^{2}$ is the unique fibration (modulo automorphisms of $\mathbb{P}^{2}$ ) of Proposition B.2.

B.2 Tate-Shafarevich twists. A basic example of Lagrangian fibration is constructed as follows. Let $S \rightarrow \mathbb{P}^{2}$ be the double cover ramified over a smooth sextic curve $B$, that is, a polarized $K 3$ surface of degree 2 . Let $\mathscr{J}(S)$ be the moduli space of rank 0 pure $\mathscr{O}_{S}(1)$ semistable sheaves $\xi$ with $\chi(\xi)=-1$. The generic point of $\mathscr{J}(S)$ is represented by $i_{*} \mathscr{L}$, where $i: C \hookrightarrow S$ is the inclusion of a smooth $C \in \mathscr{O}_{S}(1)$ and $\mathscr{L}$ is a line bundle of degree 0 . Then for generic $B$, every semistable sheaf is stable (the precise condition is that $B$ have no tritangents), and hence $\mathscr{J}(S)$ is smooth. In fact, it is a HK variety of type $K 3^{[2]}$, and the support map $\mathscr{J}(S) \rightarrow\left(\mathbb{P}^{2}\right)^{\vee}$ is a Lagrangian fibration.

A Lagrangian fibration parametrized by a generic point of $\mathscr{N}_{e}^{i}(d)$ is related to a generic $\mathscr{J}(S)$ via a Tate-Shafarevich twist. In order to be more precise, we recall a result of Markman. Let $(X, h)$ be a representative of a generic point of $\mathscr{N}_{e}^{i}(d)$. Then there is an associated polarized $K 3$ surface $(S, D)$ of degree 2, and moreover $(S, D)$ is generic; see [Mar14, Subsection 4.1].

Proposition B.4. Keep the hypotheses of Proposition B.2. Let $[(X, h)]$ be a generic point of $\mathscr{N}_{e}^{i}(d)$. Let $\pi: X \rightarrow \mathbb{P}^{2}$ and $S$ be the associated Lagrangian fibration and $K 3$ surface of degree 2 , respectively. Then $X$ is isomorphic to a Tate-Shafarevich twist of $\mathscr{J}(S) \rightarrow\left(\mathbb{P}^{2}\right)^{\vee}$ via an identification $\mathbb{P}^{2} \stackrel{\sim}{\rightarrow}\left(\mathbb{P}^{2}\right)^{\vee}$.

Proof. First suppose that $\rho(X)=2$. Then, as shown in the proof of Proposition B.2, the ample cone of $X$ is equal to the positive cone (because of the inequality in (B.1.2)), and hence every bimeromorphic map $X \rightarrow X^{\prime}$, where $X^{\prime}$ is a $H K$ variety, is actually an isomorphism. It follows that $X$ is isomorphic to a Tate-Shafarevich twist of $\mathscr{J}(S) \rightarrow\left(\mathbb{P}^{2}\right)^{\vee}$ by [Mar14, Theorem 7.13]. The result follows from this because the locus in $\mathscr{N}_{e}^{i}(d)$ parametrizing the $(X, h)$ such that $\rho(X)=2$ is dense.

Let $X \rightarrow \mathbb{P}^{2}$ be as in Proposition B.4, and let $\operatorname{Pic}^{0}\left(X / \mathbb{P}^{2}\right)$ be the relative Picard scheme (notice that all fibers of $X \rightarrow \mathbb{P}^{2}$ are irreducible by Proposition B.4). If $U \subset \mathbb{P}^{2}$ is the open dense set of regular values of $X \rightarrow \mathbb{P}^{2}$ and $z \in U$, the fiber of $\operatorname{Pic}^{0}\left(X / \mathbb{P}^{2}\right) \rightarrow \mathbb{P}^{2}$ over $z$ is an abelian 


\section{MODUlAR SHEAVES ON HYPERKÄHLER VARIETIES}

surface $A_{z}$ and the fundamental group $\pi_{1}(U, z)$ acts by monodromy on the subgroup $A_{z \text {,tors }}$ of torsion points.

Corollary B.5. Keep the hypotheses as above, and suppose that $V \subset A_{z}\left[r_{0}^{2}\right]$ is a coset (of a subgroup) of cardinality $r_{0}^{4}$ invariant under the action of monodromy. Then $V=A_{z}\left[r_{0}\right]$.

Proof. Let $S$ be the polarized $K 3$ surface of degree 2 associated with $X$ following Markman, and let $S \rightarrow \mathbb{P}^{2}$ be the double cover ramified over a sextic curve $B$. Let $\mathscr{J}(S)_{0} \subset \mathscr{J}(S)$ be the open dense subset of smooth points (that is, smooth points of $\mathscr{J}(S)$ with surjective differential) of the map $\mathscr{J}(S) \rightarrow\left(\mathbb{P}^{2}\right)^{\vee}$. By Proposition B.4, the relative Picard scheme $\operatorname{Pic}^{0}\left(X / \mathbb{P}^{2}\right) \rightarrow \mathbb{P}^{2}$ is isomorphic to $\mathscr{J}(S)_{0} \rightarrow\left(\mathbb{P}^{2}\right)^{\vee}$ for a certain identification $\mathbb{P}^{2} \stackrel{\sim}{\rightarrow}\left(\mathbb{P}^{2}\right)^{\vee}$. Under this identification, $z \in \mathbb{P}^{2}$ corresponds to a line $R \in\left(\mathbb{P}^{2}\right)^{\vee}$ transverse to $B$, and the corresponding Lagrangian fiber $A_{z}$ is the Jacobian of the curve $C$ which is the double cover of $R$ ramified over $R \cap B$. Hence we have a natural isomorphism

$$
H_{1}(C ; \mathbb{Q}) / H_{1}(C ; \mathbb{Z}) \stackrel{\sim}{\longrightarrow} A_{z, \text { tors }},
$$

and the identification is compatible with the monodromy actions.

First we prove the result under the assumption that $V$ is a subgroup $G$. By the structure theorem for finite abelian groups, $G \cong \mathbb{Z} /\left(d_{1}\right) \oplus \cdots \oplus \mathbb{Z} /\left(d_{r}\right)$, where $r \leqslant 4$ (because $A_{z}\left[r_{0}^{2}\right] \cong \mathbb{Z} /\left(r_{0}^{2}\right)^{\oplus 4}$ ) and $d_{i} \mid r_{0}^{2}$ for all $i$. Since the monodromy action on $H_{1}(C ; \mathbb{Z})$ is transitive on nonzero elements, it follows from the isomorphism in (B.2.1) that $r=4$ and $d_{1}=\cdots=d_{r}$. Thus $d_{i}=r_{0}$ for all $i \in\{1, \ldots, 4\}$ because $|G|=r_{0}^{4}$. This proves the result under the assumption that $V$ is a subgroup.

Now let $V$ be a translate of a group $G$. Then $G=\{a-b \mid a, b \in V\}$, and hence $G$ is also invariant for the monodromy action. Thus $G=A_{z}\left[r_{0}\right]$, and hence the coset $V$ gives a point of the quotient $A_{z}\left[r_{0}^{2}\right] / A_{z}\left[r_{0}\right]$ which is invariant for the monodromy action. By the isomorphism in (B.2.1), it follows that 0 is the unique invariant element, and hence $V=A_{z}\left[r_{0}\right]$.

\section{ACKNOWLEDGEMEnTS}

Many thanks go to the (anonymous) referee for their numerous suggestions. In particular, I owe to the referee the proof of Proposition 5.4 via the MacKay correspondence (the original proof was done by brute force).

\section{REFERENCES}

BD85 A. Beauville and R. Donagi, La variété des droites d'une hypersurface cubique de dimension 4, C. R. Acad. Sci. Paris Sér. I Math. 301 (1985), no. 14, 703-706.

Bea07 A. Beauville, Riemannian holonomy and algebraic geometry, Enseign. Math. 53 (2007), no. 1-2, 97-126.

Bog95 F. A. Bogomolov, Stable vector bundles on projective surfaces, Sb. Math. 81 (1995), no. 2, 397-419; doi:10.1070/SM1995v081n02ABEH003544.

Bog96_ On the cohomology ring of a simple hyper-Kähler manifold (on the results of Verbitsky), Geom. Funct. Anal. 6 (1996), no. 4, 612-618; doi:10.1007/BF02247113.

Cha12 F. Charles, A remark on the Torelli theorem for cubic fourfolds, 2012, arXiv:1209.4509.

DHOV20 O. Debarre, F. Han, K. O'Grady and C. Voisin, Hilbert squares of K3 surfaces and DebarreVoisin varieties, J. Éc. polytech. Math. 7 (2020), 653-710; doi:10.5802/jep.125.

DV10 O. Debarre and C. Voisin, Hyper-Kähler fourfolds and Grassmann geometry, J. reine angew. Math. 649 (2010), 63-87; doi:10.1515/CRELLE.2010.089. 


\section{K. G. O'GRADY}

Fuj87 A. Fujiki, On the de Rham cohomology group of a compact Kähler symplectic manifold, Algebraic Geometry (Sendai, 1985), Adv. Stud. Pure Math., vol. 10 (North-Holland, Amsterdam, 1987), 105-165; doi:10.2969/aspm/01010105.

GRT19 D. Greb, J. Ross and M. Toma, A master space for moduli spaces of Gieseker-stable sheaves, Transform. Groups 24 (2019), no. 2, 379-401; doi:10.1007/s00031-018-9477-6.

Hai01 M. Haiman, Hilbert schemes, polygraphs and the Macdonald positivity conjecture, J. Amer. Math. Soc. 14 (2001), no. 4, 941-1006; doi:10.1090/S0894-0347-01-00373-3.

HL10 D. Huybrechts and M. Lehn, The geometry of moduli spaces of sheaves, 2nd edn, Cambridge Math. Lib. (Cambridge Univ. Press, Cambridge, 2010); doi:10.1017/CB09780511711985.

IM19 D. Iacono and M. Manetti, On deformations of pairs (manifold, coherent sheaf), Canad. J. Math. 71 (2019), no. 5, 1209-1241; doi:10.4153/cjm-2018-027-8.

Kob82 S. Kobayashi, Curvature and stability of vector bundles, Proc. Japan Acad. Ser. A Math. Sci. 58 (1982), no. 4, 158-162; doi:10.3792/pjaa.58.158.

Kru18 A. Krug, Remarks on the derived McKay correspondence for Hilbert schemes of points and tautological bundles, Math. Ann. 371 (2018), no. 1-2, 461-486; doi:10.1007/s00208-0181660-5.

Kul90 S.A. Kuleshov, Exceptional bundles on K3 surfaces, in Helices and Vector Bundles, London Math. Soc. Lecture Note Ser., vol. 148 (Cambridge Univ. Press, Cambridge, 1990), 105-114; doi:10.1017/CB09780511721526.010.

Mar14 E. Markman, Lagrangian fibrations of holomorphic-symplectic varieties of $K 3^{[n]}$-type, in Algebraic and Complex Geometry, Springer Proc. Math. Stat., vol. 71 (Springer, Cham, 2014), 241-283; doi:10.1007/978-3-319-05404-9_10.

Mar78 M. Maruyama, Moduli of stable sheaves. II, J. Math. Kyoto Univ. 18 (1978), no. 3, 557-614; doi: 10.1215/kjm/1250522511.

Mar81_ On boundedness of families of torsion free sheaves, J. Math. Kyoto Univ. 21 (1981), no. 4, 673-701; doi:10.1215/kjm/1250521908.

Mat17 D. Matsushita, On isotropic divisors on irreducible symplectic manifolds, in Higher Dimensional Algebraic geometry - in Honour of Professor Yujiro Kawamata's Sixtieth Birthday, Adv. Stud. Pure Math., vol. 74 (Math. Soc. Japan, Tokyo, 2017), 291-312; doi:10.2969/aspm/07410291.

MR21 G. Mongardi and A. Rapagnetta, Monodromy and birational geometry of O'Grady's sixfolds, J. Math. Pures Appl. 146 (2021), 31-68; doi:10.1016/j.matpur.2020.12.006.

Muk78 S. Mukai, Semi-homogeneous vector bundles on an Abelian variety, J. Math. Kyoto Univ. 18 (1978), no. 2, 239-272; doi:10.1215/kjm/1250522574.

Muk87_ On the moduli space of bundles on K3 surfaces. I, Vector Bundles on Algebraic Varieties (Bombay, 1984), Tata Inst. Fund. Res. Stud. Math., vol. 11 (Tata Inst. Fund. Res., Bombay, 1987), 341-413.

O'G97 K. G. O'Grady, The weight-two Hodge structure of moduli spaces of sheaves on a K3 surface, J. Algebraic Geom. 6 (1997), no. 4, 599-644.

Ver96a M. Verbitsky, Cohomology of compact hyper-Kähler manifolds and its applications, Geom. Funct. Anal. 6 (1996), no. 4, 601-611; doi:10.1007/BF02247112.

Ver96b_ Hyperholomorphic bundles over a hyper-Kähler manifold, J. Algebraic Geom. 5 (1996), no. 4, 633-669.

Wie16 B. Wieneck, On polarization types of Lagrangian fibrations, Manuscripta Math. 151 (2016), no. 3-4, 305-327; doi:10.1007/s00229-016-0845-z.

Wie18 Monodromy invariants and polarization types of generalized Kummer fibrations, Math. Z. 290 (2018), no. 1-2, 347-378; doi:10.1007/s00209-017-2020-y.

Kieran G. O'Grady ogrady@mat.uniroma1.it

Dipartimento di Matematica, Sapienza Università di Roma, P.le A. Moro 5, 00185 Roma, Italy 